\title{
Enantioselective Addition of Activated Terminal Alkynes to 1-Acylpyridinium Salts Catalyzed by Cu-Bis(oxazoline) Complexes
}

\author{
Zhankui Sun, Shouyun Yu, Zuoding Ding and Dawei Ma* \\ State Key Laboratory of Bioorganic and Natural Products Chemistry, \\ Shanghai Institute of Organic Chemistry, Chinese Academy of Sciences, \\ 354 Fenglin Lu, Shanghai 200032, China
}

\section{Supporting Information}

\section{Table of contents}

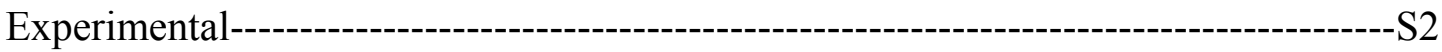

Copies of ${ }^{1} \mathrm{H}$ NMR and ${ }^{13} \mathrm{C}$ NMR spectra of compounds 3a-3h, and 6-10-----------S15 


\section{Experimental}

General methods: All the reactions were carried out under an argon atmosphere with dry solvents under anhydrous conditions unless otherwise indicated. Optical rotations were measured by used a Perkin-Elmer $241 \mathrm{MC}$ polarimeter in the solvent indicated. IR spectra were recorded on an AVATAR-360 spectrophotometer. ${ }^{1} \mathrm{H}$ and ${ }^{13} \mathrm{C}$ NMR spectra were recorded on MERCURY300, Bruker DRX-400, and Bruker AV-500 spectrometers with TMS as the internal standard. HRMS were recorded by using either FTMS-7 or IonSpec 4.7 spectrometers. Silica gel plate GF254 were used for thin layer chromatography (TLC) and silica gel H or 300-400 mesh were used for flash column chromatography. Yields refer to chromatographically and spectroscopically pure compounds, unless otherwise indicated. Enantiomeric excesses were determined by HPLC using a Daicel Chiralpak $\mathrm{AD}-\mathrm{H}$ or OD-H column (wavelength $=214,220$ or $230 \mathrm{~nm}$ ) with hexane/i-PrOH as the eluent.

Material. All solvents and CuI were purified according to Purification of Laboratory Chemicals (4th edition), W. L. F. Armarego \& D. D. Perrin, Butterworth Heinemann.

General procedure for addition of alkynes to 1-acylpyridium salts: $\mathrm{CuI}$ (10 $\mathrm{mg}, 0.05 \mathrm{mmol})$ and ligand $\mathbf{L 5}(20 \mathrm{mg}, 0.05 \mathrm{mmol})$ were dissolved in $1 \mathrm{~mL}$ of $\mathrm{CH}_{2} \mathrm{Cl}_{2}$ and the solution was cooled to $-78{ }^{\circ} \mathrm{C}$. 1-Alkyne $(2.5 \mathrm{mmol})$ and DIPPA (0.6 $\mathrm{mL}, 2.5 \mathrm{mmol}$ ) were then added, and the solution was stirred at this temperature for $30 \mathrm{~min}$ before it was transferred to a flask containing the 1-acylpyridinium salt (generated from $0.5 \mathrm{mmol}$ of pyridine and $2.5 \mathrm{mmol}$ of methyl chloroformate) at -78 
${ }^{\circ} \mathrm{C}$ by a syringe. The resultant mixture was stirred for $15 \mathrm{~h}$ at the same temperature and then was directly purified by chromatography to afford the addition products.

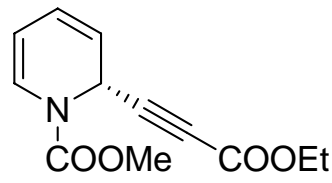

$3 a$

(R)-Methyl 2-(2-(ethoxycarbonyl)ethynyl)pyridine-1(2H)-carboxylate (3a): $72 \%$ yield. $[\alpha]_{\mathrm{D}}{ }^{29}+683\left(\mathrm{c} 1.1, \mathrm{CHCl}_{3}\right) ;{ }^{1} \mathrm{H} \mathrm{NMR}\left(300 \mathrm{MHz}, \mathrm{CDCl}_{3}\right) \delta 6.78 \& 6.67$ (due to rotamers, both d, $J=7.8 \mathrm{~Hz}, 1 \mathrm{H}), 6.02(\mathrm{~m}, 1 \mathrm{H}), 5.75 \& 5.62$ (due to rotamers, both d, $J=6.3 \mathrm{~Hz}, 1 \mathrm{H}), 5.50(\mathrm{~m}, 1 \mathrm{H}), 5.34(\mathrm{~m}, 1 \mathrm{H}), 4.18(\mathrm{q}, J=7.5 \mathrm{~Hz}, 2 \mathrm{H}), 3.8$ (s, 3H), $1.26(\mathrm{t}, J=6.6 \mathrm{~Hz}, 3 \mathrm{H}) ;{ }^{13} \mathrm{C} \mathrm{NMR}\left(75 \mathrm{MHz}, \mathrm{CDCl}_{3}\right) \delta 153.3,153.2,124.6$, 123.6, 116.2, 105.4, 82.0, 75.0, 62.1, 53.7, 43.6, 13.9; MS (ESI) $\mathrm{m} / \mathrm{z} 258.1(\mathrm{M}+\mathrm{Na})^{+}$; HRMS $m / z$ calcd for $\mathrm{C}_{12} \mathrm{H}_{13} \mathrm{NO}_{4} \mathrm{Na}(\mathrm{M}+\mathrm{Na})^{+}$258.0737, found 258.0737; HPLC DAICEL CHIRALPAK AD-H, hexane/2-proponal $=80 / 20$, flow rate: $0.8 \mathrm{~mL} / \mathrm{min}$, detector: $214 \mathrm{~nm}, \mathrm{t}_{\mathrm{R}}($ minor $)=8.22 \mathrm{~min}, \mathrm{t}_{\mathrm{R}}($ major $)=9.70 \mathrm{~min}$, ee $=94 \%$.

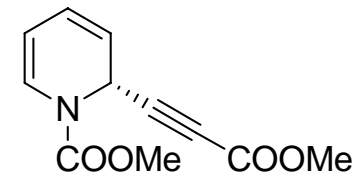

3b

(R)-Methyl 2-(2-(methoxycarbonyl)ethynyl)pyridine-1(2H)-carboxylate (3b): $74 \%$ yield. $[\alpha]_{\mathrm{D}}{ }^{29}+637\left(c 1.1, \mathrm{CHCl}_{3}\right) ;{ }^{1} \mathrm{H}$ NMR $\left(300 \mathrm{MHz}, \mathrm{CDCl}_{3}\right) \delta 6.75 \&$ 6.64 (due to rotamers, both d, $J=7.5 \mathrm{~Hz}, 1 \mathrm{H}$ ), 5.98 (m, 1H ), $5.71 \& 5.60$ (due to rotamers, both d, $J=6.3 \mathrm{~Hz}, 1 \mathrm{H}), 5.50(\mathrm{~m}, 1 \mathrm{H}), 5.28(\mathrm{~m}, 1 \mathrm{H}), 3.78(\mathrm{~s}, 3 \mathrm{H}), 3.69(\mathrm{~s}$, $3 \mathrm{H}) ;{ }^{13} \mathrm{C} \mathrm{NMR}\left(75 \mathrm{MHz}, \mathrm{CDCl}_{3}\right) \delta 153.4,153.1,125.2,123.9,116.0,105.3,84.2$, 74.3, 53.6, 52.6, 43.4; MS (ESI) $\mathrm{m} / \mathrm{z} 244.1(\mathrm{M}+\mathrm{Na})^{+}$; HRMS $\mathrm{m} / \mathrm{z}$ calcd for $\mathrm{C}_{11} \mathrm{H}_{11} \mathrm{NO}_{4} \mathrm{Na}(\mathrm{M}+\mathrm{Na})^{+}$244.0580, found 244.0589; HPLC DAICEL CHIRALPAK 
$\mathrm{AD}-\mathrm{H}$, hexane/2-proponal $=80 / 20$, flow rate: $0.8 \mathrm{~mL} / \mathrm{min}$, detector: $214 \mathrm{~nm}, \mathrm{t}_{\mathrm{R}}$ (minor)

$=8.1 \mathrm{~min}, \mathrm{t}_{\mathrm{R}}($ major $)=9.5 \mathrm{~min}, \mathrm{ee}=95 \%$.

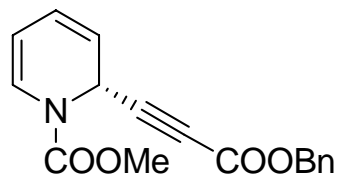

3c

(R)-Methyl 2-(2-((benzyloxy)carbonyl)ethynyl)pyridine-1(2H)-carboxylate (3c): $81 \%$ yield. $[\alpha]_{\mathrm{D}}{ }^{29}+549\left(\right.$ c $\left.0.27, \mathrm{CHCl}_{3}\right) ;{ }^{1} \mathrm{H} \mathrm{NMR}\left(300 \mathrm{MHz}, \mathrm{CDCl}_{3}\right) \delta 7.33(\mathrm{~m}$, 5H), $6.81 \& 6.67$ (due to rotamers, both d, $J=7.8 \mathrm{~Hz}, 1 \mathrm{H}), 6.03(\mathrm{~m}, 1 \mathrm{H}$ ), $5.77 \&$ 5.64 (due to rotamers, both $\mathrm{d}, J=6.0 \mathrm{~Hz}, 1 \mathrm{H}), 5.55(\mathrm{~m}, 1 \mathrm{H}), 5.38(\mathrm{~m}, 1 \mathrm{H}), 5.16(\mathrm{~s}$, 2H), $3.83(\mathrm{~s}, 3 \mathrm{H}) ;{ }^{13} \mathrm{C}$ NMR $\left(75 \mathrm{MHz}, \mathrm{CDCl}_{3}\right) \delta 153.1,153.0,134.5,128.7,128.6$, 125.0, 123.9, 116.0, 109.7, 105.5, 84.5, 74.6, 67.7, 53.7, 43.5; MS (ESI) m/z 320.1 (M $+\mathrm{Na})^{+}$; HRMS $m / z$ calcd for $\mathrm{C}_{17} \mathrm{H}_{15} \mathrm{NO}_{4} \mathrm{Na}(\mathrm{M}+\mathrm{Na})^{+}$320.0893, found 320.0891; HPLC DAICEL CHIRALPAK OD-H, hexane/2-proponal $=95 / 5$, flow rate: 0.8 $\mathrm{mL} / \mathrm{min}$, detector: $214 \mathrm{~nm}, \mathrm{t}_{\mathrm{R}}($ minor $)=22.0 \mathrm{~min}, \mathrm{t}_{\mathrm{R}}($ major $)=27.0 \mathrm{~min}$, ee $=86 \%$.

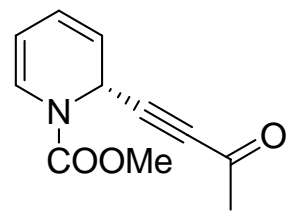

$3 d$

(R)-Methyl 2-(3-oxobut-1-ynyl)pyridine-1(2H)-carboxylate (3d): 69\% yield. $[\alpha]_{\mathrm{D}}{ }^{29}+594\left(c\right.$ 0.57, $\left.\mathrm{CHCl}_{3}\right) ;{ }^{1} \mathrm{H}$ NMR $\left(300 \mathrm{MHz}, \mathrm{CDCl}_{3}\right) \delta 6.81 \& 6.69$ (due to rotamers, both d, $J=7.5 \mathrm{~Hz}, 1 \mathrm{H}), 6.03(\mathrm{~m}, 1 \mathrm{H}), 5.79 \& 5.67$ (due to rotamers, both d, $J=6.3 \mathrm{~Hz}, 1 \mathrm{H}), 5.55(\mathrm{~m}, 1 \mathrm{H}), 5.36(\mathrm{~m}, 1 \mathrm{H}), 3.83(\mathrm{~s}, 3 \mathrm{H}), 2.3(\mathrm{~s}, 3 \mathrm{H}) ;{ }^{13} \mathrm{C} \mathrm{NMR}(75$ $\left.\mathrm{MHz}, \mathrm{CDCl}_{3}\right) \delta 184.1,153.3,125.3,123.9,116.3,105.3,87.9,82.2,53.6,43.6,32.5$; MS (ESI) $m / z 228.1(\mathrm{M}+\mathrm{Na})^{+} ;$HRMS $\mathrm{m} / \mathrm{z}$ calcd for $\mathrm{C}_{11} \mathrm{H}_{11} \mathrm{NO}_{3} \mathrm{Na}(\mathrm{M}+\mathrm{Na})^{+}$ 

$=98 / 2$, flow rate: $0.8 \mathrm{~mL} / \mathrm{min}$, detector: $214 \mathrm{~nm}, \mathrm{t}_{\mathrm{R}}($ minor $)=17.0 \mathrm{~min}, \mathrm{t}_{\mathrm{R}}($ major $)=$ $19.6 \min$, ee $=93 \%$.

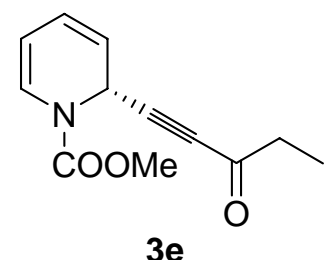

(R)-Methyl 2-(3-oxopent-1-ynyl)pyridine-1(2H)-carboxylate $\quad(3 e): \quad 65 \%$ yield. $[\alpha]_{\mathrm{D}}{ }^{29}+602\left(c 1.17, \mathrm{CHCl}_{3}\right) ;{ }^{1} \mathrm{H}$ NMR $\left(300 \mathrm{MHz}, \mathrm{CDCl}_{3}\right) \delta 6.81 \& 6.69$ (due to rotamers, both d, $J=7.5 \mathrm{~Hz}, 1 \mathrm{H}), 6.02(\mathrm{~m}, 1 \mathrm{H}), 5.79 \& 5.67$ (due to rotamers, both d, $J=6.3 \mathrm{~Hz}, 1 \mathrm{H}), 5.55(\mathrm{~m}, 1 \mathrm{H}), 5.36(\mathrm{~m}, 1 \mathrm{H}), 3.83(\mathrm{~s}, 3 \mathrm{H}), 2.56(\mathrm{q}, J=7.8 \mathrm{~Hz}, 2 \mathrm{H})$, $1.10(\mathrm{t}, J=7.5 \mathrm{~Hz}, 3 \mathrm{H}) ;{ }^{13} \mathrm{C} \mathrm{NMR}\left(75 \mathrm{MHz}, \mathrm{CDCl}_{3}\right) \delta 188.1,153.3,125.3,123.9$, $116.5,105.4,88.3,82.0,53.7,43.8,38.6,7.76 ; \mathrm{MS}(\mathrm{ESI}) \mathrm{m} / \mathrm{z} 242.1(\mathrm{M}+\mathrm{Na})^{+}$; HRMS $\mathrm{m} / \mathrm{z}$ calcd for $\mathrm{C}_{12} \mathrm{H}_{13} \mathrm{NO}_{3} \mathrm{Na}(\mathrm{M}+\mathrm{Na})^{+}$242.0788, found 242.0789; HPLC DAICEL CHIRALPAK AD-H, hexane/2-proponal = 100/1, flow rate: $0.8 \mathrm{~mL} / \mathrm{min}$, detector: $220 \mathrm{~nm}, \mathrm{t}_{\mathrm{R}}($ minor $)=27.9 \mathrm{~min}, \mathrm{t}_{\mathrm{R}}($ major $)=33.4 \mathrm{~min}$, ee $=99 \%$.

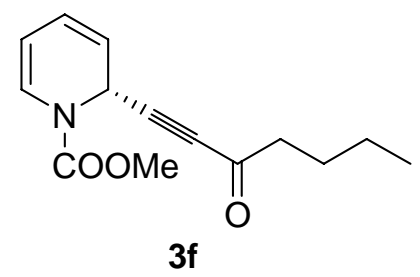

(R)-Methyl 2-(3-oxohept-1-ynyl)pyridine-1(2H)-carboxylate $\quad$ (3f): $\quad 70 \%$ yield. $[\alpha]_{\mathrm{D}}{ }^{29}+563\left(c 0.4, \mathrm{CHCl}_{3}\right) ;{ }^{1} \mathrm{H} \mathrm{NMR}\left(300 \mathrm{MHz}, \mathrm{CDCl}_{3}\right) \delta 6.81 \& 6.68$ (due to rotamers, both d, $J=7.5 \mathrm{~Hz}, 1 \mathrm{H}), 6.02(\mathrm{~m}, 1 \mathrm{H}), 5.79 \& 5.67$ (due to rotamers, both d, $J=6.0 \mathrm{~Hz}, 1 \mathrm{H}), 5.55(\mathrm{~m}, 1 \mathrm{H}), 5.34(\mathrm{~m}, 1 \mathrm{H}), 3.83(\mathrm{~s}, 3 \mathrm{H}), 2.53(\mathrm{t}, J=7.5 \mathrm{~Hz}, 2 \mathrm{H})$, $1.60(\mathrm{~m}, 2 \mathrm{H}), 1.34(\mathrm{~m}, 2 \mathrm{H}), 0.9(\mathrm{t}, J=7.2 \mathrm{~Hz}, 3 \mathrm{H}) ;{ }^{13} \mathrm{C} \mathrm{NMR}\left(75 \mathrm{MHz}, \mathrm{CDCl}_{3}\right) \delta$ $187.7,153.4,125.5,123.9,116.5,105.5,88.3,81.9,53.7,45.0,43.9,25.9,22.0,13.7$; 
MS (ESI) $m / z 270.1(\mathrm{M}+\mathrm{Na})^{+}$; HRMS m/z calcd for $\mathrm{C}_{14} \mathrm{H}_{17} \mathrm{NO}_{3} \mathrm{Na}(\mathrm{M}+\mathrm{Na})^{+}$ 270.1101, found 270.1101; HPLC DAICEL CHIRALPAK OD-H, hexane/2-proponal $=98 / 2$, flow rate: $0.5 \mathrm{~mL} / \mathrm{min}$, detector: $214 \mathrm{~nm}, \mathrm{t}_{\mathrm{R}}($ minor $)=13.6 \mathrm{~min}, \mathrm{t}_{\mathrm{R}}($ major $)=$ $14.6 \min$, ee $=91 \%$.

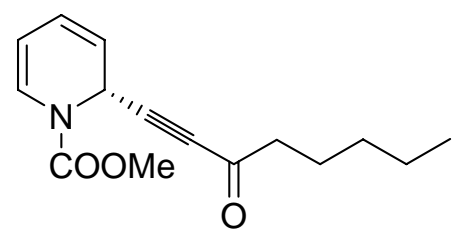

$3 g$

(R)-Methyl 2-(3-oxooct-1-ynyl)pyridine-1(2H)-carboxylate (3g): 68\% yield. $[\alpha]_{\mathrm{D}}{ }^{29}+558\left(\mathrm{c} 1.75, \mathrm{CHCl}_{3}\right) ;{ }^{1} \mathrm{H}$ NMR $\left(300 \mathrm{MHz}, \mathrm{CDCl}_{3}\right) \delta 6.78 \& 6.66$ (due to rotamers, both d, $J=7.2 \mathrm{~Hz}, 1 \mathrm{H}), 5.98(\mathrm{~m}, 1 \mathrm{H}), 5.76 \& 5.64$ (due to rotamers, both $\mathrm{d}$, $J=6.0 \mathrm{~Hz}, 1 \mathrm{H}), 5.56(\mathrm{~m}, 1 \mathrm{H}), 5.34(\mathrm{~m}, 1 \mathrm{H}), 3.83(\mathrm{~s}, 3 \mathrm{H}), 2.48(\mathrm{t}, J=7.8 \mathrm{~Hz}, 2 \mathrm{H})$, $1.60(\mathrm{~m}, 2 \mathrm{H}), 1.30(\mathrm{~m}, 4 \mathrm{H}), 0.84(\mathrm{t}, J=6.6 \mathrm{~Hz}, 3 \mathrm{H}) ;{ }^{13} \mathrm{C} \mathrm{NMR}\left(75 \mathrm{MHz}, \mathrm{CDCl}_{3}\right) \delta$ $188.0,153.0,125.0,123.0,116.0,105.0,88.1,81.1,53.6,45.2,43.5,30.9,23.3,22.2$, 13.7; MS (ESI) $m / z 284.1(\mathrm{M}+\mathrm{Na})^{+} ;$HRMS $m / z$ calcd for $\mathrm{C}_{15} \mathrm{H}_{19} \mathrm{NO}_{3} \mathrm{Na}(\mathrm{M}+\mathrm{Na})^{+}$ 284.1257, found 284.1252; HPLC DAICEL CHIRALPAK OD-H, hexane/2-proponal $=98 / 2$, flow rate: $0.3 \mathrm{~mL} / \mathrm{min}$, detector: $214 \mathrm{~nm}, \mathrm{t}_{\mathrm{R}}($ minor $)=28.3 \mathrm{~min}, \mathrm{t}_{\mathrm{R}}($ major $)=$ $30.3 \min$, ee $=90 \%$.

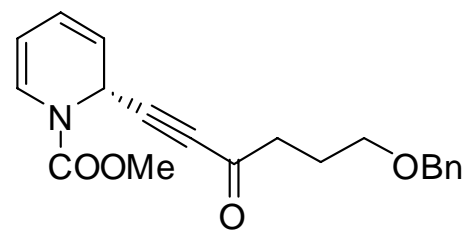

$3 h$

(R)-Methyl 2-(6-(benzyloxy)-3-oxohex-1-ynyl)pyridine-1(2H)-carboxylate (3h): $70 \%$ yield. $[\alpha]_{\mathrm{D}}^{29}+427\left(c 0.8, \mathrm{CHCl}_{3}\right) ;{ }^{1} \mathrm{H}$ NMR $\left(300 \mathrm{MHz}, \mathrm{CDCl}_{3}\right) \delta 7.31(\mathrm{~m}$, $5 \mathrm{H}$ ), $6.81 \& 6.68$ (due to rotamers, both $\mathrm{d}, J=7.5 \mathrm{~Hz}, 1 \mathrm{H}), 6.03(\mathrm{~m}, 1 \mathrm{H}), 5.79 \& 5.66$ 
(due to rotamers, both d, $J=5.4 \mathrm{~Hz}, 1 \mathrm{H}), 5.55(\mathrm{~m}, 1 \mathrm{H}), 5.34(\mathrm{~m}, 1 \mathrm{H}), 4.47(\mathrm{~s}, 2 \mathrm{H})$, $3.83(\mathrm{~s}, 3 \mathrm{H}), 3.47(\mathrm{t}, J=6.0 \mathrm{~Hz}, 2 \mathrm{H}), 2.67(\mathrm{t}, J=7.5 \mathrm{~Hz}, 2 \mathrm{H}), 1.93(\mathrm{~m}, 2 \mathrm{H}) ;$ MS (ESI) $m / z$ 362.2 $(\mathrm{M}+\mathrm{Na})^{+} ;$HRMS m/z calcd for $\mathrm{C}_{20} \mathrm{H}_{21} \mathrm{NO}_{4} \mathrm{Na} 362.1363(\mathrm{M}+\mathrm{Na})^{+}$, found 362.1375; HPLC DAICEL CHIRALPAK AD-H, hexane/2-proponal = 90/10, flow rate: $0.8 \mathrm{~mL} / \mathrm{min}$, detector: $214 \mathrm{~nm}, \mathrm{t}_{\mathrm{R}}($ minor $)=16.0 \mathrm{~min}, \mathrm{t}_{\mathrm{R}}($ major $)=17.6 \mathrm{~min}$, ee $=$ $77 \%$.

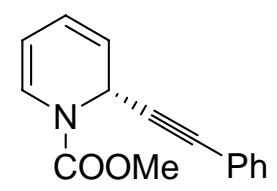

3i

(R)-Methyl 2-(2-phenylethynyl)pyridine-1(2H)-carboxylate (3i): 75\% yield. ${ }^{1} \mathrm{H}$ NMR $\left(300 \mathrm{MHz}, \mathrm{CDCl}_{3}\right) \delta 7.39(\mathrm{~m}, 2 \mathrm{H}), 7.25(\mathrm{~m}, 3 \mathrm{H}), 6.84 \& 6.70$ (due to rotamers, both d, $J=7.2 \mathrm{~Hz}, 1 \mathrm{H}), 5.99(\mathrm{~m}, 1 \mathrm{H}), 5.83 \& 5.68$ (due to rotamers, both d, $J=6.0 \mathrm{~Hz}, 1 \mathrm{H}), 5.62(\mathrm{~m}, 1 \mathrm{H}), 5.35(\mathrm{~m}, 1 \mathrm{H}), 3.38(\mathrm{~s}, 3 \mathrm{H}) ;{ }^{13} \mathrm{C} \mathrm{NMR}\left(75 \mathrm{MHz}, \mathrm{CDCl}_{3}\right)$ $\delta 153.4,131.7,131.4,128.1,127.8,125.1,122.3,118.6,105.3,86.3,82.8,53.3,44.1$; MS (ESI) m/z $262.1(\mathrm{M}+\mathrm{Na})^{+} ;$HRMS m/z calcd for $\mathrm{C}_{15} \mathrm{H}_{13} \mathrm{NO}_{2} \mathrm{Na}(\mathrm{M}+\mathrm{Na})^{+}$ 262.0838, found 262.0841; HPLC DAICEL CHIRALPAK AD-H, hexane/2-proponal $=80 / 20$, flow rate: $0.8 \mathrm{~mL} / \mathrm{min}$, detector: $214 \mathrm{~nm}, \mathrm{t}_{\mathrm{R}}($ minor $)=6.93 \mathrm{~min}, \mathrm{t}_{\mathrm{R}}($ major $)=$ $7.34 \min$, ee $=1 \%$.

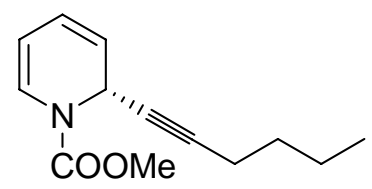

3j

(R)-Methyl 2-(hex-1-ynyl)pyridine-1(2H)-carboxylate (3j): 63\% yield. ${ }^{1} \mathrm{H}$ NMR (300 MHz, $\left.\mathrm{CDCl}_{3}\right) \delta 6.70(\mathrm{~m}, 1 \mathrm{H}), 5.86(\mathrm{~m}, 1 \mathrm{H}), 5.53(\mathrm{~m}, 1 \mathrm{H}), 5.43(\mathrm{~m}, 1 \mathrm{H})$, $5.27(\mathrm{~m}, 1 \mathrm{H}), 3.73(\mathrm{~s}, 3 \mathrm{H}), 2.11(\mathrm{t}, J=7.2 \mathrm{~Hz}, 2 \mathrm{H}), 1.36(\mathrm{~m}, 2 \mathrm{H}), 1.27(\mathrm{~m}, 2 \mathrm{H}), 0.81(\mathrm{t}$, 
$J=7.2 \mathrm{~Hz}, 3 \mathrm{H}) ;{ }^{13} \mathrm{C} \mathrm{NMR}\left(75 \mathrm{MHz}, \mathrm{CDCl}_{3}\right) \delta 153.0,124.8,121.4,119.8,105.4,83.9$, 73.3, 53.4, 43.6, 30.0, 21.8, 18.5, 13.6; MS (ESI) $m / z$ 242.1 $(\mathrm{M}+\mathrm{Na})^{+}$; HRMS m/z calcd for $\mathrm{C}_{13} \mathrm{H}_{17} \mathrm{NO}_{2} \mathrm{Na}(\mathrm{M}+\mathrm{Na})^{+}$242.1151, found 242.1164; HPLC DAICEL CHIRALPAK AD-H, hexane $/ 2$-proponal $=90 / 10$, flow rate: $0.8 \mathrm{~mL} / \mathrm{min}$, detector: $214 \mathrm{~nm}, \mathrm{t}_{\mathrm{R}}($ minor $)=5.96 \mathrm{~min}, \mathrm{t}_{\mathrm{R}}($ major $)=6.26 \mathrm{~min}, \mathrm{ee}=11 \%$.

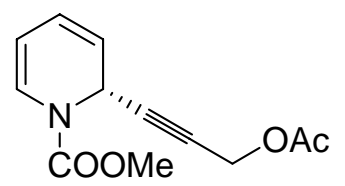

3k

(R)-Methyl 2-(3-acetoxyprop-1-ynyl)pyridine-1(2H)-carboxylate (3k): 77\% yield. ${ }^{1} \mathrm{H}$ NMR (300 MHz, $\left.\mathrm{CDCl}_{3}\right) \delta 6.75(\mathrm{~m}, 1 \mathrm{H}), 5.90(\mathrm{~m}, 1 \mathrm{H}), 5.63(\mathrm{~m}, 1 \mathrm{H}), 5.55$ $(\mathrm{m}, 1 \mathrm{H}), 5.33(\mathrm{~m}, 1 \mathrm{H}), 4.68(\mathrm{~s}, 2 \mathrm{H}), 3.83(\mathrm{~s}, 3 \mathrm{H}), 2.10(\mathrm{~s}, 3 \mathrm{H}) ;{ }^{13} \mathrm{C} \mathrm{NMR}(75 \mathrm{MHz}$, $\left.\mathrm{CDCl}_{3}\right) \delta 170.2,153.0,124.6,122.4,118.3,105.4,84.0,74.1,53.6,52.2,43.5,20.7$ MS (ESI) $m / z 258.1(\mathrm{M}+\mathrm{Na})^{+} ;$HRMS $\mathrm{m} / \mathrm{z}$ calcd for $\mathrm{C}_{12} \mathrm{H}_{13} \mathrm{NO}_{4} \mathrm{Na}(\mathrm{M}+\mathrm{Na})^{+}$ 258.0737, found 258.0749; HPLC DAICEL CHIRALPAK AD-H, hexane/2-proponal $=90 / 10$, flow rate: $0.8 \mathrm{~mL} / \mathrm{min}$, detector: $214 \mathrm{~nm}, \mathrm{t}_{\mathrm{R}}($ minor $)=11.9 \min , \mathrm{t}_{\mathrm{R}}($ major $)=$ $13.2 \mathrm{~min}, \mathrm{ee}=3 \%$.

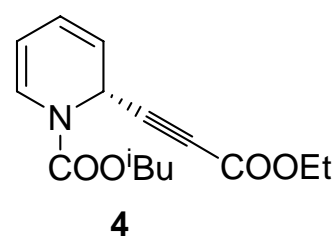

(R)-Butyl 2-(2-(ethoxycarbonyl)ethynyl)pyridine-1(2H)-carboxylate (4): $72 \%$ yield. ${ }^{1} \mathrm{H}$ NMR $\left(300 \mathrm{MHz}, \mathrm{CDCl}_{3}\right) \delta 6.82 \& 6.73$ (due to rotamers, both $\mathrm{d}, J=$ $6.0 \mathrm{~Hz}, 1 \mathrm{H}), 6.06(\mathrm{~m}, 1 \mathrm{H}), 5.79 \& 5.68$ (due to rotamers, both d, $J=5.4 \mathrm{~Hz}, 1 \mathrm{H}$ ), $5.57(\mathrm{~m}, 1 \mathrm{H}), 5.35(\mathrm{~m}, 1 \mathrm{H}), 4.22(\mathrm{t}, J=7.2 \mathrm{~Hz}, 2 \mathrm{H}), 4.04(\mathrm{~m}, 2 \mathrm{H}), 2.04(\mathrm{~m}, 1 \mathrm{H}), 1.28$ $(\mathrm{t}, J=7.2 \mathrm{~Hz}, 3 \mathrm{H}), 0.96(\mathrm{~d}, J=6.9 \mathrm{~Hz}, 6 \mathrm{H}) ;{ }^{13} \mathrm{C} \mathrm{NMR}\left(75 \mathrm{MHz}, \mathrm{CDCl}_{3}\right) \delta 153.2$, 
$152.9,125.5,124.0,116.2,105.2,83.9,75.7,72.9,62.0,43.8,43.2,28.0,19.1,13.9$; MS (ESI) $\mathrm{m} / \mathrm{z} 300.1(\mathrm{M}+\mathrm{Na})^{+}$; HRMS $\mathrm{m} / \mathrm{z}$ calcd for $\mathrm{C}_{15} \mathrm{H}_{19} \mathrm{NO}_{4} \mathrm{Na}(\mathrm{M}+\mathrm{Na})^{+}$ 300.1206, found 300.1217; HPLC DAICEL CHIRALPAK OD-H, hexane/2-proponal $=98 / 2$, flow rate: $0.7 \mathrm{~mL} / \mathrm{min}$, detector: $214 \mathrm{~nm}, \mathrm{t}_{\mathrm{R}}($ minor $)=12.6 \mathrm{~min}, \mathrm{t}_{\mathrm{R}}($ major $)=$ $14.6 \mathrm{~min}, \mathrm{ee}=86 \%$.

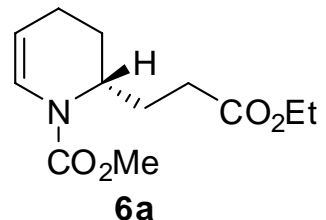

(R)-Methyl 2-(3-ethoxy-3-oxopropyl)-3,4-dihydropyridine-1(2H)carboxylate (6a): To a solution of 3a $(77 \mathrm{mg}, 0.33 \mathrm{mmol})$ in $\mathrm{MeOH}(5 \mathrm{~mL})$ was added $10 \% \mathrm{Pd} / \mathrm{C}(7 \mathrm{mg})$. The reaction mixture was stirred under $\mathrm{H}_{2}(1 \mathrm{~atm})$ for $2 \mathrm{~h}$. The catalyst was filtered off and flash chromatography (hexanes/EtOAc 20/1 to 15/1) gave 6a (55 mg, 70\%) as a colorless oil. $[\alpha]_{\mathrm{D}}^{23}+93.8\left(\right.$ c $\left.0.95, \mathrm{CHCl}_{3}\right)$; IR (film) 2956, $2850,1735,1707,1654,1445,1413,1362,1237,1190,1117,1076,945,769,720$ $\mathrm{cm}^{-1} ;{ }^{1} \mathrm{H} \mathrm{NMR}\left(300 \mathrm{MHz}, \mathrm{CDCl}_{3}\right) \delta 6.78 \& 6.64$ (due to rotamers, both $\mathrm{d}, J=8.4 \mathrm{~Hz}$, $1 \mathrm{H}$ ), 4.98-4.90 \& 4.90-4.81 (due to rotamers, both $\mathrm{m}, 1 \mathrm{H}$ ), 4.40-4.31 \& 4.31-4.21 (due to rotamers, both $\mathrm{m}, 1 \mathrm{H}), 4.12(\mathrm{q}, J=6.9 \mathrm{~Hz}, 2 \mathrm{H}), 3.73(\mathrm{~s}, 3 \mathrm{H}), 2.41-2.25(\mathrm{~m}$, 2H), 2.10-1.96 (m, 2H), 1.96-1.82 (m, 1H), 1.81-1.68 (m, 3H), $1.24(\mathrm{t}, J=6.9 \mathrm{~Hz}$, $3 \mathrm{H}) ;{ }^{13} \mathrm{C} \mathrm{NMR}\left(75 \mathrm{MHz}, \mathrm{CDCl}_{3}\right) \delta 173.3,153.9,123.8 \& 123.4$ (due to rotamers), $106.3 \& 106.0$ (due to rotamers), $60.3,52.8 \& 52.7$ (due to rotamers), 49.7, $31.2 \&$ 30.8 (due to rotamers), 26.1, 24.8, $17.5 \& 17.4$ (due to rotamers), 14.2; MS (ESI) $\mathrm{m} / \mathrm{z}$ $242.1(\mathrm{M}+\mathrm{H})^{+}, 264.0(\mathrm{M}+\mathrm{Na})^{+} ;$HRMS m/z calcd for $\mathrm{C}_{12} \mathrm{H}_{19} \mathrm{NO}_{4} \mathrm{Na}(\mathrm{M}+\mathrm{Na})^{+}$ 264.1206, found 264.1210. 


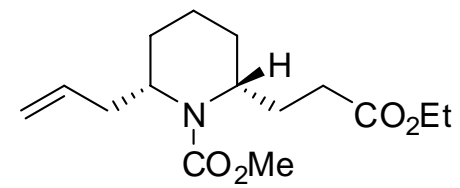

$7 a$

\section{(2S,6R)-Methyl 2-allyl-6-(3-ethoxy-3-oxopropyl)piperidine-1-carboxylate}

(7a): To a solution of $6 \mathbf{6}(51 \mathrm{mg}, 0.21 \mathrm{mmol})$ in $\mathrm{CH}_{2} \mathrm{Cl}_{2}(2 \mathrm{~mL})$ at $-20{ }^{\circ} \mathrm{C}$ was added allyltrimethylsilane $(0.20 \mathrm{~mL}, 1.26 \mathrm{mmol})$ followed by TFA $(0.065 \mathrm{~mL}, 0.86 \mathrm{mmol})$. After $2 \mathrm{~h}$ at $-20^{\circ} \mathrm{C}$, another portion of allyltrimethylsilane $(0.10 \mathrm{~mL}, 0.63 \mathrm{mmol})$ and TFA $(0.033 \mathrm{~mL}, 0.43 \mathrm{mmol})$ was added. The mixture was stirred at $-20{ }^{\circ} \mathrm{C}$ for another $2 \mathrm{~h}$ and then warmed to $0{ }^{\circ} \mathrm{C}$. The reaction was quenched with saturated $\mathrm{NaHCO}_{3}$ and extracted with $\mathrm{CH}_{2} \mathrm{Cl}_{2}$. The combined organic extracts were washed with saturated brine, dried over $\mathrm{Na}_{2} \mathrm{SO}_{4}$ and concentrated. Flash chromatography (hexanes/EtOAc $20 / 1$ to $15 / 1)$ gave 7 a $(52 \mathrm{mg}, 87 \%)$ as a colorless oil. $[\alpha]_{\mathrm{D}}{ }^{23}+7.0\left(\right.$ c $\left.1.0, \mathrm{CH}_{2} \mathrm{Cl}_{2}\right)$; IR (film) 2943, 2870, 1736, 1662, 1642, 1444, 1409, 1365, 1324, 1272, 1187, 1103, 1026, 916, $773 \mathrm{~cm}^{-1} ;{ }^{1} \mathrm{H}$ NMR $\left(300 \mathrm{MHz}, \mathrm{CDCl}_{3}\right) \delta$ 5.84-5.68 (m, 1H), 5.11-5.00 (m, 2H), 4.31-4.08 (m, 4H), $3.67(\mathrm{~s}, 3 \mathrm{H}), 2.41-2.24(\mathrm{~m}, 4 \mathrm{H}), 2.00-1.78(\mathrm{~m}, 2 \mathrm{H}), 1.76-1.40(\mathrm{~m}$, $6 \mathrm{H}), 1.26(\mathrm{t}, J=7.2 \mathrm{~Hz}, 3 \mathrm{H}) ;{ }^{13} \mathrm{C} \mathrm{NMR}\left(100 \mathrm{MHz}, \mathrm{CDCl}_{3}\right) \delta 173.3,156.7,136.0$, 117.0, 60.3, 52.4, 50.1, 49.8, 39.1, 32.0, 29.8, 28.1, 26.7, 14.2, 14.0; MS (ESI) m/z $284.2(\mathrm{M}+\mathrm{H})^{+}, 306.1(\mathrm{M}+\mathrm{Na})^{+} ;$HRMS $m / z$ calcd for $\mathrm{C}_{15} \mathrm{H}_{25} \mathrm{NO}_{4} \mathrm{Na}(\mathrm{M}+\mathrm{Na})^{+}$ 306.1676 , found 306.1683 .

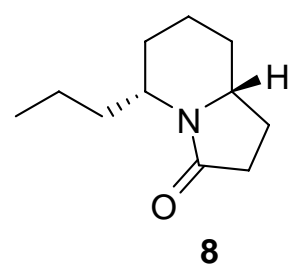

(5R,8aR)-5-Propylhexahydroindolizin-3(5H)-one (8): To a solution of 7a (62 $\mathrm{mg}, 0.21 \mathrm{mmol})$ in $\mathrm{MeOH}(3 \mathrm{~mL})$ was added $10 \% \mathrm{Pd} / \mathrm{C}(7 \mathrm{mg})$. The reaction 
mixture was stirred under $\mathrm{H}_{2}(1 \mathrm{~atm})$ for $1 \mathrm{~h}$. The catalyst was filtered off and concentration gave a colorless oil, which was dissolved in $5 \mathrm{~mL}$ of $\mathrm{MeCN}$. To this solution was added $\mathrm{NaI}(157 \mathrm{mg}, 1.05 \mathrm{mmol})$ and TMSCl $(0.14 \mathrm{~mL}, 1.07 \mathrm{mmol})$. The mixture was refluxed for $1 \mathrm{~h}$ under darkness and then cooled to room temperature. To the reaction mixture was added $\mathrm{MeOH}(1 \mathrm{~mL})$, then stirred for $30 \mathrm{~min}$. The mixture was partitioned between brine and $\mathrm{CH}_{2} \mathrm{Cl}_{2}$. The aqueous phase was extracted with $\mathrm{CH}_{2} \mathrm{Cl}_{2}$. The combined organic extracts were washed with saturated $\mathrm{Na}_{2} \mathrm{~S}_{2} \mathrm{O}_{3}$, dried over $\mathrm{Na}_{2} \mathrm{SO}_{4}$ and concentrated.

To a solution of the above residue in toluene $(6 \mathrm{~mL})$ was added $\mathrm{AlMe}_{3}(2.0 \mathrm{M}$ in toluene, $0.15 \mathrm{~mL}, 0.30 \mathrm{mmol})$. The mixture was stirred at $80^{\circ} \mathrm{C}$ overnight, cooled to room temperature and poured into $1 \mathrm{~N} \mathrm{HCl}(25 \mathrm{~mL})$. The mixture was extracted with $\mathrm{CH}_{2} \mathrm{Cl}_{2}$, washed with brine and concentrated. Flash chromatography (EtOAc) gave 8 (25 mg, 65\% for 3 steps $)$ as a colorless oil. $[\alpha]_{\mathrm{D}}{ }^{23}-27.2\left(\right.$ c $\left.1.1, \mathrm{CH}_{2} \mathrm{Cl}_{2}\right) ;{ }^{1} \mathrm{H}$ NMR (300 MHz, $\left.\mathrm{CDCl}_{3}\right)$ $\delta 3.44-3.33(\mathrm{~m}, 1 \mathrm{H}), 3.23-3.13(\mathrm{~m}, 1 \mathrm{H}), 2.40-2.27(\mathrm{~m}, 3 \mathrm{H})$, 2.16-2.03 (m, 1H), 1.90- $1.60(\mathrm{~m}, 4 \mathrm{H}), 1.59-1.22(\mathrm{~m}, 6 \mathrm{H}), 0.93(\mathrm{t}, J=7.2 \mathrm{~Hz}, 3 \mathrm{H})$; ${ }^{13} \mathrm{C}$ NMR $\left(100 \mathrm{MHz}, \mathrm{CDCl}_{3}\right) \delta 174.3,59.7,57.3,34.6,32.0,31.8,29.5,25.1,22.7$, 20.1, 14.1; MS (ESI) m/z 182.1 (M+H) ${ }^{+}, 204.1(\mathrm{M}+\mathrm{Na})^{+}$.

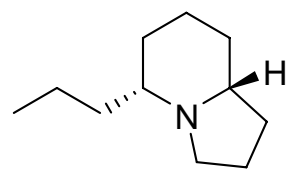

$(-)-167 B(9)$

(-)-167B (9): To a solution of $8(25 \mathrm{mg}, 0.14 \mathrm{mmol})$ in dry ether $(3 \mathrm{~mL})$ at 0 ${ }^{\circ} \mathrm{C}$ was added $\mathrm{LiAlH}_{4}(21 \mathrm{mg}, 0.54 \mathrm{mmol})$. The suspension was refluxed for $3 \mathrm{~h}$ and cooled to $0{ }^{\circ} \mathrm{C}$. Water $(0.025 \mathrm{~mL}), 15 \% \mathrm{NaOH}(0.025 \mathrm{~mL})$ and water $(0.075 \mathrm{~mL})$ were sequentially added. The resulting suspension was stirred for $30 \mathrm{~min}$, filtered through 
celite and dried over $\mathrm{Na}_{2} \mathrm{SO}_{4}$. Concentration gave (-)-167B (9) (19 mg, 82\%) as a yellow oil. $[\alpha]_{\mathrm{D}}{ }^{23}-102.8\left(c 0.88, \mathrm{CH}_{2} \mathrm{Cl}_{2}\right) ;{ }^{1} \mathrm{H}$ NMR $\left(500 \mathrm{MHz}, \mathrm{CDCl}_{3}\right) \delta 3.25(\mathrm{dt}, J=$ 8.8, 2.2 Hz, 1H), $1.95(\mathrm{t}, J=8.9 \mathrm{~Hz}, 1 \mathrm{H}), 1.88-1.52(\mathrm{~m}, 8 \mathrm{H}), 1.50-1.06(\mathrm{~m}, 8 \mathrm{H}), 0.90$ $(\mathrm{t}, J=7.1 \mathrm{~Hz}, 3 \mathrm{H}) ;{ }^{13} \mathrm{C}$ NMR $\left(125 \mathrm{MHz}, \mathrm{CDCl}_{3}\right) \delta 65.0,63.7,51.6,36.9,31.0,30.9$, 24.7, 20.4, 19.1, 14.5; MS (ESI) $m / z$ 168.1 $(\mathrm{M}+\mathrm{H})^{+}$.

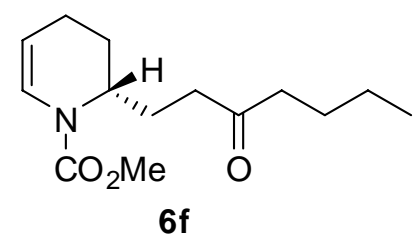

(R)-Methyl 2-(3-oxoheptyl)-3,4-dihydropyridine-1(2H)-carboxylate (6f):

Following the same procedure from 3a to $\mathbf{6 a}, \mathbf{6 f}$ was obtained from $3 \mathbf{f}$ in $72 \%$ yield. $[\alpha]_{\mathrm{D}}^{23}+93.4\left(c\right.$ 1.2, $\left.\mathrm{CHCl}_{3}\right)$; IR (film) 2960, 2874, 1712, 1655, 1444, 1413, 1361, 1237, 1191, 1117, 1061, 769, $719 \mathrm{~cm}^{-1} ;{ }^{1} \mathrm{H} \mathrm{NMR}\left(300 \mathrm{MHz}, \mathrm{CDCl}_{3}\right) \delta 6.75 \& 6.61$ (due to rotamers, both d, $J=9.3 \& 8.1 \mathrm{~Hz}, 1 \mathrm{H}$ ), 4.96-4.94 \& 4.88-4.82 (due to rotamers, both $\mathrm{m}, 1 \mathrm{H}), 4.28-4.24 \& 4.23-4.17$ (due to rotamers, both $\mathrm{m}, 1 \mathrm{H}), 3.72(\mathrm{~s}$, $3 \mathrm{H}), 2.53-2.36(\mathrm{~m}, 4 \mathrm{H}), 2.08-1.90(\mathrm{~m}, 2 \mathrm{H}), 1.83-1.63(\mathrm{~m}, 4 \mathrm{H}), 1.57-1.47(\mathrm{~m}, 2 \mathrm{H})$, 1.31-1.23 (m, 2H), $0.87(\mathrm{t}, J=7.2 \mathrm{~Hz}, 3 \mathrm{H}) ;{ }^{13} \mathrm{C} \mathrm{NMR}\left(100 \mathrm{MHz}, \mathrm{CDCl}_{3}\right) \delta$ 210.7, 154.0, $123.7 \& 123.3$ (due to rotamers), $106.5 \& 106.2$ (due to rotamers), 52.8, 49.8, $42.5,39.4 \& 38.9$ (due to rotamers), 25.9, 25.1, 24.9, 22.3, 17.6, 13.8; MS (ESI) $\mathrm{m} / \mathrm{z}$ $254.1(\mathrm{M}+\mathrm{H})^{+}, 276.1(\mathrm{M}+\mathrm{Na})^{+} ;$HRMS m/z calcd for $\mathrm{C}_{14} \mathrm{H}_{24} \mathrm{NO}_{3}(\mathrm{M}+\mathrm{H})^{+}$ 254.1751, found 254.1762 .<smiles>C=CC[C@@H]1CCC[C@H](CCC(=O)CCCC)N1C(C)=O</smiles>

$7 f$ 
Following the same procedure from 6a to 7a, 7f was obtained from $\mathbf{6 f}$ in $94 \%$ yield. $[\alpha]_{\mathrm{D}}{ }^{26}+12.0\left(c 1.1, \mathrm{CHCl}_{3}\right)$; IR (film) 2958, 2874, 1695, 1444, 1409, 1365, 1324, $1272,1191,1100,915,772 \mathrm{~cm}^{-1} ;{ }^{1} \mathrm{H}$ NMR $\left(300 \mathrm{MHz}, \mathrm{CDCl}_{3}\right) \delta 5.78-5.67(\mathrm{~m}, 1 \mathrm{H})$, 5.06-5.00 (m, 2H), 4.26- $4.22(\mathrm{~m}, 1 \mathrm{H}) 4.17-4.13(\mathrm{~m}, 1 \mathrm{H}), 3.67(\mathrm{~s}, 3 \mathrm{H}), 2.49-2.29(\mathrm{~m}$, $6 \mathrm{H}), 1.85-1.78(\mathrm{~m}, 2 \mathrm{H}), 1.76-1.41(\mathrm{~m}, 8 \mathrm{H}), 1.36-1.23(\mathrm{~m}, 2 \mathrm{H}), 0.89(\mathrm{t}, J=7.2 \mathrm{~Hz}$, $3 \mathrm{H}) ;{ }^{13} \mathrm{C} \mathrm{NMR}\left(100 \mathrm{MHz}, \mathrm{CDCl}_{3}\right) \delta 210.6,156.8,136.1,116.9,52.4,50.2,49.9,42.5$, 40.3, 39.2, 28.5, 28.3, 26.8, 25.9, 22.3, 14.1, 13.8; MS (ESI) $\mathrm{m} / \mathrm{z} 296.2(\mathrm{M}+\mathrm{H})^{+}$, $318.2(\mathrm{M}+\mathrm{Na})^{+}$; HRMS $\mathrm{m} / \mathrm{z}$ calcd for $\mathrm{C}_{17} \mathrm{H}_{30} \mathrm{NO}_{3}(\mathrm{M}+\mathrm{H})^{+}$296.2220, found 296.2231.

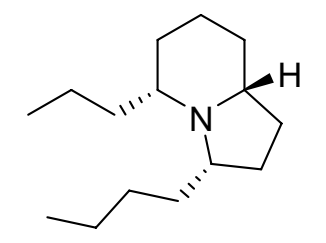

$(-)-5 Z, 9 Z-223 A B(10)$

(-)-(5Z, 9Z)-223AB (10): To a solution of $7 \mathbf{f}(20 \mathrm{mg}, 0.068 \mathrm{mmol})$ in $\mathrm{MeOH}$ $(1 \mathrm{~mL})$ was added $10 \% \mathrm{Pd} / \mathrm{C}(2 \mathrm{mg})$. The reaction mixture was stirred under $\mathrm{H}_{2}(1$ atm) for $1 \mathrm{~h}$. The catalyst was filtered off and the filtrate was concentrated to give a colorless oil, which was dissolved in $2 \mathrm{~mL}$ of $\mathrm{MeCN}$. To this solution was added $\mathrm{NaI}$ (51 $\mathrm{mg}, 0.34 \mathrm{mmol})$ and $\mathrm{TMSCl}(0.48 \mathrm{~mL}, 0.37 \mathrm{mmol})$. The resultant mixture was refluxed for $1 \mathrm{~h}$ under darkness and then cooled to room temperature. To the reaction mixture was added $\mathrm{MeOH}(0.5 \mathrm{~mL})$, then stirred for $30 \mathrm{~min}$. The mixture was partitioned between brine and $\mathrm{CH}_{2} \mathrm{Cl}_{2}$. The aqueous phase was extracted with $\mathrm{CH}_{2} \mathrm{Cl}_{2}$. The combined organic extracts were washed with saturated $\mathrm{Na}_{2} \mathrm{~S}_{2} \mathrm{O}_{3}$, dried over $\mathrm{Na}_{2} \mathrm{SO}_{4}$ and concentrated.

A solution of the above residue in $\operatorname{THF}(1.5 \mathrm{~mL})$ and $1 \mathrm{~N} \mathrm{HCl}(0.5 \mathrm{~mL})$ was 
stirred for $1 \mathrm{~d}$. The mixture was then washed with ether, basified by saturated $\mathrm{K}_{2} \mathrm{CO}_{3}$, and extracted with $\mathrm{CH}_{2} \mathrm{Cl}_{2}(10 \mathrm{~mL} \times 2)$. The combined organic phase was dried over $\mathrm{Na}_{2} \mathrm{SO}_{4}$ and concentrated.

To a solution of the above residue in $\mathrm{MeOH}(1 \mathrm{~mL})$ was added $\mathrm{PtO}_{2}$. The mixture was then stirred under $\mathrm{H}_{2}(1 \mathrm{~atm})$ for $2 \mathrm{~d}$. The catalyst was filtered off and the mixture was then acidified by $1 \mathrm{~N} \mathrm{HCl}$, washed with ether, basified by saturated $\mathrm{K}_{2} \mathrm{CO}_{3}$ and extracted with $\mathrm{CH}_{2} \mathrm{Cl}_{2}(10 \mathrm{~mL} \times 2)$. The combined organic phase was dried over $\mathrm{Na}_{2} \mathrm{SO}_{4}$ and concentrated. Flash chromatography on neutral $\mathrm{Al}_{2} \mathrm{O}_{3}$ eluting with EtOAc gave (-)-(5Z, 9Z)-223AB (10) (9 mg, 60\% for 4 steps) as a yellow oil. $[\alpha]_{\mathrm{D}}{ }^{23}-11.1(c \quad 0.20, \mathrm{MeOH}) ;{ }^{1} \mathrm{H}$ NMR $\left(500 \mathrm{MHz}, \mathrm{CDCl}_{3}\right) \delta 2.35-2.30(\mathrm{~m}, 1 \mathrm{H})$, 2.15-2.06 (m, 2H), 1.80-1.54 (m, 4H), 1.50-1.10 (m, 16H), 0.98-0.86 (m, 6H); ${ }^{13} \mathrm{C}$ NMR (125 MHz, $\left.\mathrm{CDCl}_{3}\right) \delta 67.6,65.1,62.2,39.9,38.1$ 32.0, 31.0, 30.5, 29.9, 29.3, 25.0, 22.9, 19.3, 14.5, 14.2; MS (ESI) $\mathrm{m} / \mathrm{z} 224.2(\mathrm{M}+\mathrm{H})^{+}$. 


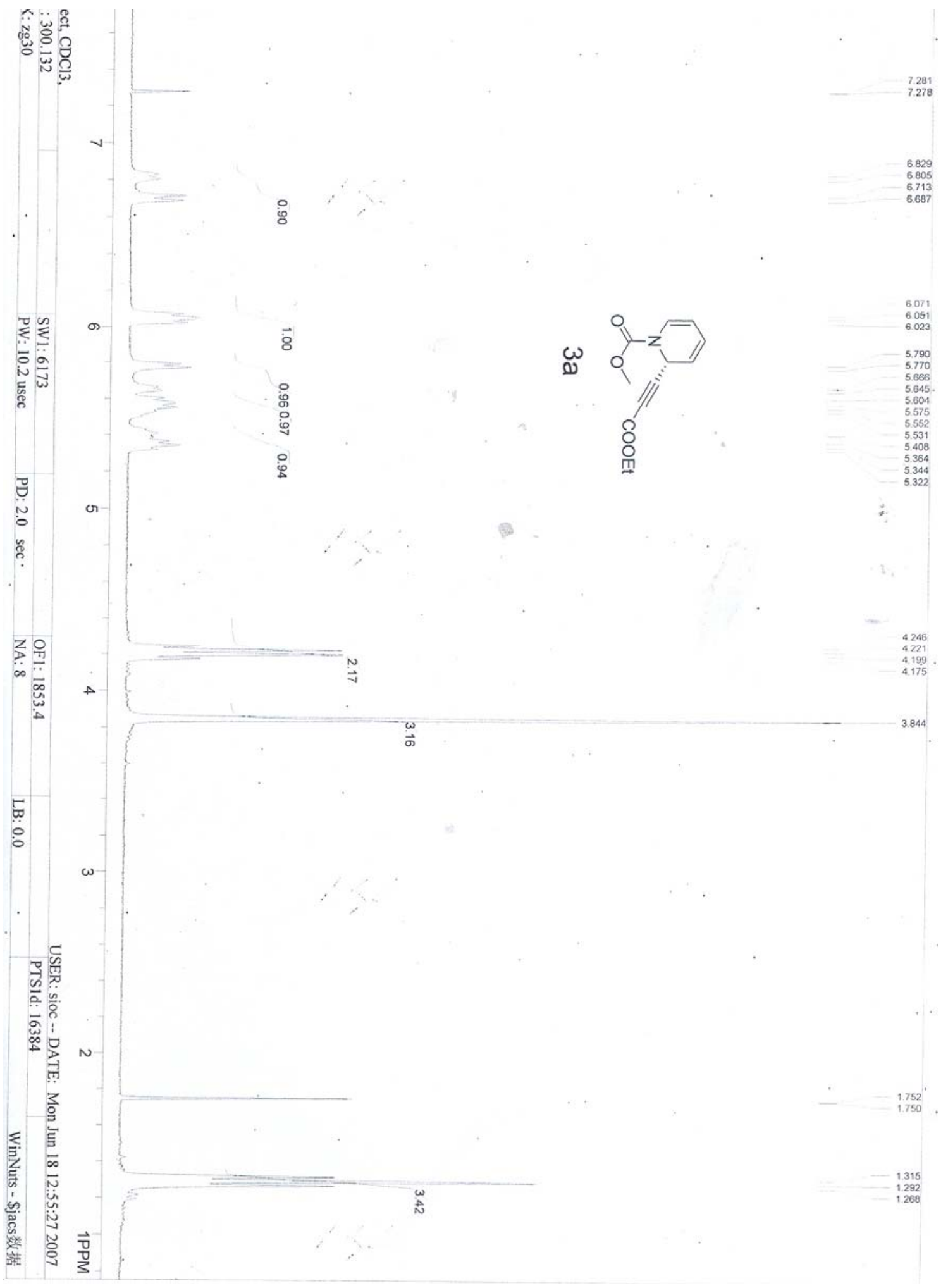



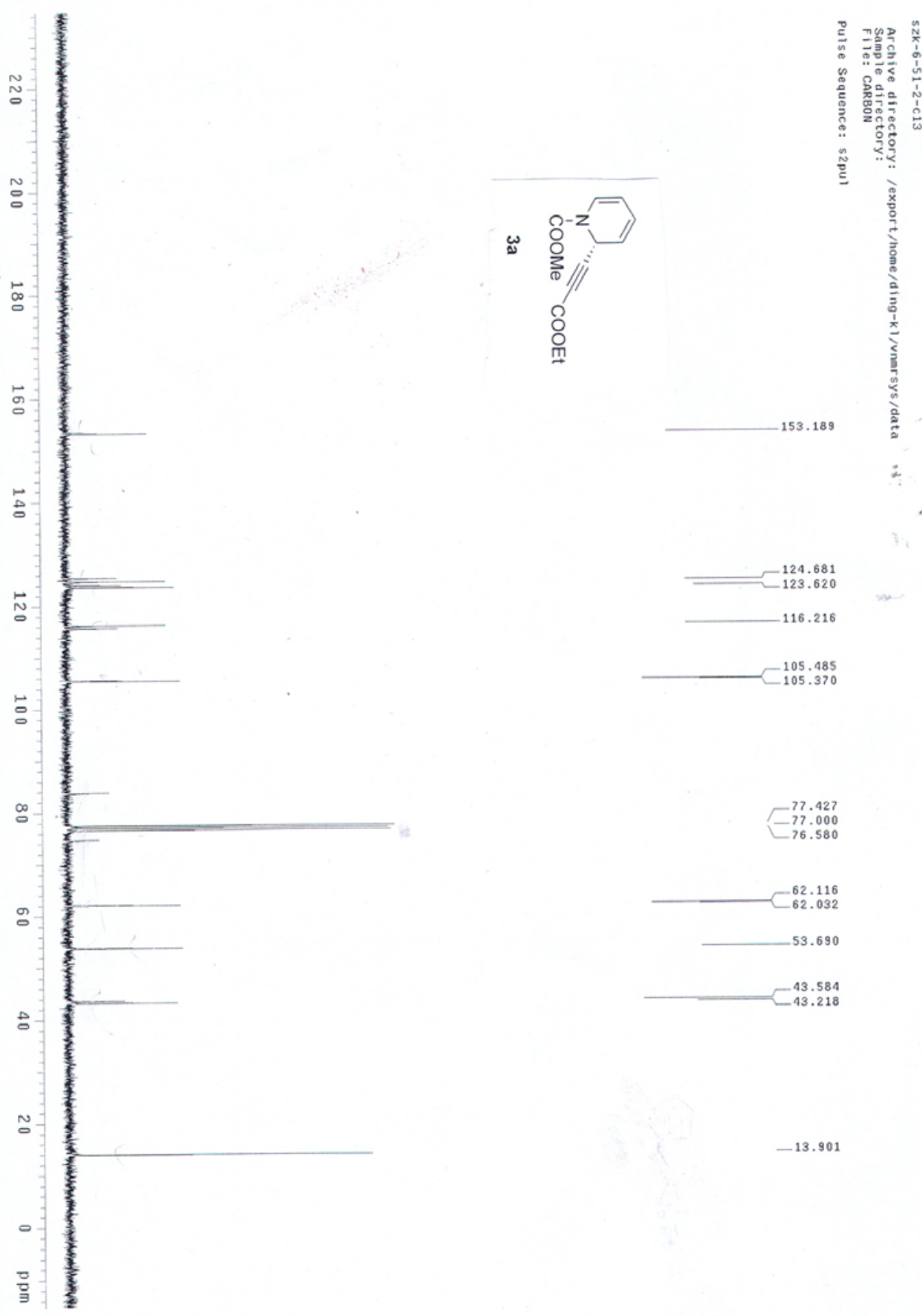

$-13.901$ 
Software Version: 4.1<2F12>

Date: 06-9-21 10:01

Sample Name : $5-95-1-1+1-$

Data File : D: ICS $\backslash D-2$ 499.RAW Date: 06-9-21 9:31

Sequence File: D: \CS\D-2.SEQ Cycle: 1 Channe1 : A

Instrument : $970 \mathrm{~A}_{-}-0$ Rack/Vial: $0 / 0$ Operator: DING

Sample Amount $: \overline{1} .0000 \quad$ Dilution Factor $: 1.00$

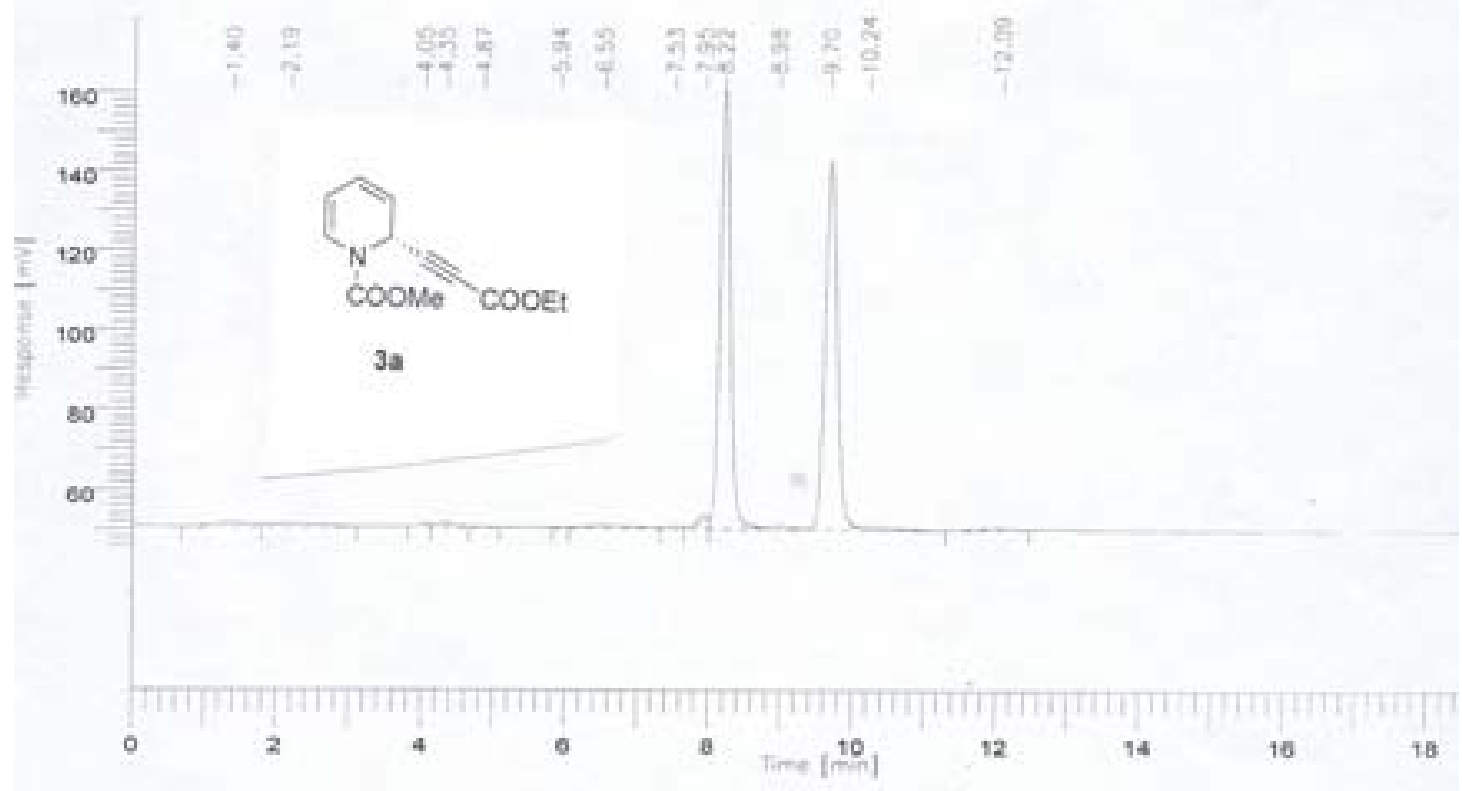

DEFAULT REPORT

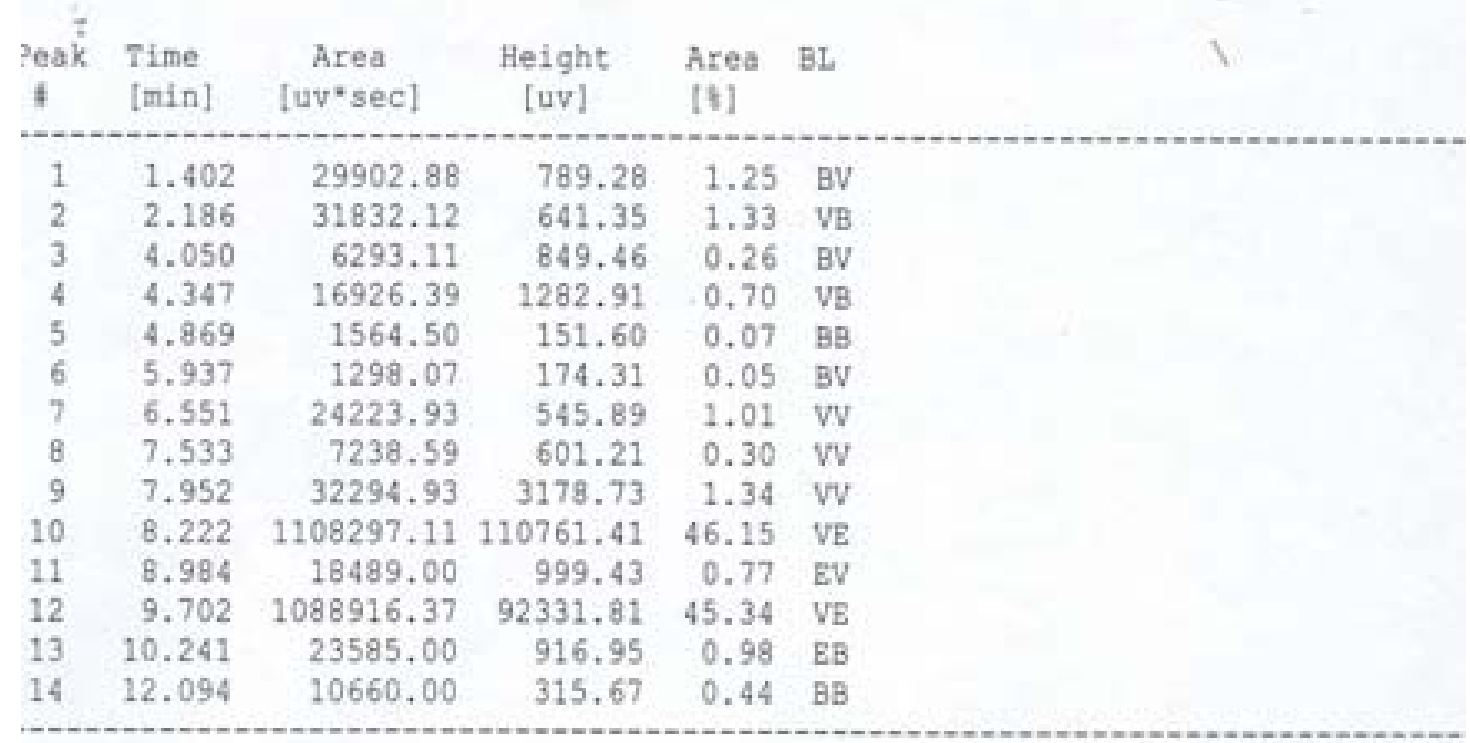


Software Version: $4,1<2 \mathrm{~F} 12\rangle$

Date: 06-9-21 10:09

Sanple Name : 5-95-1-2

Data File : D: \CS\D-2.500.RAK Date: 06-9-21 9:52

Sequence File: D: $\backslash$ CS $\backslash D-2 . S B Q$ Cyelet 1 Channel : A

Instrument : $970 \mathrm{~A}-0$ Rack/Vial: $0 / 0$ Operator: DING

Sample Amount : $\overline{1} . \overline{0000}$ Dilution Factor $: 1.00$

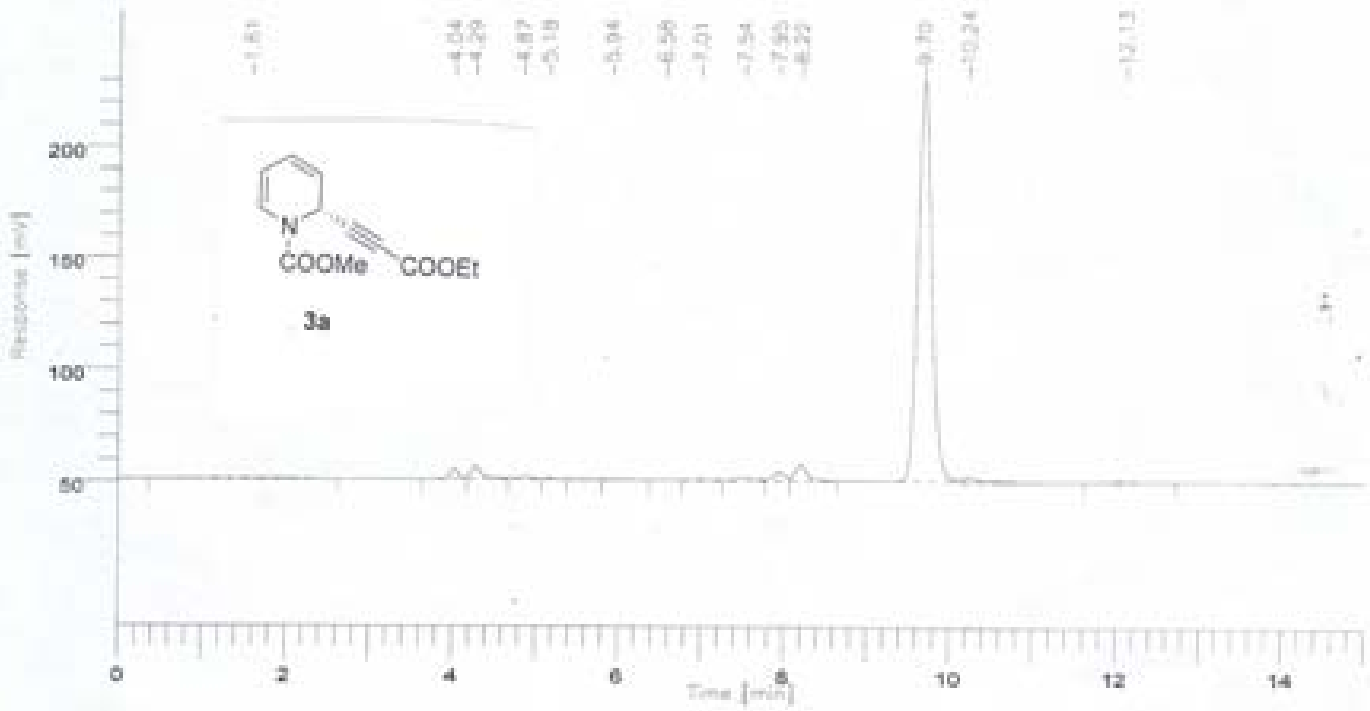

DEFAULT REPORT

\begin{tabular}{|c|c|c|c|c|c|c|}
\hline Peak & $\begin{array}{l}\text { Time } \\
\text { [min] }\end{array}$ & $\begin{array}{c}\text { Area } \\
\text { [uv'sec] }\end{array}$ & $\begin{array}{l}\text { Height } \\
\text { [uv] }\end{array}$ & $\begin{array}{l}\text { Area } \\
{[8]}\end{array}$ & BL & \\
\hline 1 & 1.608 & 25405.00 & 331.94 & 1.05 & BB & \\
\hline 2 & 4.041 & 31696.32 & 4811.97 & 1.31 & BV & \\
\hline 3 & 4.290 & 54577.57 & 6038.93 & 2.26 & VV & \\
\hline 4 & 4.865 & 12310.69 & 1205.45 & 0.51 & VV & \\
\hline 5 & 5.175 & 4372.42 & 599.40 & 0.18 & $\mathrm{VB}$ & \\
\hline 6 & 5.935 & 2000.00 & 188.17 & 0.08 & $\mathrm{BB}$ & \\
\hline 7 & 6.581 & 1045.00 & 97.96 & 0.04 & BB & 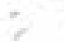 \\
\hline 8 & 7.005 & 2747.38 & 252.42 & 0.11 & BV & \\
\hline 9 & 7.536 & 16604.70 & 1500.93 & 0.69 & VV & \\
\hline 10 & 7.954 & 37485.88 & 3987.49 & 1.55 & VV & \\
\hline 11 & 8.220 & 71631.54 & 7239.41 & 2.97 & $\mathrm{VB}$ & \\
\hline 12 & 9.697 & 2108857.00 & 180670.77 & 87.43 & $\mathrm{BE}$ & \\
\hline 13 & 10.236 & 36076.00 & 2177.26 & 1.50 & $\mathrm{~EB}$ & \\
\hline 14 & 12.125 & 7374.00 & 351.90 & 0.31 & $\mathrm{BB}$ & \\
\hline
\end{tabular}




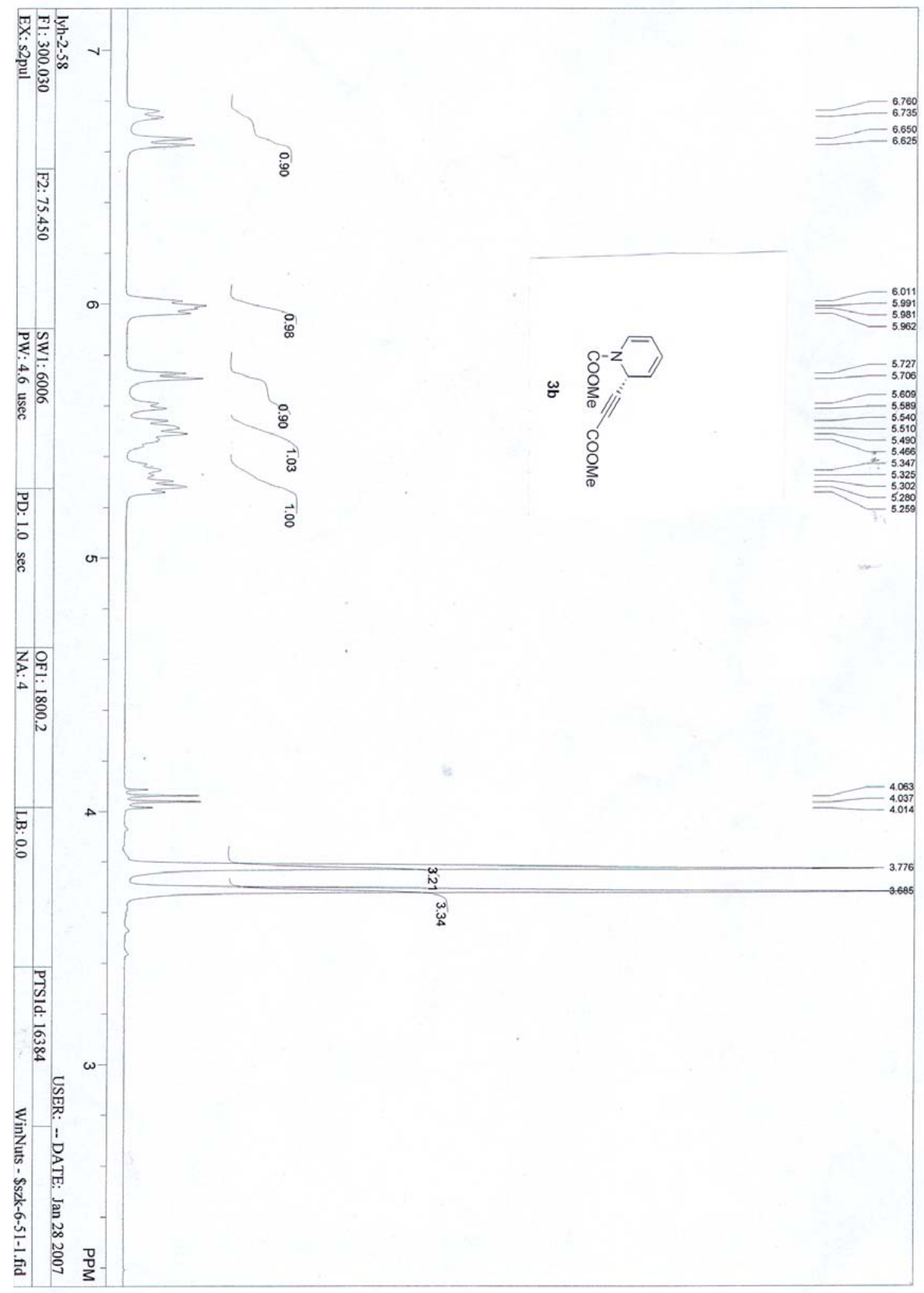



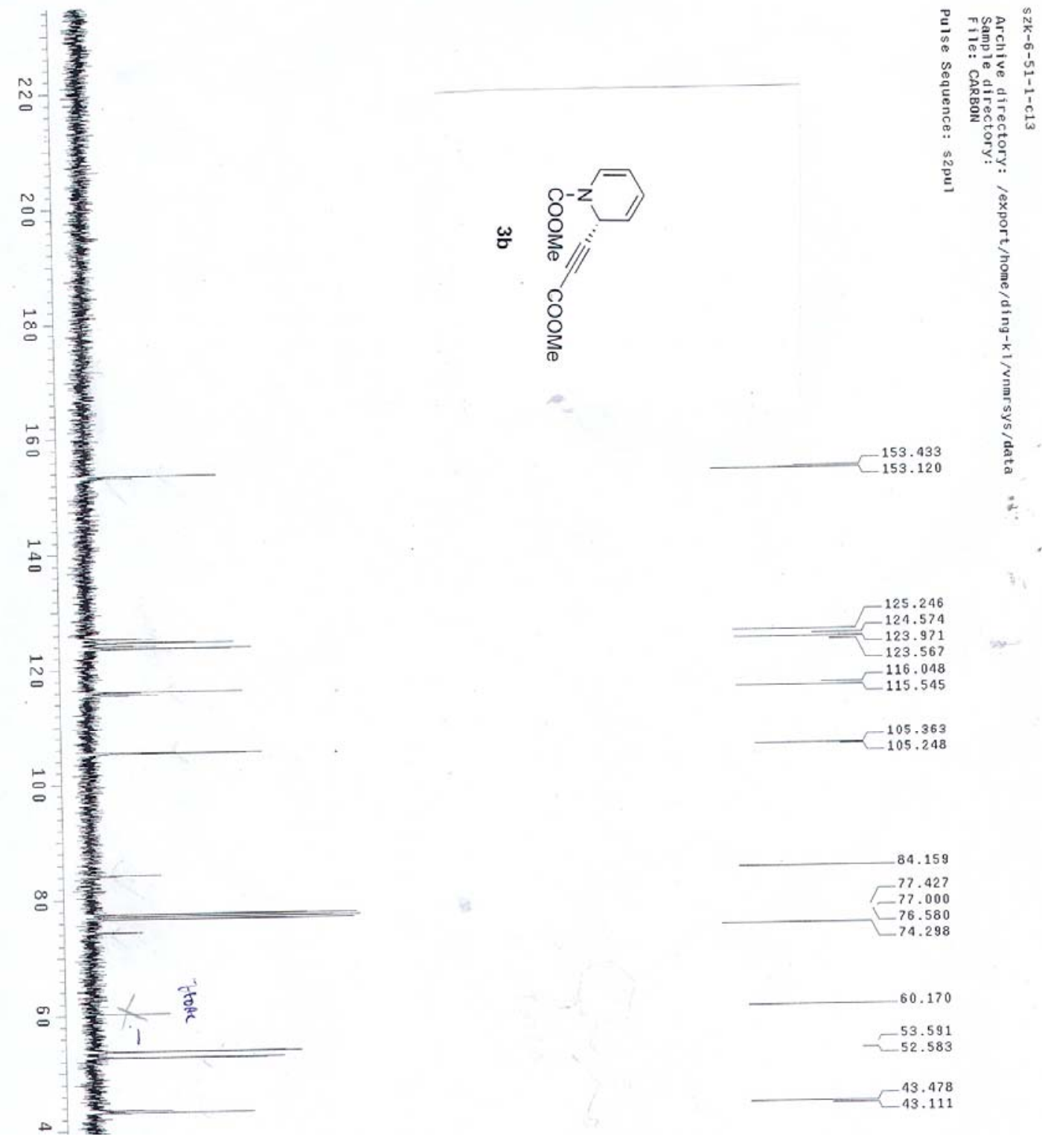


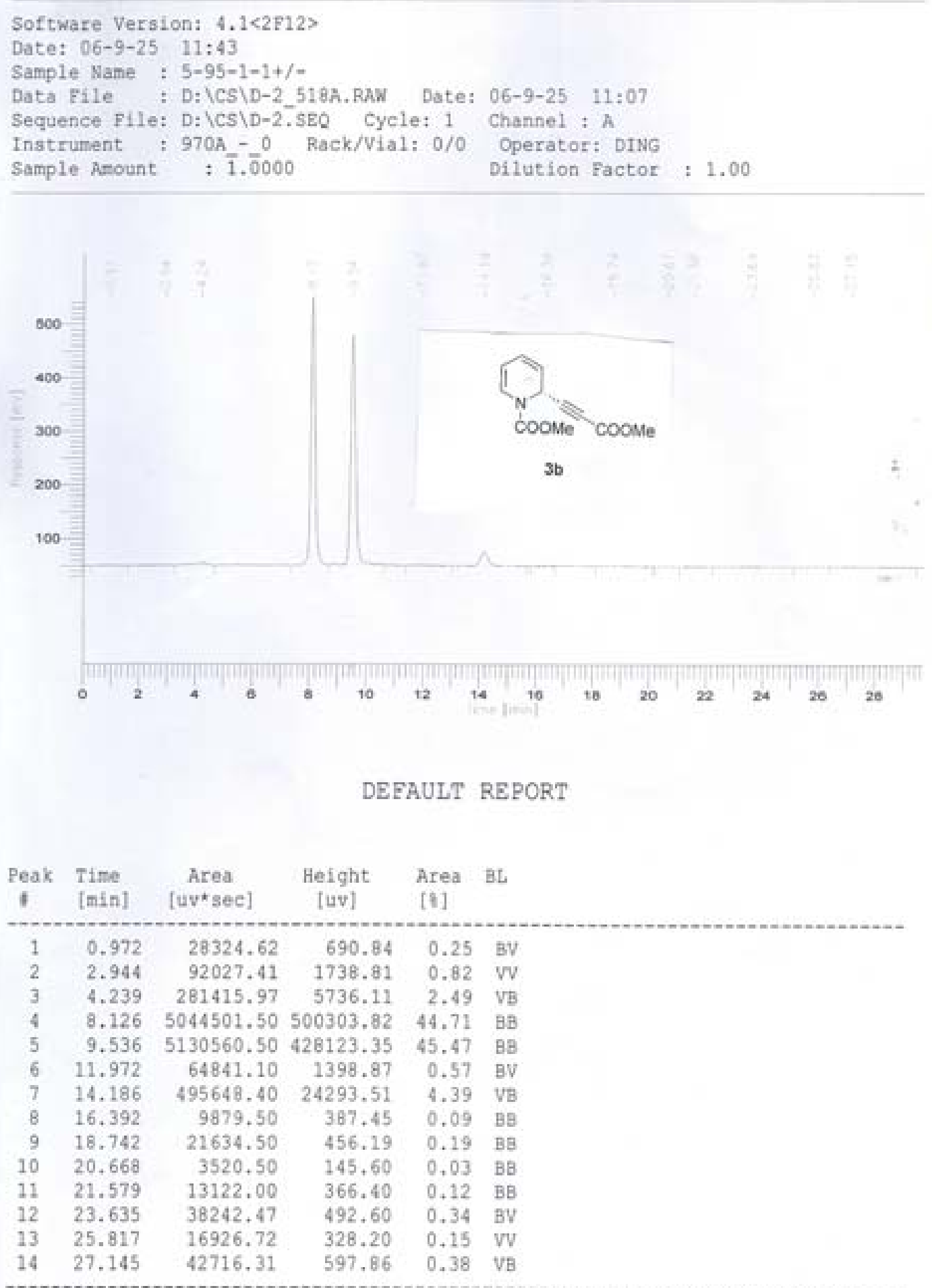




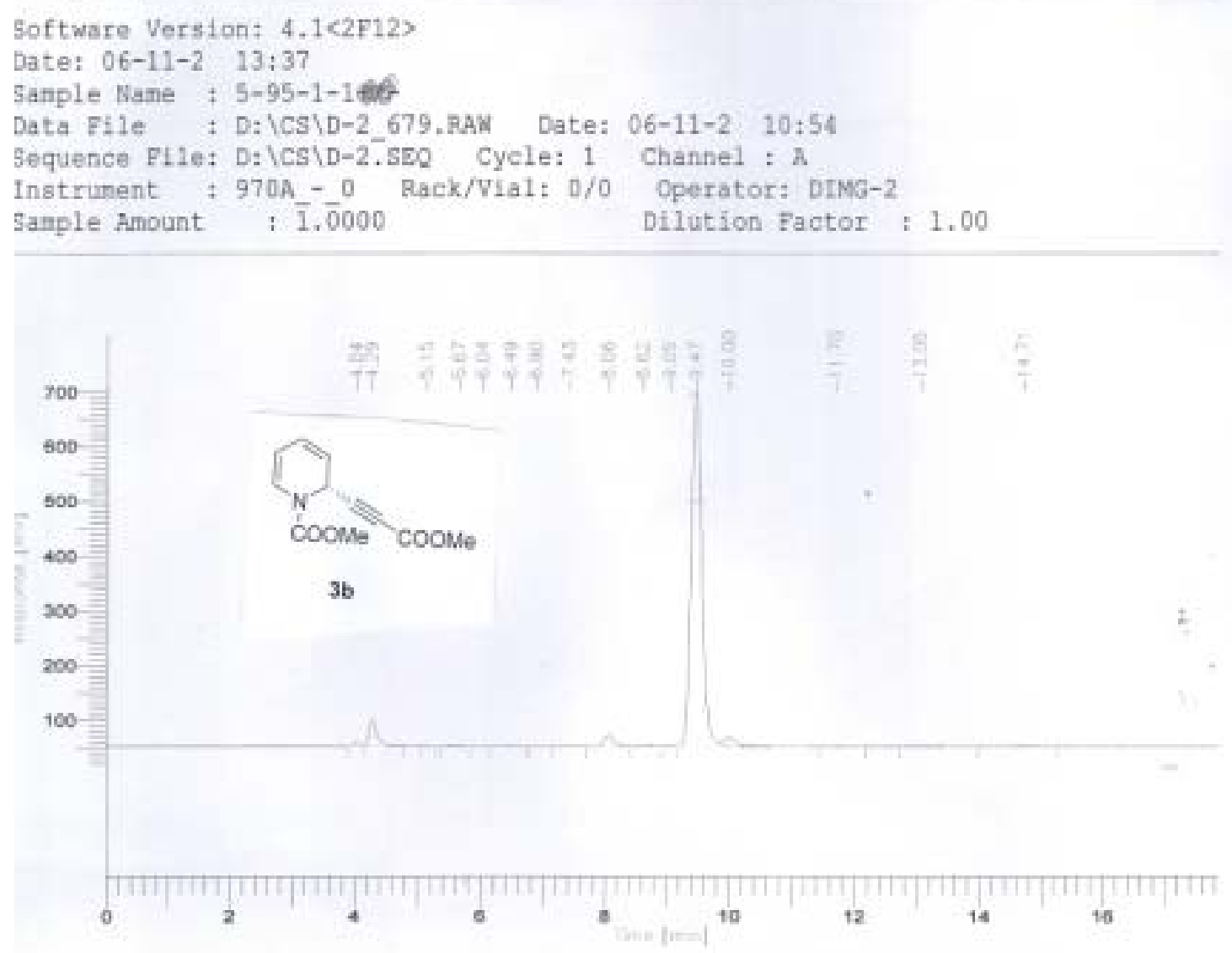

DEFAULT REPORT

\begin{tabular}{|c|c|c|c|c|c|}
\hline $\begin{array}{l}\text { leak } \\
\text { t }\end{array}$ & $\begin{array}{l}\text { Tine } \\
\text { [nin] }\end{array}$ & $\begin{array}{c}\text { Area } \\
\text { [uv*gec] }\end{array}$ & $\begin{array}{c}\text { Height } \\
\text { [uv] }\end{array}$ & $\begin{array}{l}\text { Area } \\
\text { [B] }\end{array}$ & BL \\
\hline 1 & 4.040 & 57587.99 & 9270.38 & 0.67 & BV \\
\hline 2 & 4.285 & 424428.51 & 50063.77 & 4.95 & VB \\
\hline 3 & 5.153 & 2597,00 & 480.58 & 0.03 & $\mathrm{BB}$ \\
\hline 4 & 5.665 & 10710.89 & 800.24 & 0.12 & BV \\
\hline 5 & 6.042 & 1571.78 & 156.07 & 0.02 & vV \\
\hline 6 & 6.491 & 8293.84 & 756.85 & 0.10 & $\mathrm{VB}$ \\
\hline 7 & 6.900 & 3587.53 & 239.35 & 0.04 & BV \\
\hline 8 & 7.428 & 6573.35 & 486.60 & 0.08 & vV \\
\hline 9 & 8.063 & 206467.07 & 20788.09 & 2.41 & WV \\
\hline 10 & 8.617 & 30292.33 & 2403.49 & 0.35 & wV \\
\hline 11 & 9.049 & 5784.92 & 569.34 & 0.07 & vV \\
\hline 12 & 9.467 & 7586068.80 & 649947.25 & 88.49 & VE \\
\hline 13 & 9.996 & 200395.00 & 15580.59 & 2.34 & EA \\
\hline 14 & 11.704 & 2350.00 & 126.61 & 0.03 & BA \\
\hline 15 & 13.054 & 16763.22 & 739.98 & 0.20 & BV \\
\hline
\end{tabular}




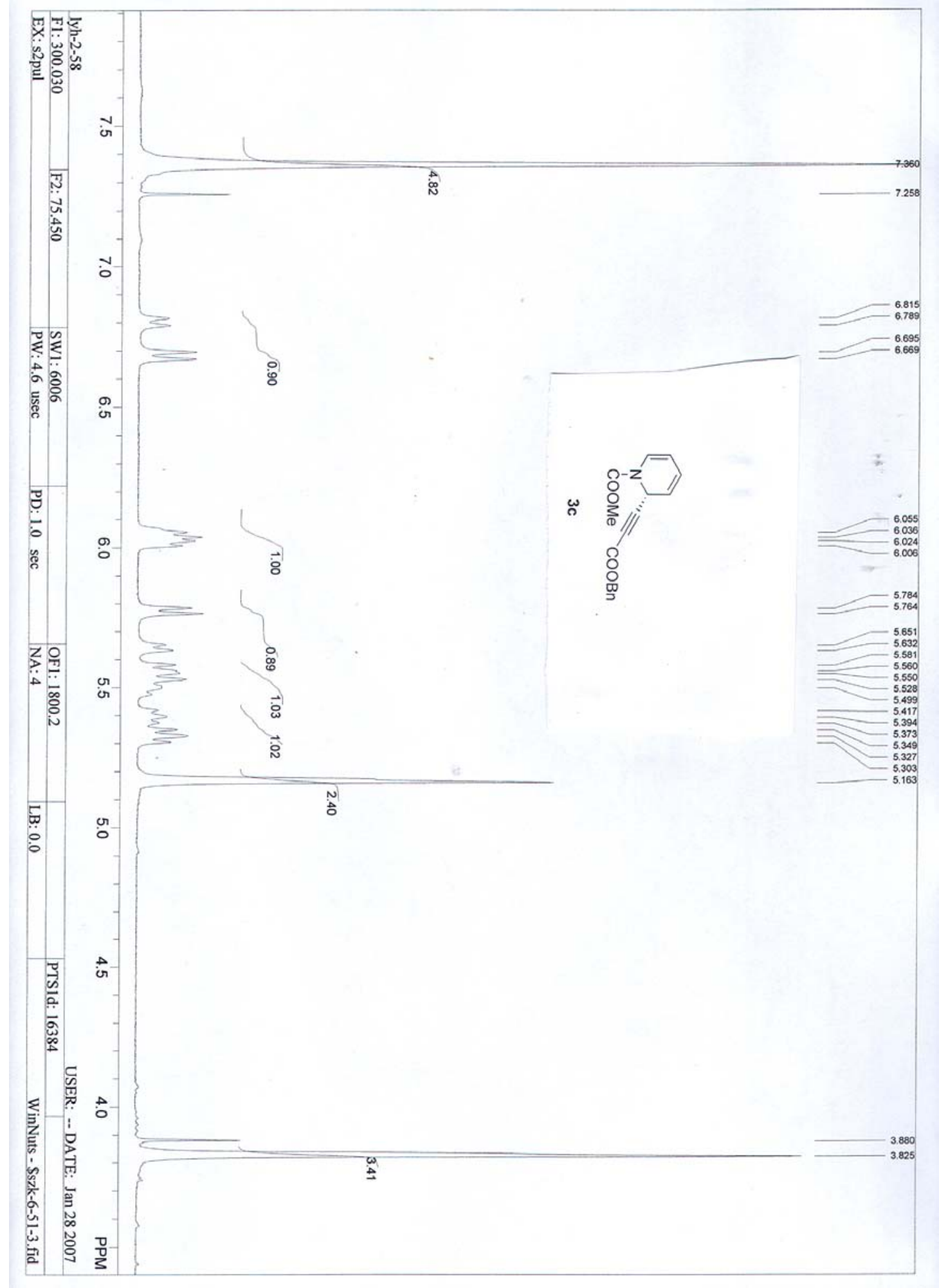




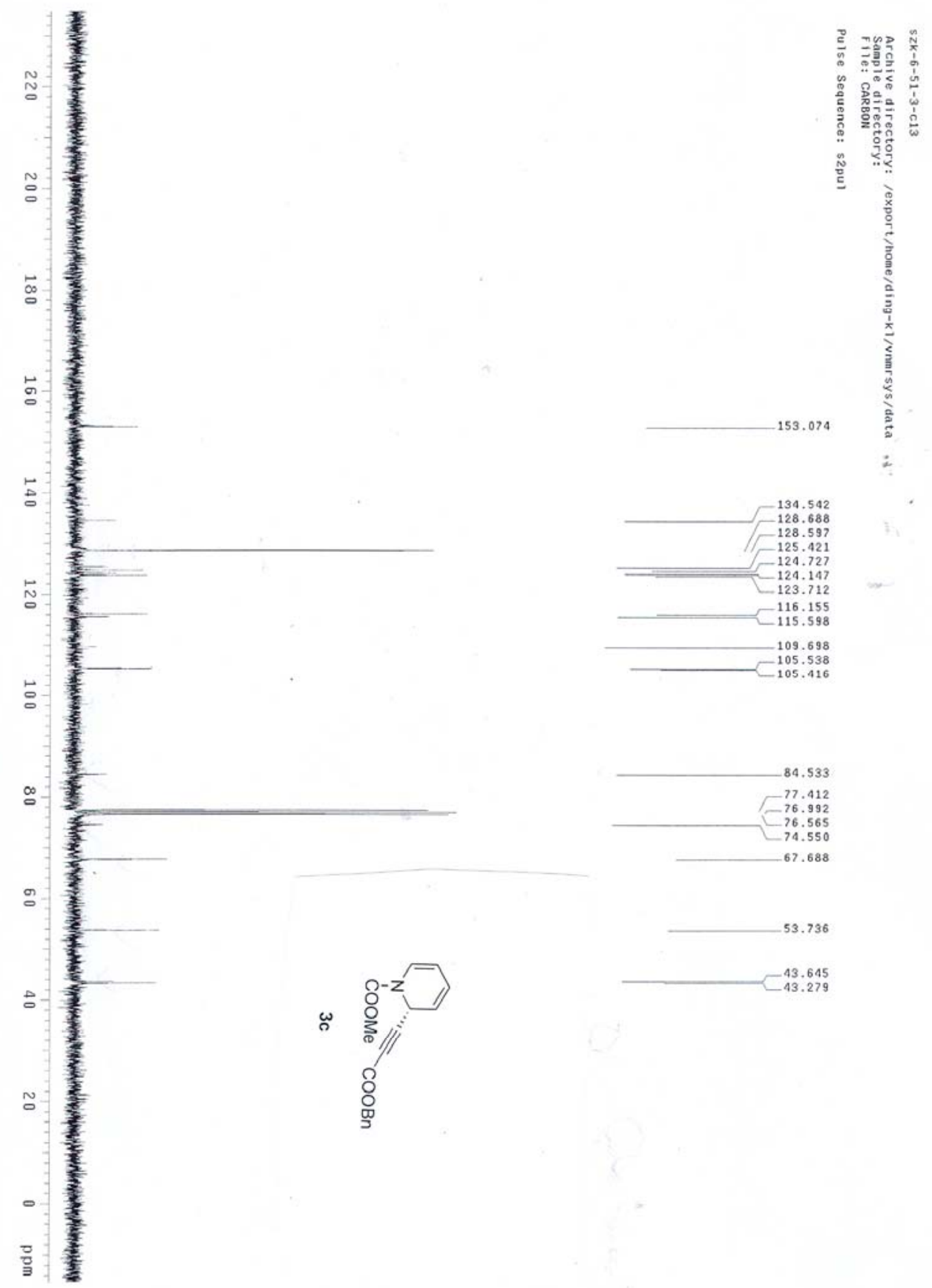


Software Version: 4.1<2F12>

Date: 06-11-9 14:29

Sample Name : 6-17-1-1+/-

Data File : D: \CS \D-2_718. RAW Date: 06-11-9 13:48

Sequence File: D: \CS \D-2.SEQ Cycle: 1 Channel : A

Instrument : 970A-0 Rack/Vial: 0/0 Operator: DIMG-2

Sample Amount : $\overline{1} . \overline{0} 000 \quad$ Dilution Factor : 1.00

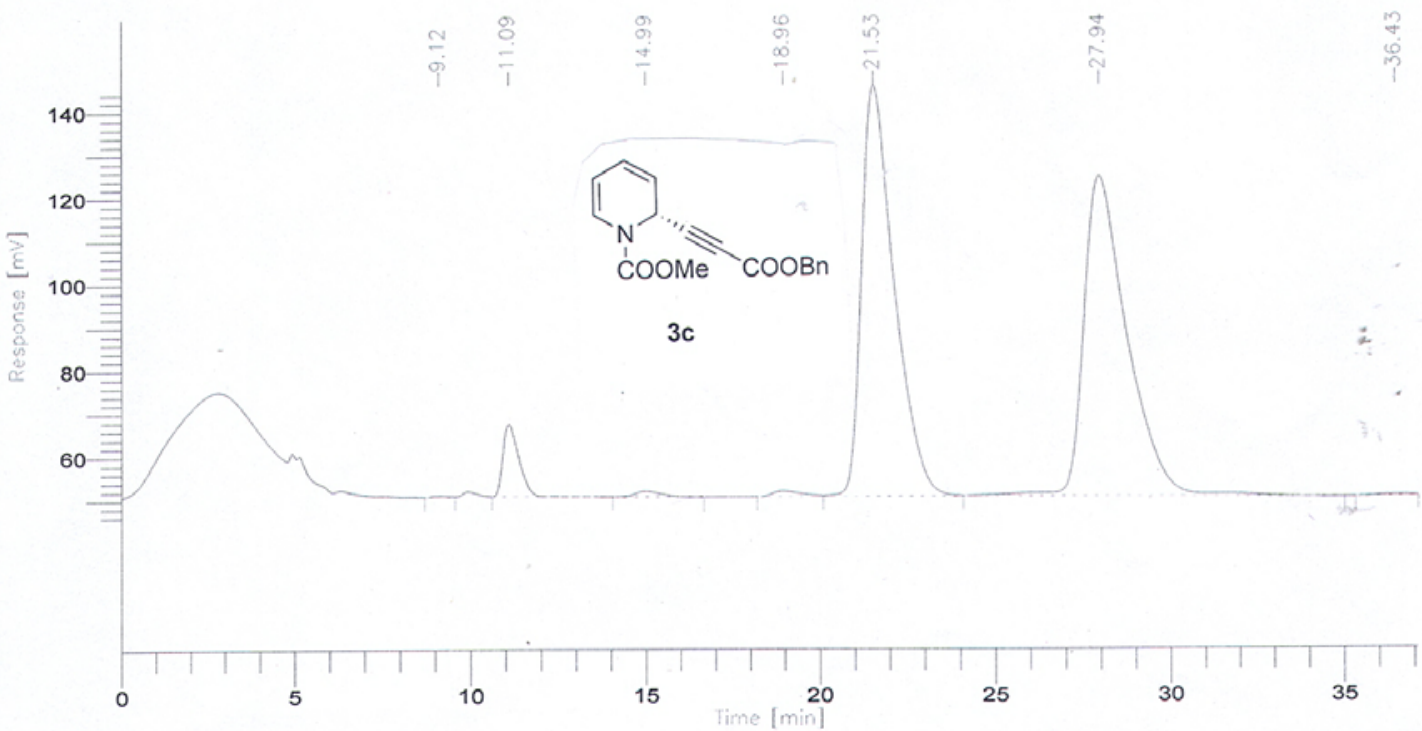

DEFAULT REPORT

\begin{tabular}{|c|c|c|c|c|c|}
\hline $\begin{array}{c}\text { Peak } \\
\#\end{array}$ & $\begin{array}{l}\text { Time } \\
\text { [min] }\end{array}$ & $\begin{array}{c}\text { Area } \\
{\left[u v^{*} s e c\right]}\end{array}$ & $\begin{array}{l}\text { Height } \\
\text { [uv] }\end{array}$ & $\begin{array}{l}\text { Area } \\
{[\%]}\end{array}$ & BL \\
\hline 1 & 9.119 & 5524.00 & 221.59 & 0.04 & $\mathrm{BB}$ \\
\hline 2 & 11.094 & 525721.00 & 16722.98 & 3.99 & $\mathrm{BB}$ \\
\hline 3 & 14.992 & 66057.50 & 1329.69 & 0.50 & $\mathrm{BB}$ \\
\hline 4 & 18.955 & 72919.52 & 1309.21 & 0.55 & BV \\
\hline 5 & 21.529 & 6136375.48 & 95260.31 & 46.54 & VB \\
\hline 6 & 27.943 & 6366450.00 & 74191.32 & 48.28 & BB \\
\hline 7 & 36.431 & 12225.00 & 204.28 & 0.09 & $\mathrm{BB}$ \\
\hline
\end{tabular}

13185272.50189239 .39100 .00 


\section{色谱分析报告}

样品名称:

样品批号:

分析日期: 2006-04-26
样品文件名:4-79-1-02-1. che

分析者:

分析时间: $13: 50$

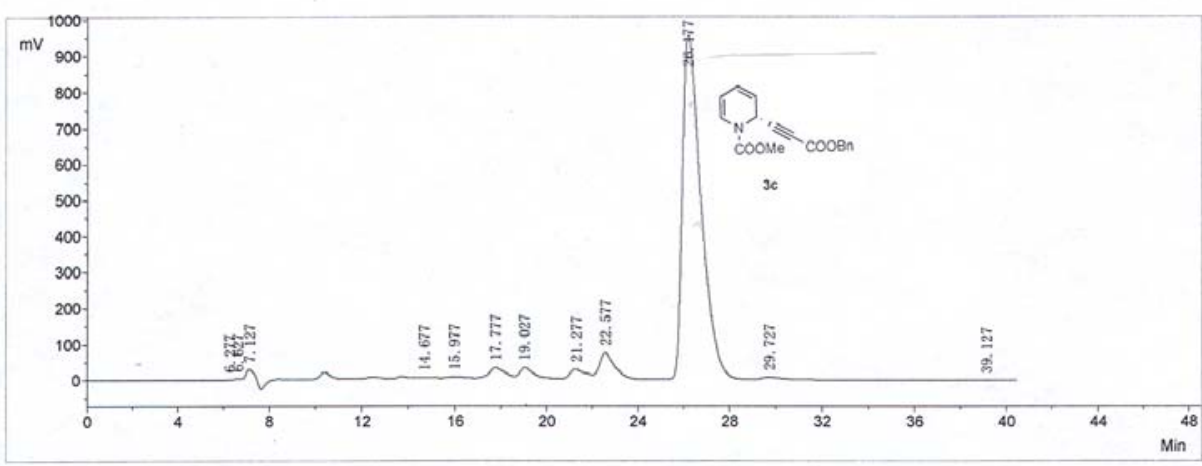

\begin{tabular}{|c|c|c|c|c|c|c|}
\hline 序号 & 峰号 & 组份名 & 保留时间 & 峰高 & 峰面积 & 面积百分比 (\%) \\
\hline 1 & 1 & & 6.277 & 15823.1 & 892966.8 & 1. 3664 \\
\hline 2 & 2 & & 6.627 & 21595.1 & 419097.1 & 0.6413 \\
\hline 3 & 3 & & 7. 127 & 51875.1 & 1753284.1 & 2. 6829 \\
\hline 4 & 4 & & 14. 677 & 1212.5 & 42197.2 & 0.0646 \\
\hline 5 & 5 & & 15. 977 & 3852.3 & 182076.0 & 0.2786 \\
\hline 6 & 6 & & 17. 777 & 31239.3 & 1446047.4 & 2. 2127 \\
\hline 7 & 7 & & 19. 027 & 31573.7 & 1311551.7 & 2. 0069 \\
\hline 8 & 8 & & 21.277 & 27831.8 & 1310899.1 & 2. 0059 \\
\hline 9 & 9 & & 22.577 & 73275.9 & 3412993.8 & 5. 2226 \\
\hline 10 & 10 & & 26. 177 & 955359.6 & 54200758.7 & 82.9382 \\
\hline 11 & 11 & & 29.727 & 5942.2 & 329585.3 & 0.5043 \\
\hline 12 & 12 & & 39.127 & 609.5 & 49348.1 & 0.0755 \\
\hline
\end{tabular}




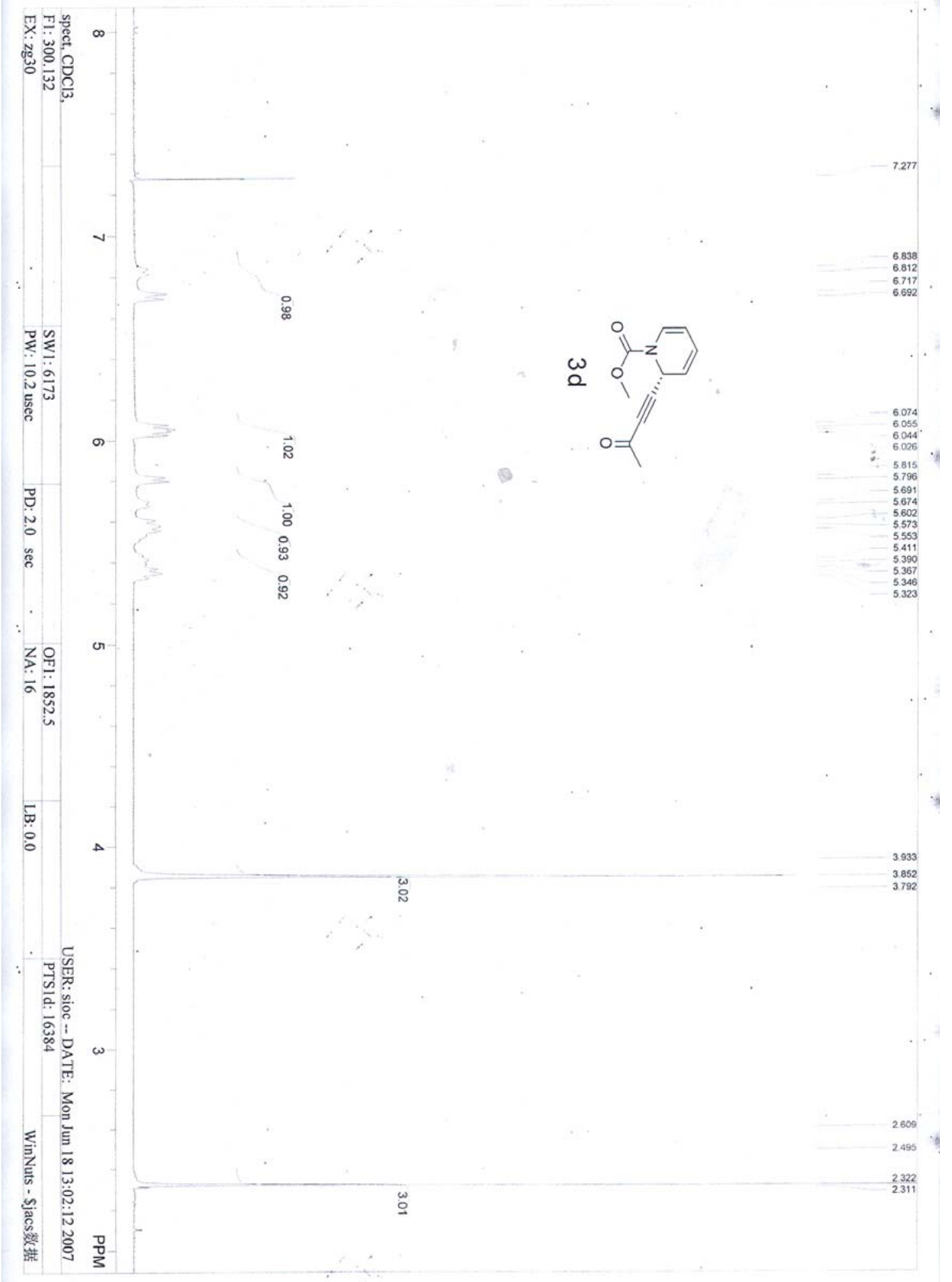




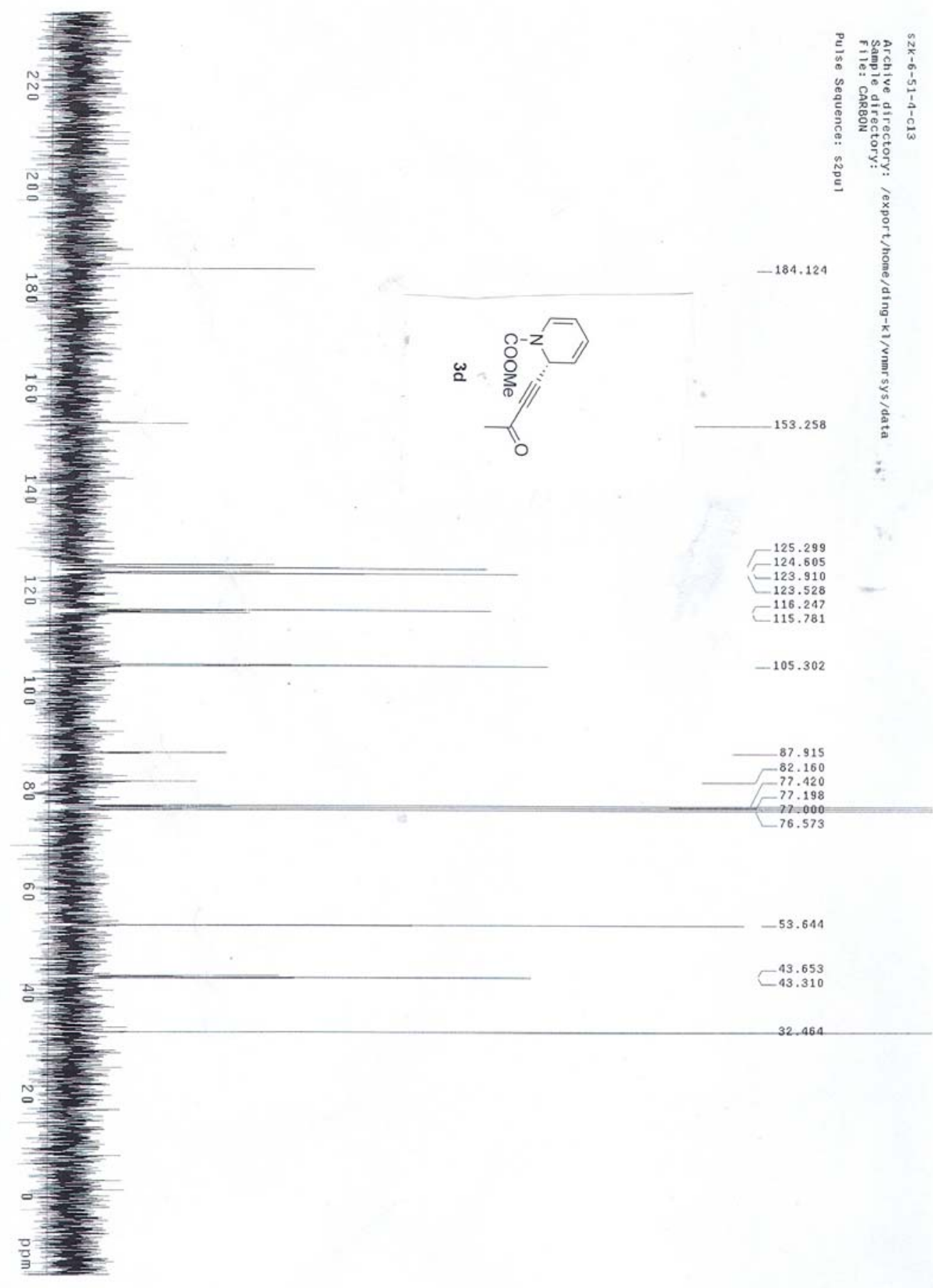




\section{色谱分析报告}

\section{样品名称:}

样品批号:

分析日期: 2006-12-06

样品文件名:6-21-1+-. che

分析者:

分析时间: $13: 31$
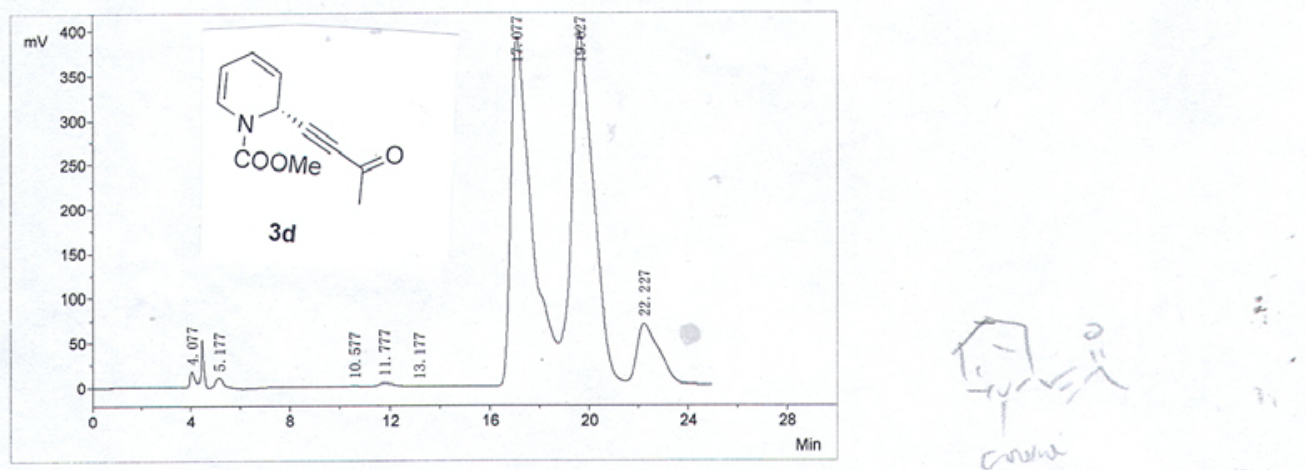

\begin{tabular}{|c|c|c|c|c|c|c|}
\hline 序号 & 峰号 & 组份名 & 保留时间 & 峰高 & 峰面积 & 面积百分比 (\%) \\
\hline 1 & 1 & & 4. 077 & 17247.1 & 247061.8 & 0.4593 \\
\hline 2 & 2 & & 5. 177 & 11101.5 & 649438.9 & 1. 2074 \\
\hline 3 & 3 & & 10.577 & 1241.6 & 279118.7 & 0.5189 \\
\hline 4 & 4 & & 11.777 & 4079.4 & 186872.2 & 0.3474 \\
\hline 5 & 5 & & 13. 177 & 149.3 & 4729.4 & 0.0088 \\
\hline 6 & 6 & & 17.077 & 390972.1 & 22964785.7 & 42.6931 \\
\hline 7 & 7 & & 19. 627 & 398132.6 & 24899566.1 & 46. 2900 \\
\hline 8 & 8 & & 22.227 & 69438.3 & 4558777.3 & 8.4751 \\
\hline 合计: & & & & 892361.8 & 53790350.2 & 100.0000 \\
\hline
\end{tabular}




\section{色谱分析报告}

样品名称：

样品文件名:6-271-2. che

样品批号:

分析者:

分析日期:2006-12-06

分析时间: $14: 36$

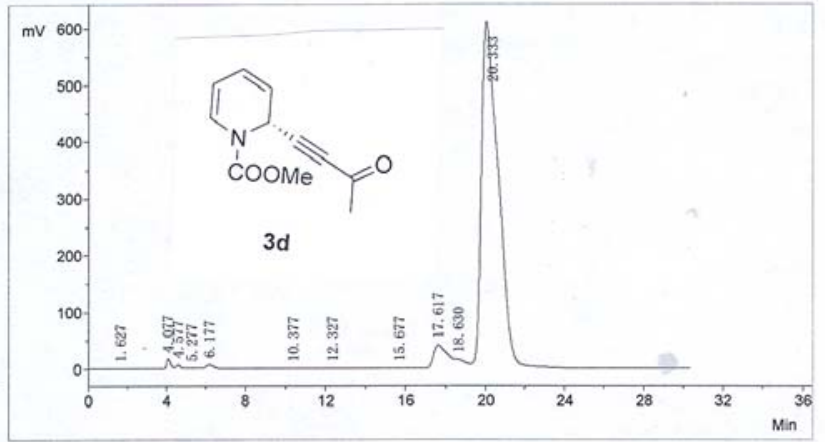

\begin{tabular}{|c|c|c|c|c|c|c|}
\hline 序号 & 峰号 & 组份名 & 保留时间 & 峰高 & 峰面积 & 面积百分比 (\%) \\
\hline 1 & 1 & & 1.627 & 557.2 & 46837.6 & 0.1274 \\
\hline 2 & 2 & & 4. 077 & 17272.0 & 255244.3 & 0.6945 \\
\hline 3 & 3 & & 4. 577 & 6962.1 & 118726.8 & 0.3231 \\
\hline 4 & 4 & & 5.277 & 1823.3 & 53344.0 & 0.1451 \\
\hline 5 & 5 & & 6.177 & 6813.4 & 196045.9 & 0.5334 \\
\hline 6 & 6 & & 10.377 & 580.5 & 17846.2 & 0.0486 \\
\hline 7 & 7 & & 12.327 & 308.4 & 10922.8 & 0.0297 \\
\hline 8 & 8 & & 15.677 & 198.0 & 9276.6 & 0.0252 \\
\hline 9 & 9 & & 17.617 & 34690.7 & 1324520.4 & 3. 6040 \\
\hline 10 & 10 & & 18.630 & 1686.9 & 32844.0 & 0.0894 \\
\hline 11 & 11 & & 20.333 & 484999.4 & 34685605.6 & 94. 3795 \\
\hline
\end{tabular}




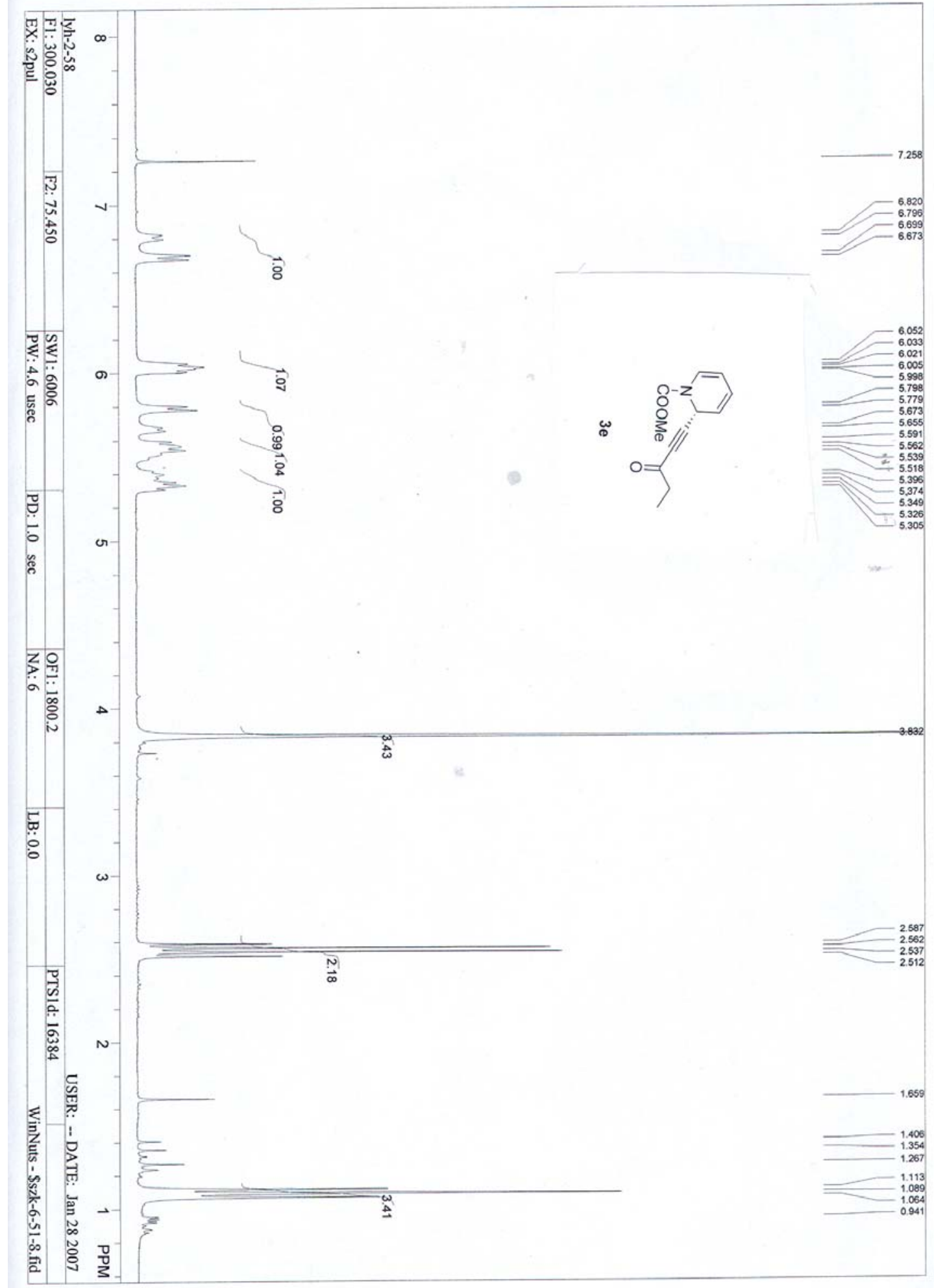




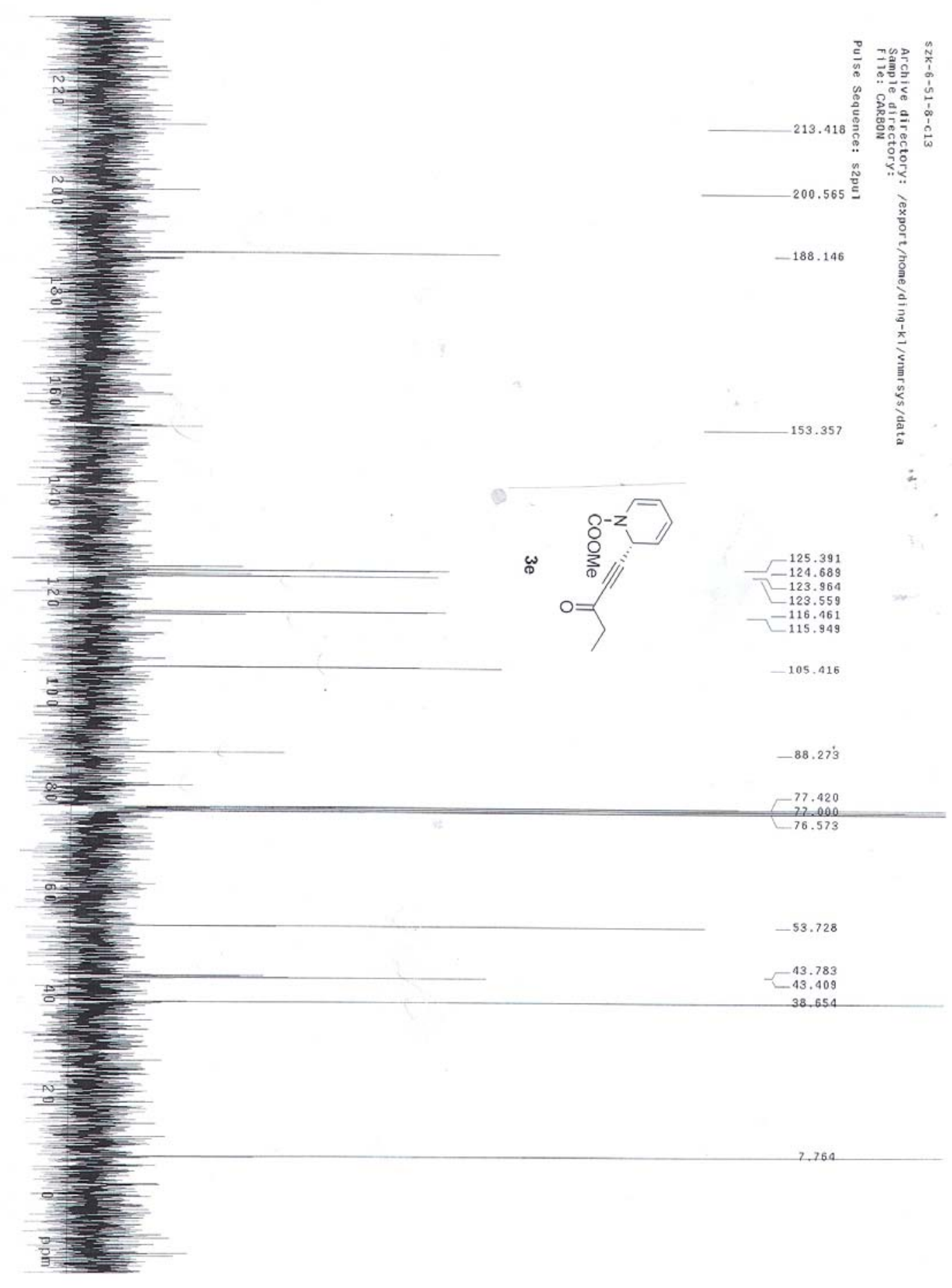




\section{色谱分析报告}

样品名称:

样品批号:

分析日期: 2006-12-13

样品文件名:6-18-2-1+-. che

分析者:

分析时间: $08: 42$

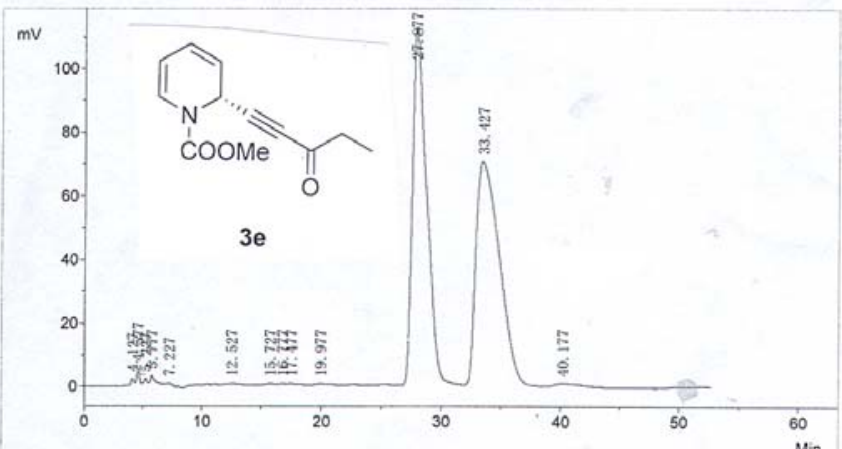

Min

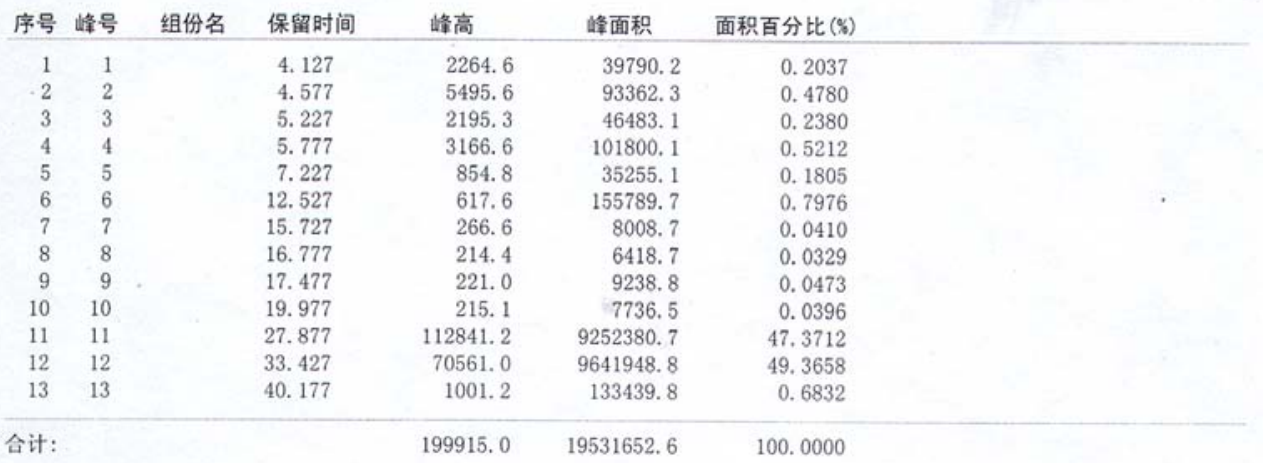




\section{色谱分析报告}

样品名称:

样品批号:

分析日期: 2006-12-13
样品文件名:6-18-2-2. che

分析者:

分析时间: 09:40

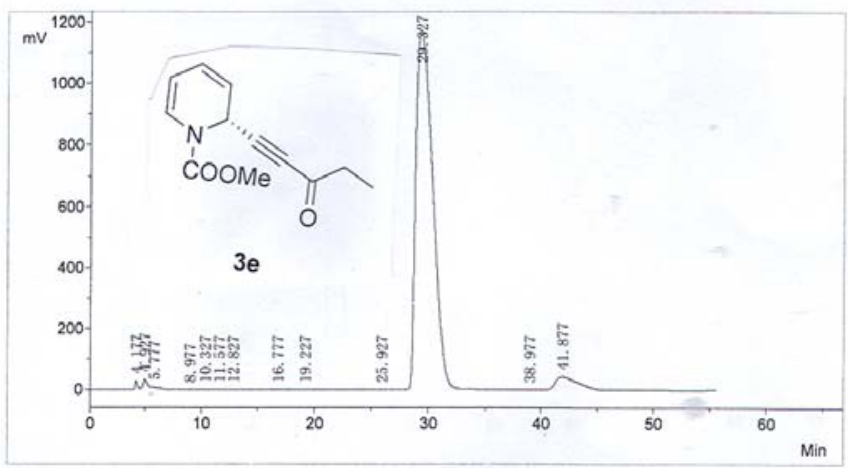

\begin{tabular}{|c|c|c|c|c|c|c|}
\hline 序号 & 峰号 & 组份名 & 保留时间 & 峰高 & 峰面积 & 面积百分比 (\%) \\
\hline 1 & 1 & & 4. 177 & 28432.2 & 415279.2 & 0.3217 \\
\hline 2 & 2 & & 4.927 & 33520.2 & 978970.5 & 0.7583 \\
\hline 3 & 3 & & 5. 777 & 7952.9 & 317095.7 & 0.2456 \\
\hline 4 & 5 & & 10. 327 & 152.8 & 5453.8 & 0.0042 \\
\hline 5 & 6 & & 11.577 & 248.0 & 10057.0 & 0.0078 \\
\hline 6 & 7 & & 12. 827 & 267.8 & 9004.1 & 0.0070 \\
\hline 7 & 8 & & 16. 777 & 1051.7 & 69061.1 & 0.0535 \\
\hline 8 & 9 & & 19. 227 & 1066.4 & 52874.1 & 0.0410 \\
\hline 9 & 10 & & 25.927 & 201.8 & 47117.2 & 0.0365 \\
\hline 10 & 11 & & 29. 327 & 1174498.3 & 121232472.9 & 93.9069 \\
\hline 11 & 12 & & 38.977 & 231.9 & 31717.9 & 0.0246 \\
\hline 12 & 13 & & 41.877 & 42616.2 & 5929430.9 & 4. 5929 \\
\hline 1. & & & & 1290240.0 & 129098534.5 & 100.0000 \\
\hline
\end{tabular}




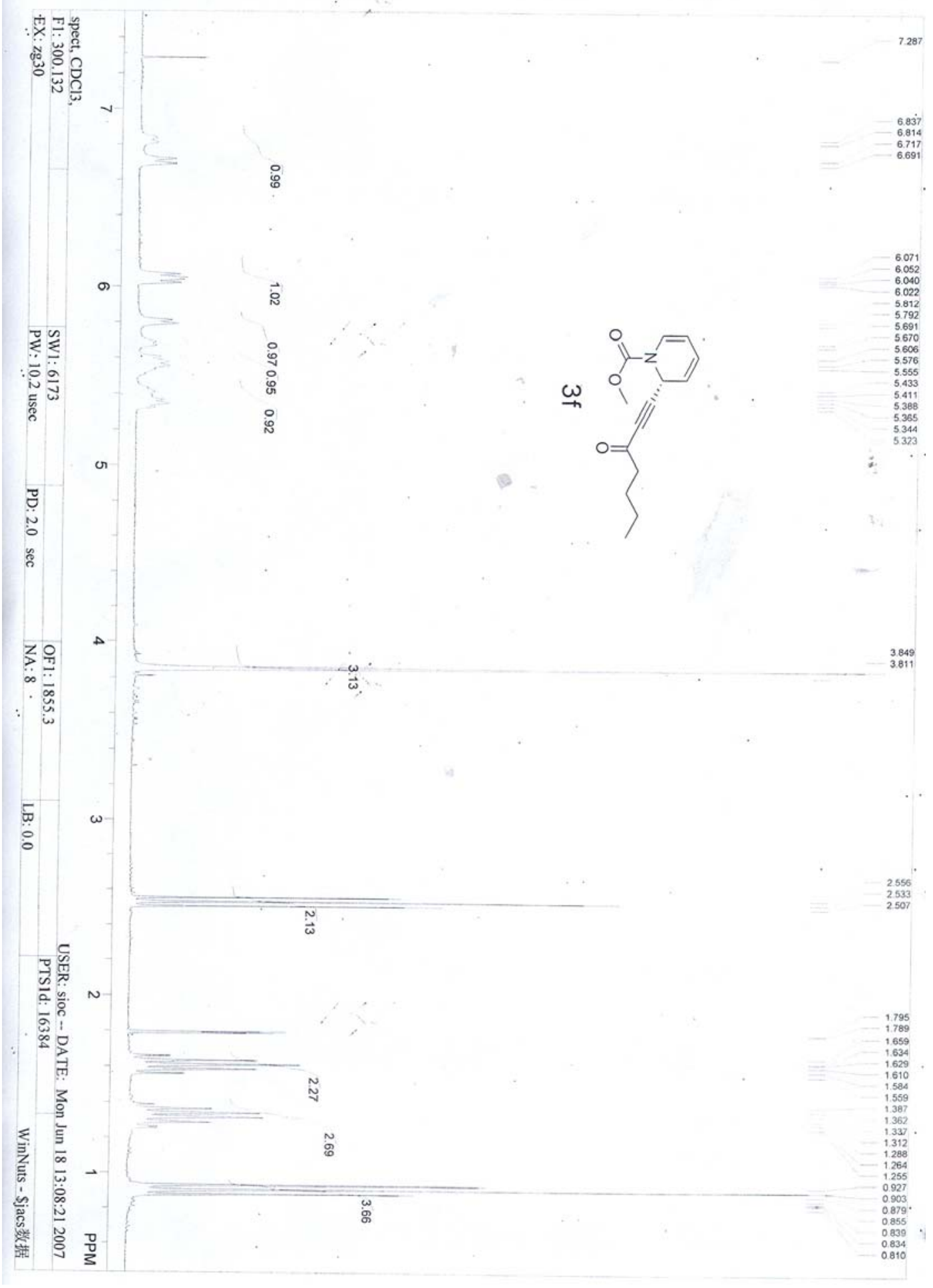




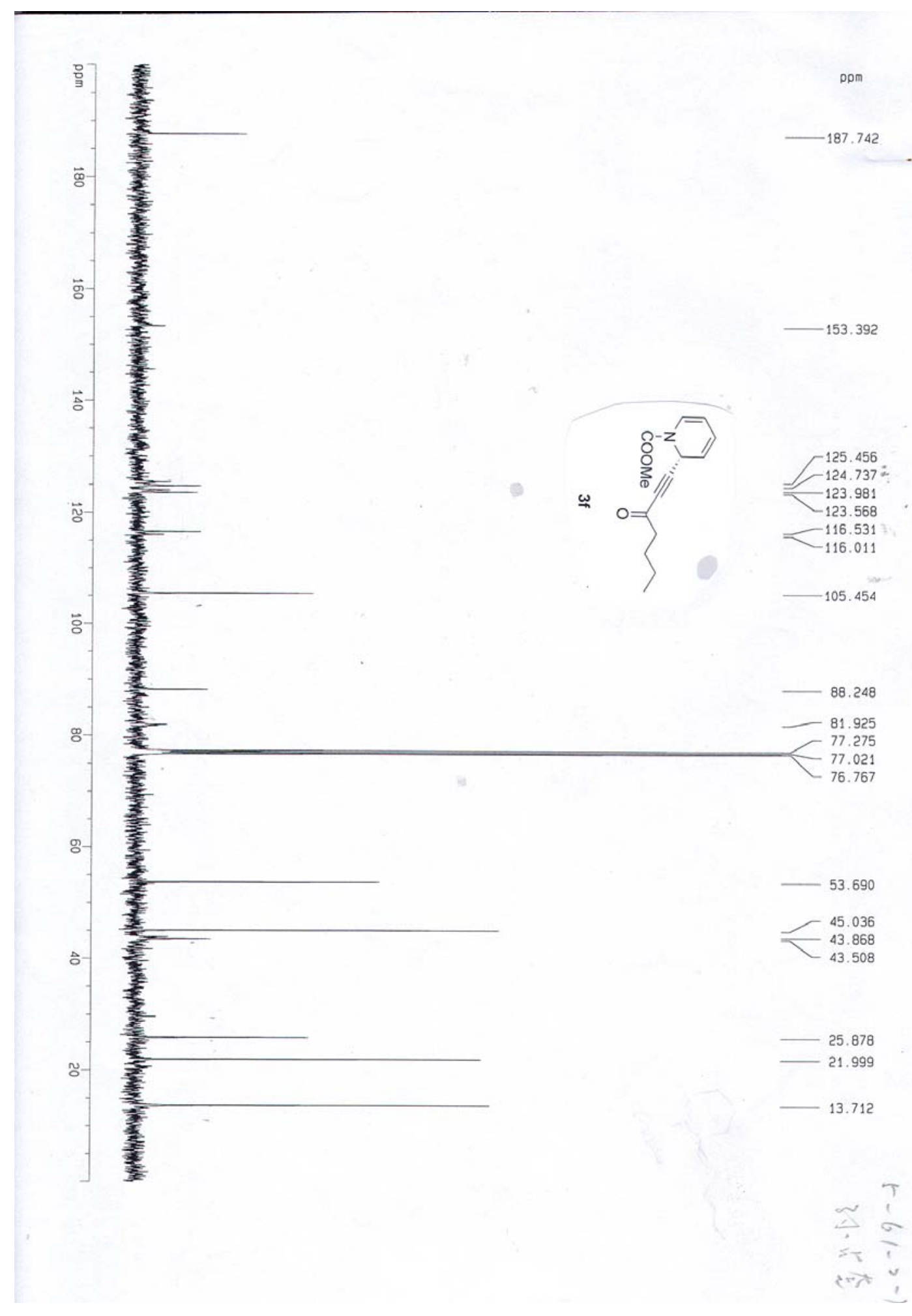


Software Version: $4.1<2 \mathrm{~F} 12\rangle$

Date: 06-11-9 10:49

Sample Name : 5-61-2-1+/-

Data File : D: \CS \D-2 714.RAW Date: 06-11-9 10:29

Sequence File: D: \CS $\backslash D-2$.SEQ Cycle: 1 Channel : A

Instrument : 970A - 0 Rack/Vial: 0/0 Operator: DIMG-2

Sample Amount : $\overline{1} . \overline{0} 000 \quad$ Dilution Factor $: 1.00$

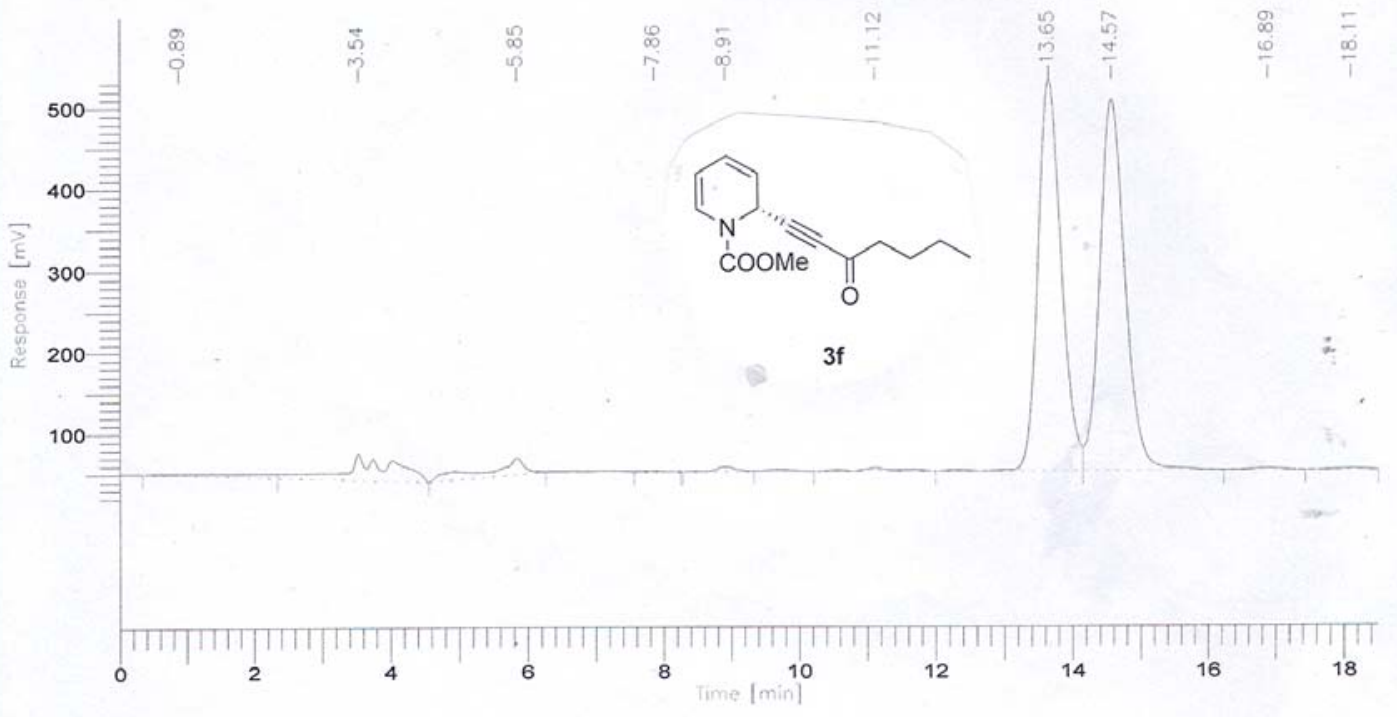

DEFAULT REPORT

\begin{tabular}{|c|c|c|c|c|c|}
\hline $\begin{array}{l}\text { Peak } \\
\#\end{array}$ & $\begin{array}{l}\text { Time } \\
\text { [min] }\end{array}$ & $\begin{array}{c}\text { Area } \\
{\left[u v^{*} s e c\right]}\end{array}$ & $\begin{array}{l}\text { Height } \\
\text { [uv] }\end{array}$ & $\begin{array}{l}\text { Area } \\
{\left[\frac{8}{8}\right]}\end{array}$ & BL \\
\hline 1 & 0.892 & 332721.80 & 1621.89 & 1.26 & BV \\
\hline 2 & 3.539 & 1659390.20 & 33403.05 & 6.28 & VB \\
\hline 3 & 5.849 & 787026.00 & 19816.76 & 2.98 & $\mathrm{BB}$ \\
\hline 4 & 7.863 & 8275.50 & 541.90 & 0.03 & BB \\
\hline 5 & 8.912 & 103940.50 & 5610.29 & 0.39 & $\mathrm{BB}$ \\
\hline 6 & 11.115 & 136045.00 & 3864.59 & 0.51 & $\mathrm{BB}$ \\
\hline 7 & 13.650 & 11474871.49 & 480591.68 & 43.40 & $\mathrm{BV}$ \\
\hline 8 & 14.572 & 11828902.90 & 456371.42 & 44.74 & VV \\
\hline 9 & 16.886 & 70665.77 & 2165.50 & 0.27 & VV \\
\hline 10 & 18.108 & 38374.84 & 1378.15 & 0.15 & VB \\
\hline
\end{tabular}


Software Version: 4.1<2F12>

Date: $06-11-7$ 15:51

Sample Name : 5-61-2-2

Data File : D: \CS\D-2 703. RAW Date: 06-11-7 15:22

Sequence File: D: \CS \D-2.SEQ Cycle: 1 Channel : A

Instrument : 970A - 0 Rack/Vial: 0/0 Operator: DIMG-2

Sample Amount : $\overline{1} . \overline{0} 000 \quad$ Dilution Factor : 1.00

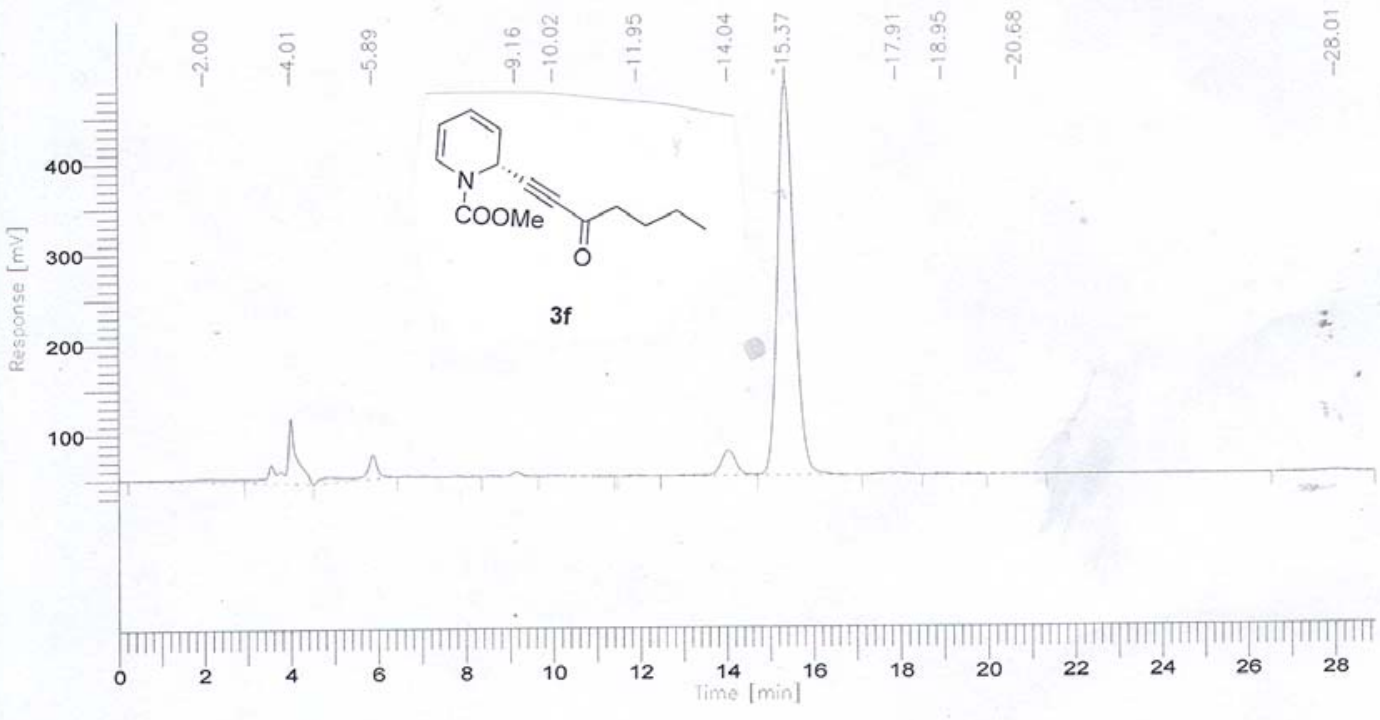

DEFAULT REPORT

\begin{tabular}{|c|c|c|c|c|c|c|}
\hline $\begin{array}{l}\text { Peak } \\
\#\end{array}$ & $\begin{array}{l}\text { Time } \\
\text { [min] }\end{array}$ & $\begin{array}{c}\text { Area } \\
{\left[u v^{\star} \text { sec }\right]}\end{array}$ & $\begin{array}{l}\text { Height } \\
\text { [uv] }\end{array}$ & $\begin{array}{l}\text { Area } \\
{[\%]}\end{array}$ & BL & \\
\hline 1 & 1.996 & 330004.47 & 2951.20 & 2.19 & BV & \\
\hline 2 & 4.011 & 1435744.53 & 72780.33 & 9.54 & VB & \\
\hline 3 & 5.891 & 654997.50 & 26761.30 & 4.35 & BB & \\
\hline 4 & 9.156 & 91070.77 & 4737.82 & 0.61 & BV & \\
\hline 5 & 10.019 & 26233.23 & 841.14 & 0.17 & VB & $91 \%$ \\
\hline 6 & 11.946 & 17335.00 & 753.89 & 0.12 & BB & \\
\hline 7 & 14.044 & 710277.37 & 28284.50 & 4.72 & BV & \\
\hline 8 & 15.373 & 11557963.39 & 436368.72 & 76.83 & VV & \\
\hline 9 & 17.908 & 98742.36 & 1993.60 & 0.66 & VV & \\
\hline 10 & 18.954 & 32974.38 & 861.74 & 0.22 & VB & \\
\hline 11 & 20.675 & 7892.00 & 205.36 & 0.05 & BB & \\
\hline 12 & 28.008 & 80319.00 & 1629.33 & 0.53 & $\mathrm{BB}$ & \\
\hline
\end{tabular}

$15043554.00 \quad 578168.93100 .00$ 


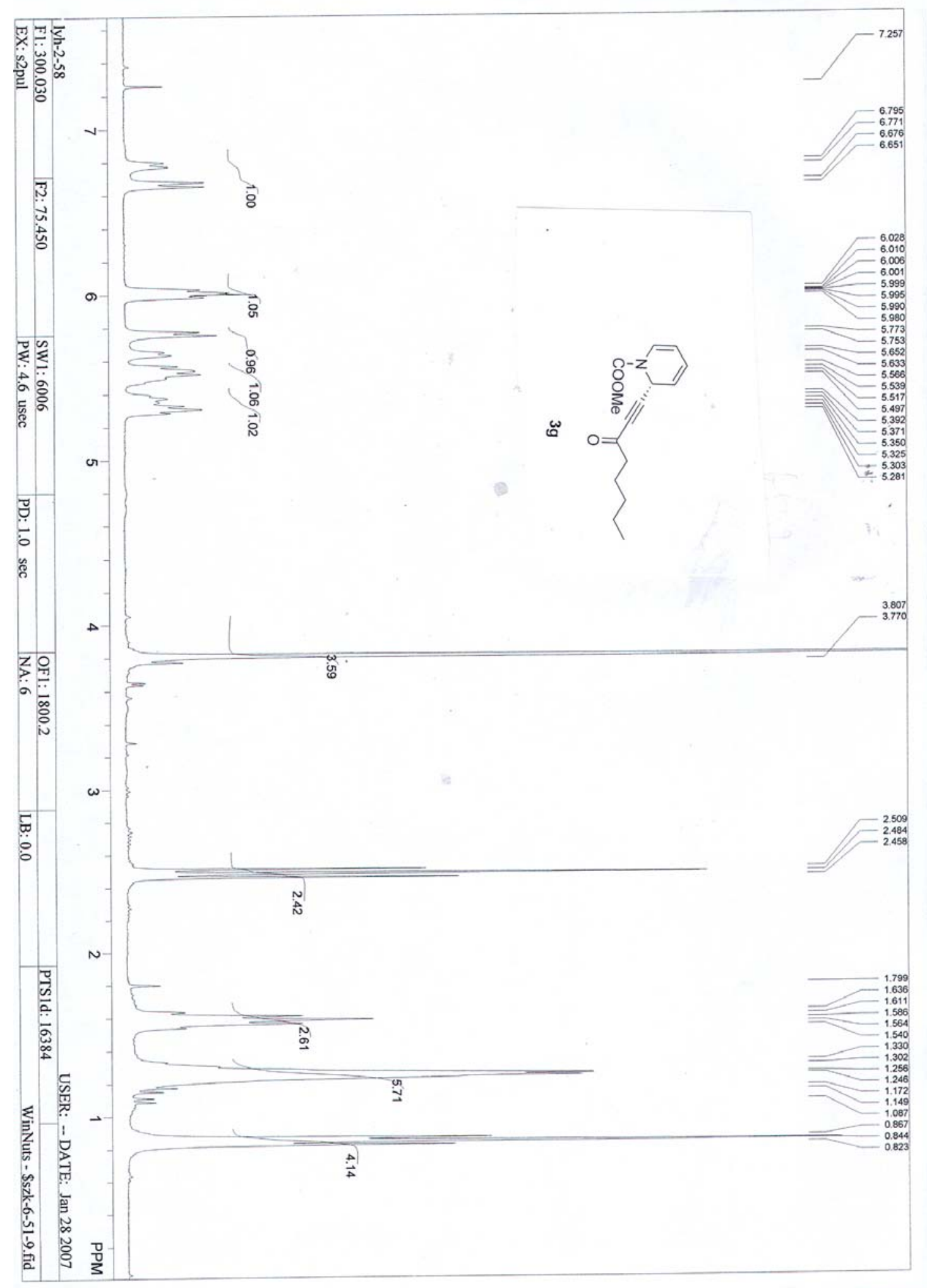




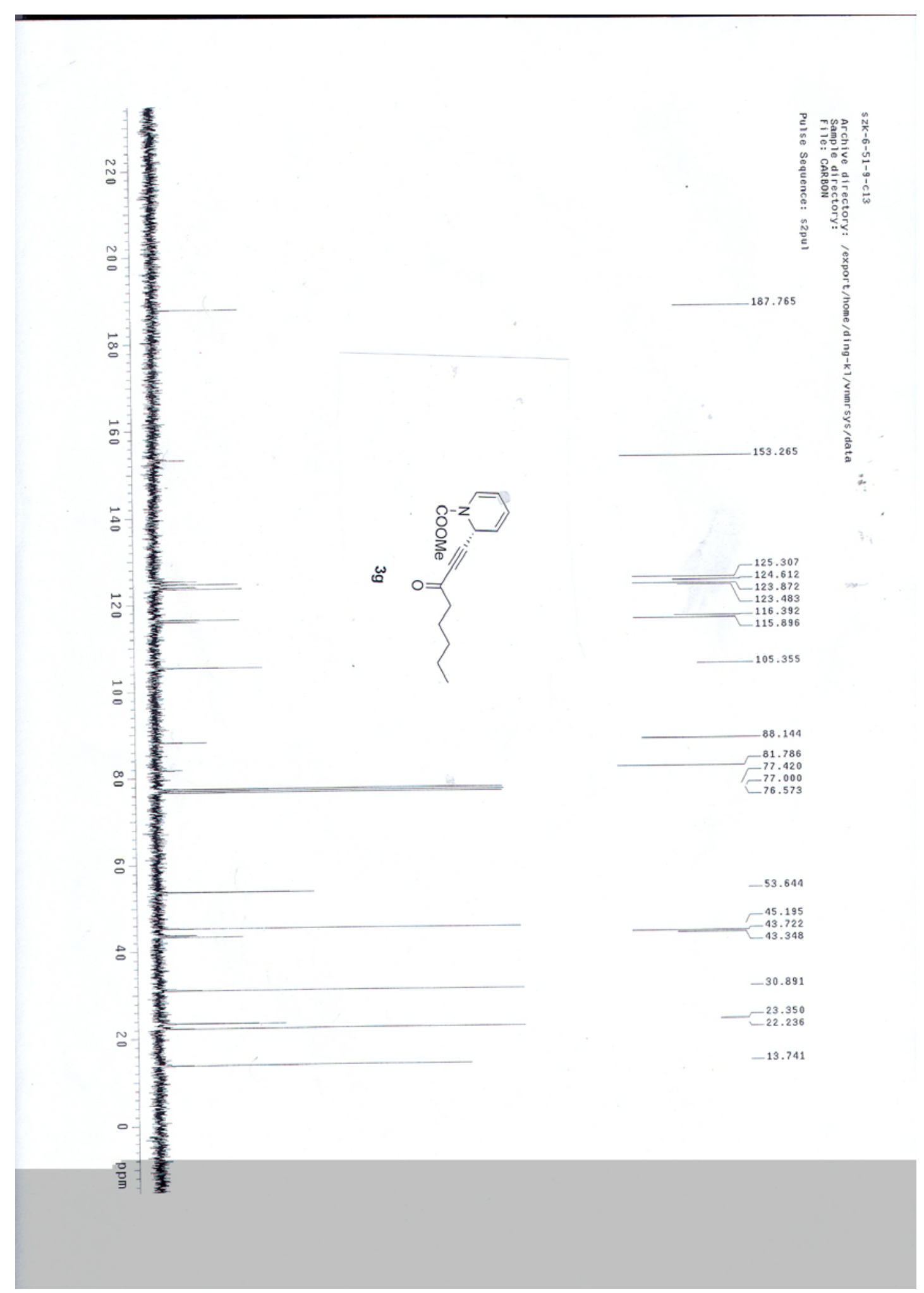




\section{色谱分析报告}

样品名称:

样品批号:

分析日期: 2007-01-16

样品文件名:6-41-2-1+-od-h. che

分析者:

分析时间: 09:47

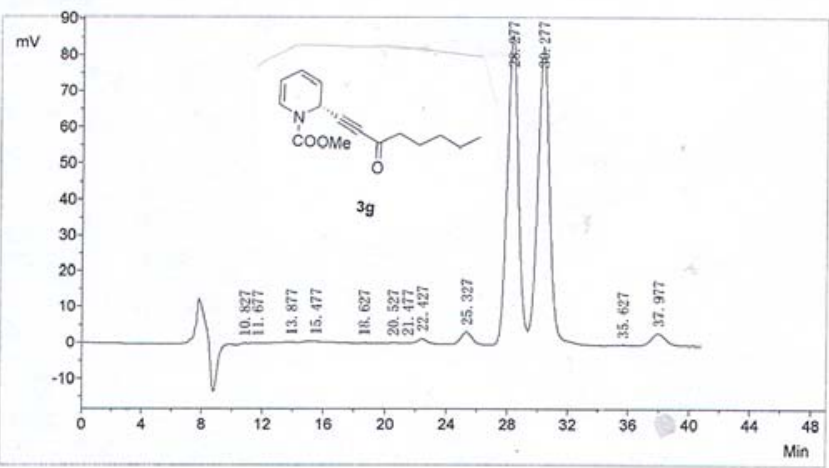

\begin{tabular}{|c|c|c|c|c|c|c|}
\hline 序号 & 峰号 & 组份名 & 保留时间 & 峰高 & 峰面积 & 面积百分比 (\%) \\
\hline 1 & 1 & & 10.827 & 430.9 & 11858.3 & 0.1235 \\
\hline 2 & 2 & & 11. 677 & 231.4 & 13633.4 & 0.1420 \\
\hline 3 & 3 & & 13.877 & 312.6 & 22590.0 & 0.2353 \\
\hline 4 & 4 & & 15.477 & 421.3 & 48813.1 & 0.5084 \\
\hline 5 & 5 & & 18.627 & 62.9 & 1771.7 & 0.0185 \\
\hline 6 & 6 & & 20.527 & 141.5 & 3587.3 & 0.0374 \\
\hline 7 & 7 & & 21.477 & 111.4 & 2585.8 & 0.0269 \\
\hline 8 & 8 & & 22.427 & 1405.0 & 59676.0 & 0.6215 \\
\hline 9 & 9 & & 25.327 & 3615.6 & 184601.0 & 1. 9225 \\
\hline 10 & 10 & & 28. 277 & 86200.1 & 4400516.3 & 45.8289 \\
\hline 11 & 11 & & 30.277 & 82759.0 & 4612677.8 & 48.0385 \\
\hline 12 & 12 & & 35.627 & 131.7 & 6855.8 & 0.0714 \\
\hline 13 & 13 & & 37.977 & 3524.0 & 232879.2 & 2.4253 \\
\hline 合计： & & & & 179347.4 & 9602045.6 & 100.0000 \\
\hline
\end{tabular}


c

\section{色谱分析报告}

样品名称：

样品批号:

分析日期:2007-01-16
样品文件名:6-41-1-2-2. che

分析者:

分析时间: 11:06

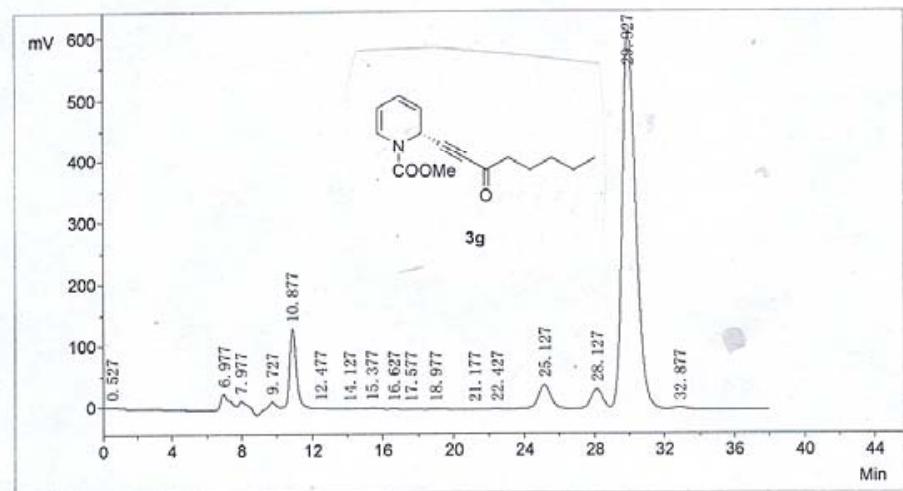

\begin{tabular}{|c|c|c|c|c|c|c|}
\hline 序号 & 峰号 & 组份名 & 保留时间 & 峰高 & 峰面积 & 面积百分比 (\%) \\
\hline 1 & 1 & & 0.527 & 969.8 & 33691.0 & 0.0668 \\
\hline 2 & 2 & & 6. 977 & 25749.9 & 975095.4 & 1. 9340 \\
\hline 3 & 3 & & 7. 977 & 16300.5 & 662078.9 & 1. 3131 \\
\hline 4 & 4 & & 9.727 & 21918.9 & 1010340.5 & 2.0039 \\
\hline 5 & 5 & & 10.877 & 138095.5 & 10496972.9 & 20.8194 \\
\hline 6 & 6 . & & 12. 477 & 564.2 & 12870.9 & 0.0255 \\
\hline 7 & 7 & & 14. 127 & 609.3 & 17187.9 & 0.0341 \\
\hline 8 & 8 & & 15. 377 & 1493.2 & 96928.5 & 0.1922 \\
\hline 9 & 9 & & 16. 627 & 1122.5 & 32814.5 & 0.0651 \\
\hline 10 & 10 & & 17. 577 & 605.1 & 22315.4 & 0.0443 \\
\hline 11 & 11 & & 18. 977 & 873.4 & 43507.0 & 0.0863 \\
\hline 12 & 12 & & 21.177 & 332.4 & 6292.9 & 0.0125 \\
\hline 13 & 13 & & 22.427 & 1304.8 & 47495.3 & 0.0942 \\
\hline 14 & 14 & & 25.127 & 39990.4 & 2100341.2 & 4. 1658 \\
\hline 15 & 15 & & 28.127 & 33149.8 & 1659595.7 & 3. 2916 \\
\hline 16 & 16 & & 29.927 & 616186.2 & 32999106.3 & 65.4494 \\
\hline 17 & 17 & & 32.877 & 3432.9 & 202630.6 & 0.4019 \\
\hline
\end{tabular}

$9 \%$ 


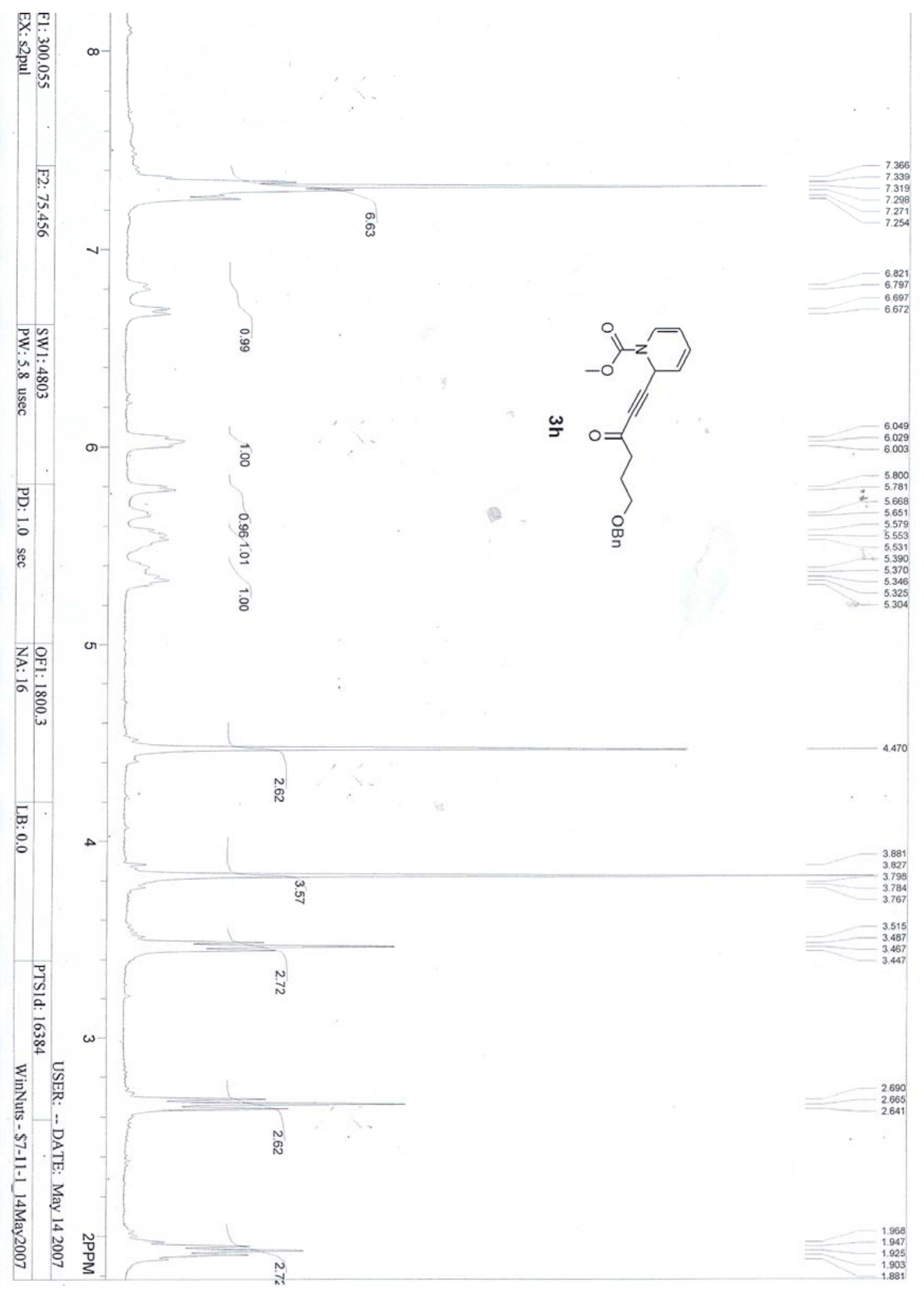




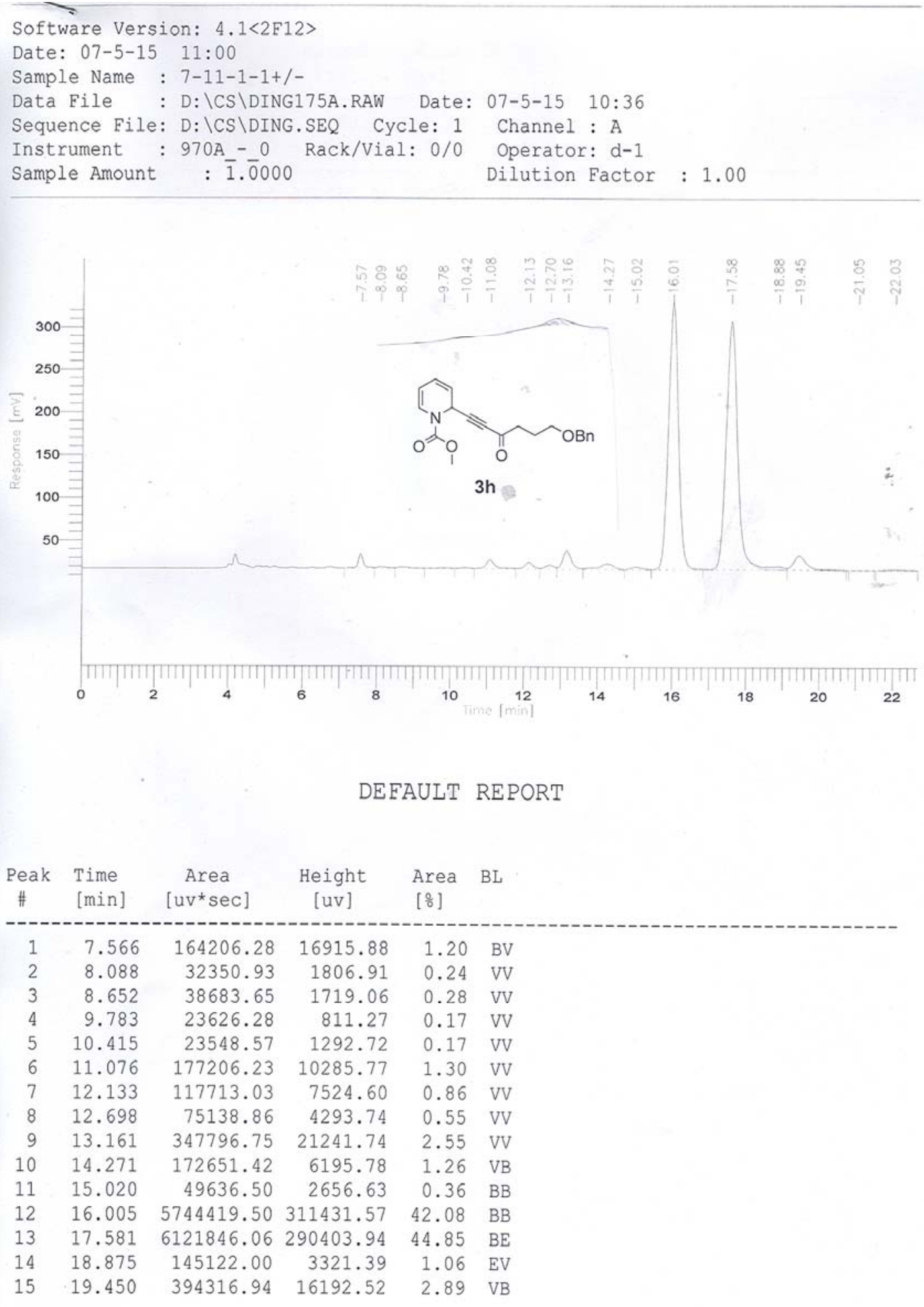


Software Version: $4.1<2 \mathrm{~F} 12\rangle$

Date: $07-5-15$ 14:57

Sample Name : 7-11-1-3

Data File : D: \CS\DING177A. RAW Date: 07-5-15 14:31

Sequence File: D: \CS\DING.SEQ Cycle: 1 Channel : A

Instrument : 970A - 0 Rack/Vial: 0/0 Operator: d-1

Sample Amount : $\overline{1} . \overline{0} 000 \quad$ Dilution Factor : 1.00

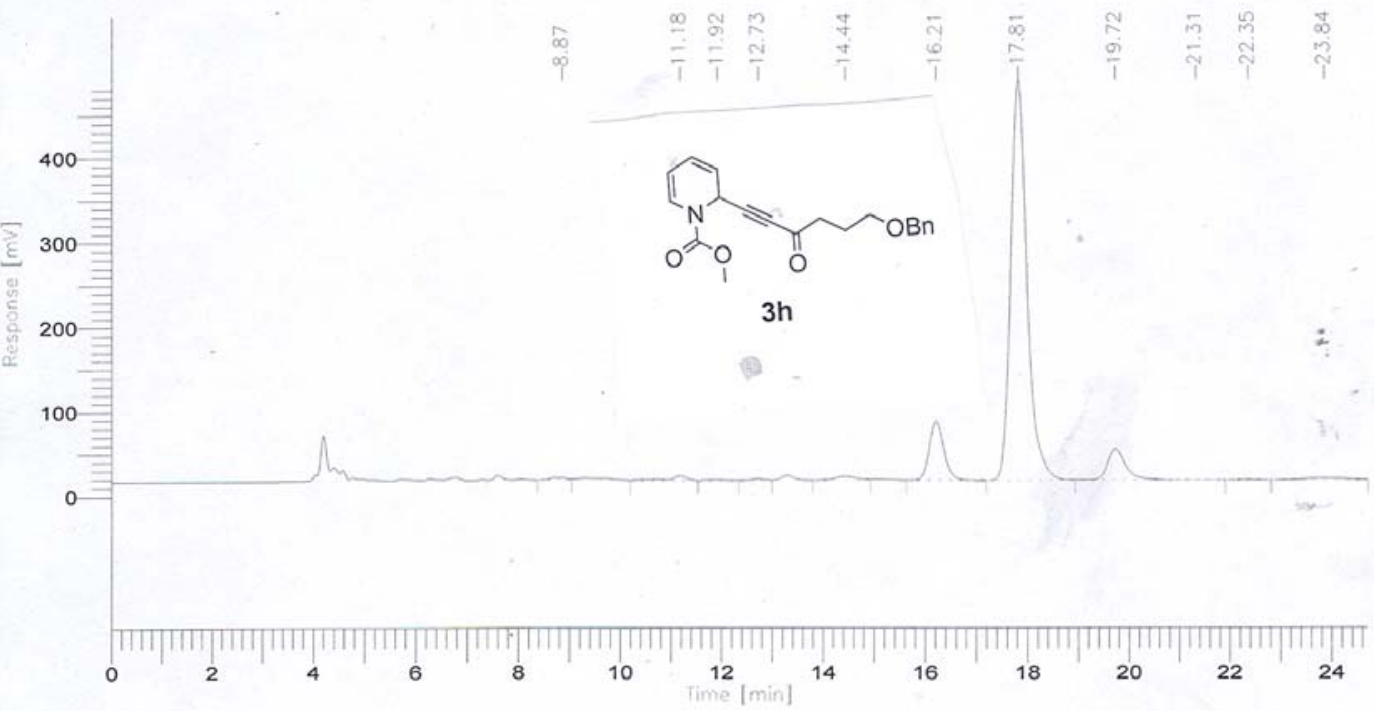

DEFAULT REPORT

\begin{tabular}{rrrrrr}
$\begin{array}{c}\text { Peak } \\
\text { \# }\end{array}$ & $\begin{array}{l}\text { Time } \\
\text { [min] }\end{array}$ & \multicolumn{1}{c}{$\begin{array}{c}\text { Area } \\
\text { [uv*sec }]\end{array}$} & \multicolumn{1}{c}{$\begin{array}{c}\text { Height } \\
\text { [uv] }\end{array}$} & $\begin{array}{l}\text { Area } \\
{[\%]}\end{array}$ & BL \\
\hline 1 & 8.865 & 41336.00 & 2014.31 & 0.31 & BB \\
2 & 11.181 & 122761.00 & 5221.06 & 0.93 & BB \\
3 & 11.919 & 15801.97 & 714.68 & 0.12 & BV \\
4 & 12.728 & 26912.03 & 1851.37 & 0.20 & VB \\
5 & 14.441 & 103309.00 & 3808.07 & 0.78 & BB \\
6 & 16.210 & 1385181.50 & 69918.34 & 10.47 & BB \\
7 & 17.811 & 10457548.06 & 472030.95 & 79.07 & BV \\
8 & 19.718 & 941154.94 & 37427.84 & 7.12 & VE \\
9 & 21.308 & 5587.00 & 172.75 & 0.04 & BB \\
10 & 22.352 & 16961.50 & 715.40 & 0.13 & BB \\
11 & 23.838 & 109516.00 & 1871.40 & 0.83 & BB
\end{tabular}

$13226069.00 \quad 595746.17 \quad 100.00$ 


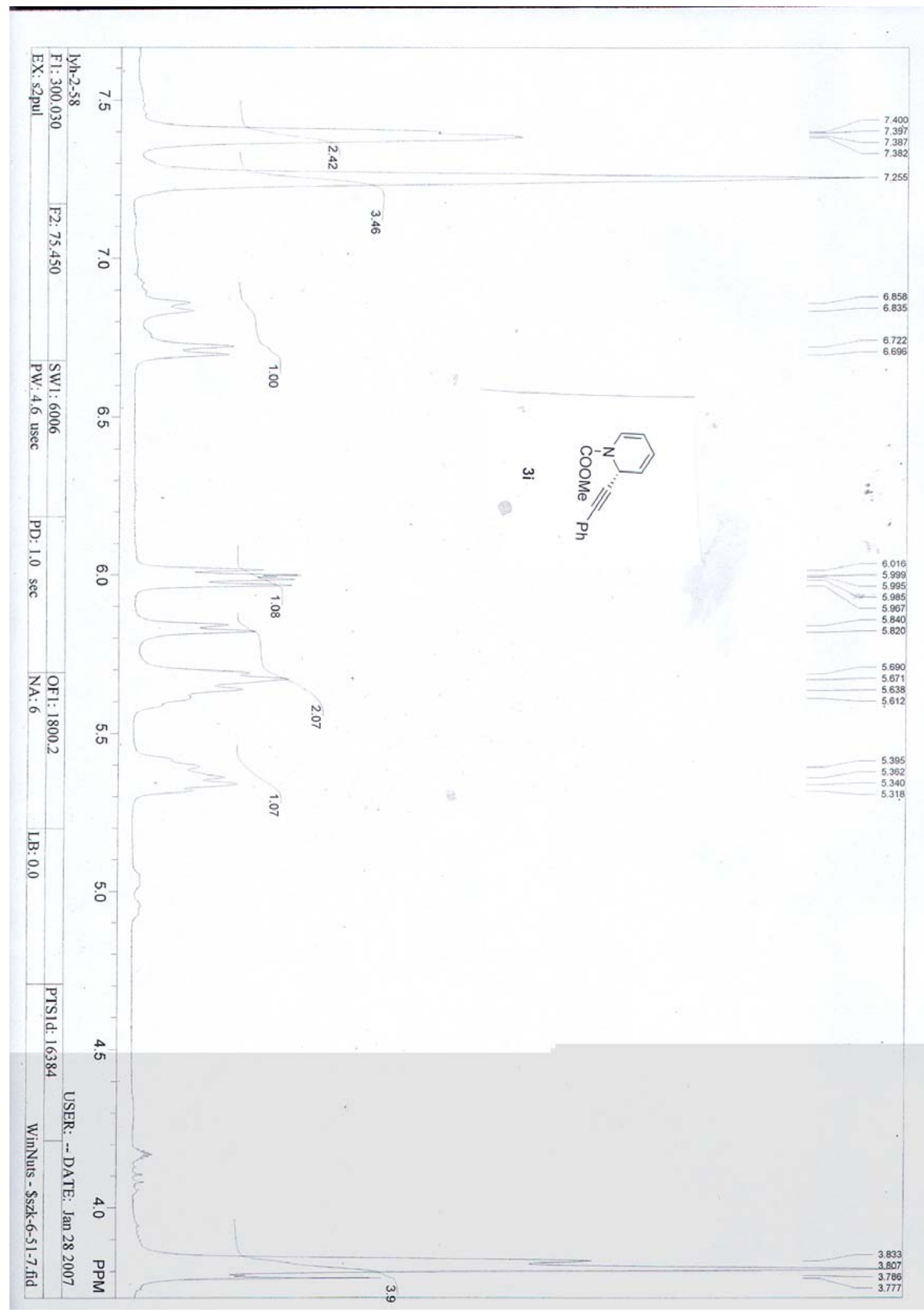




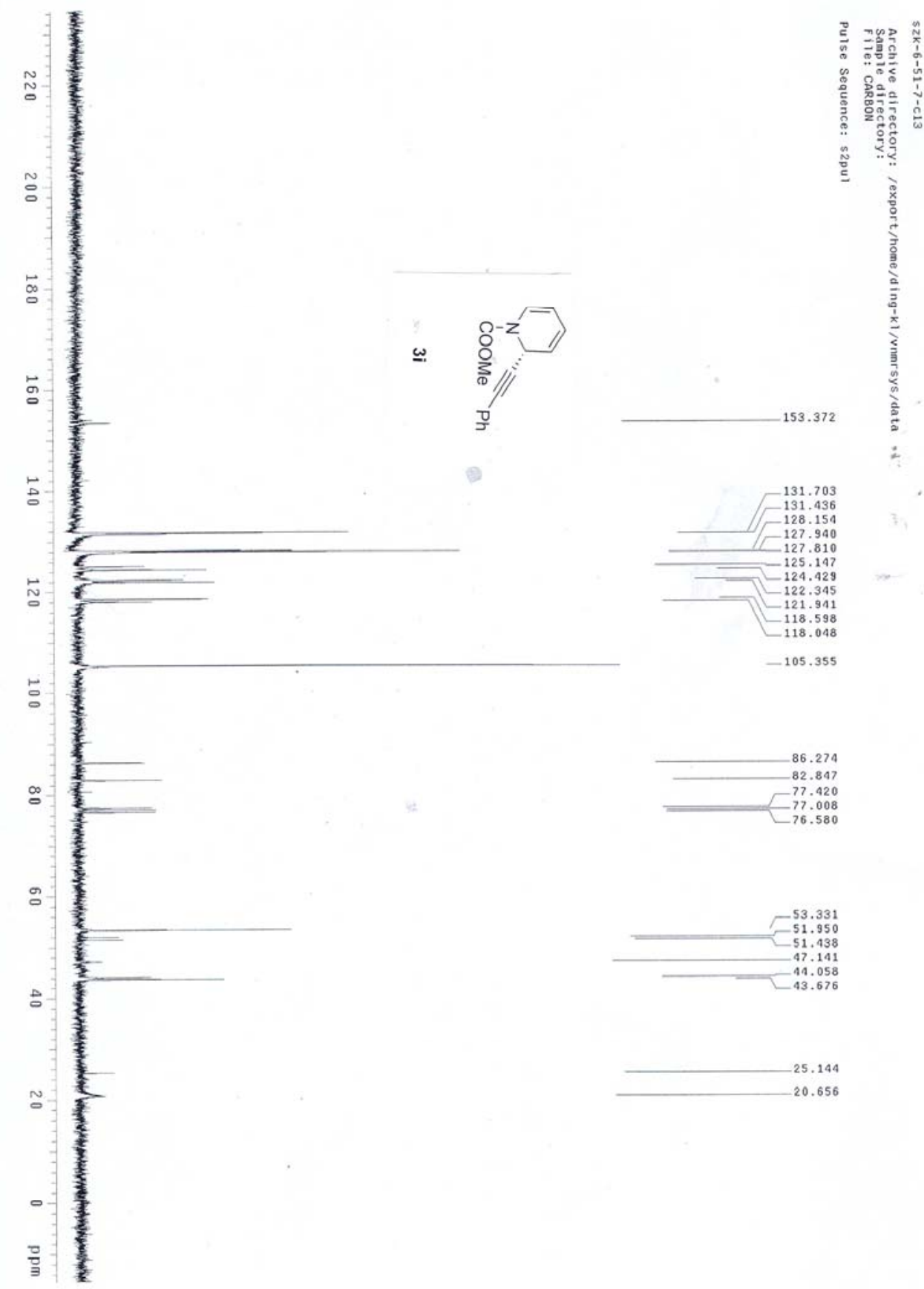




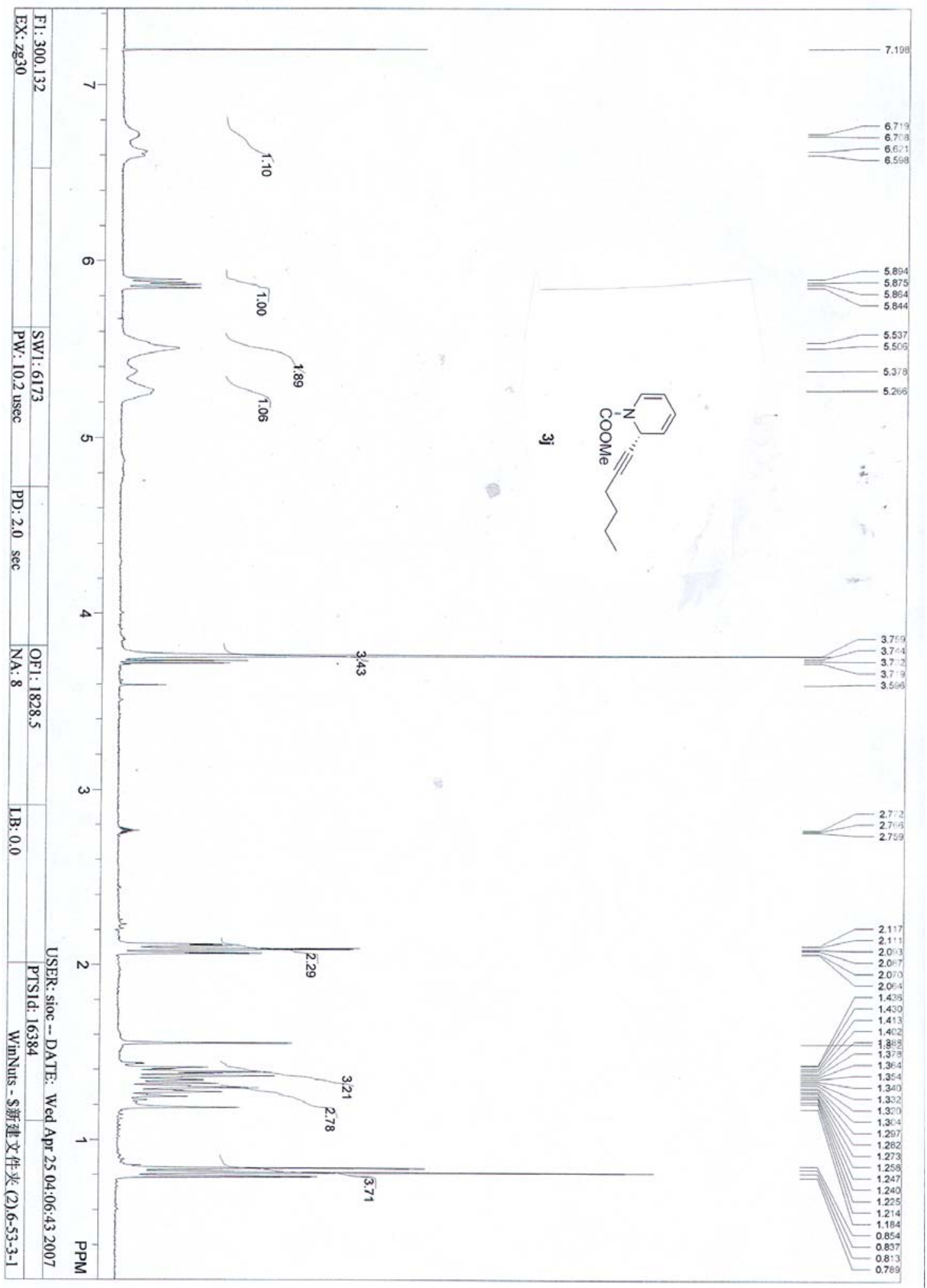




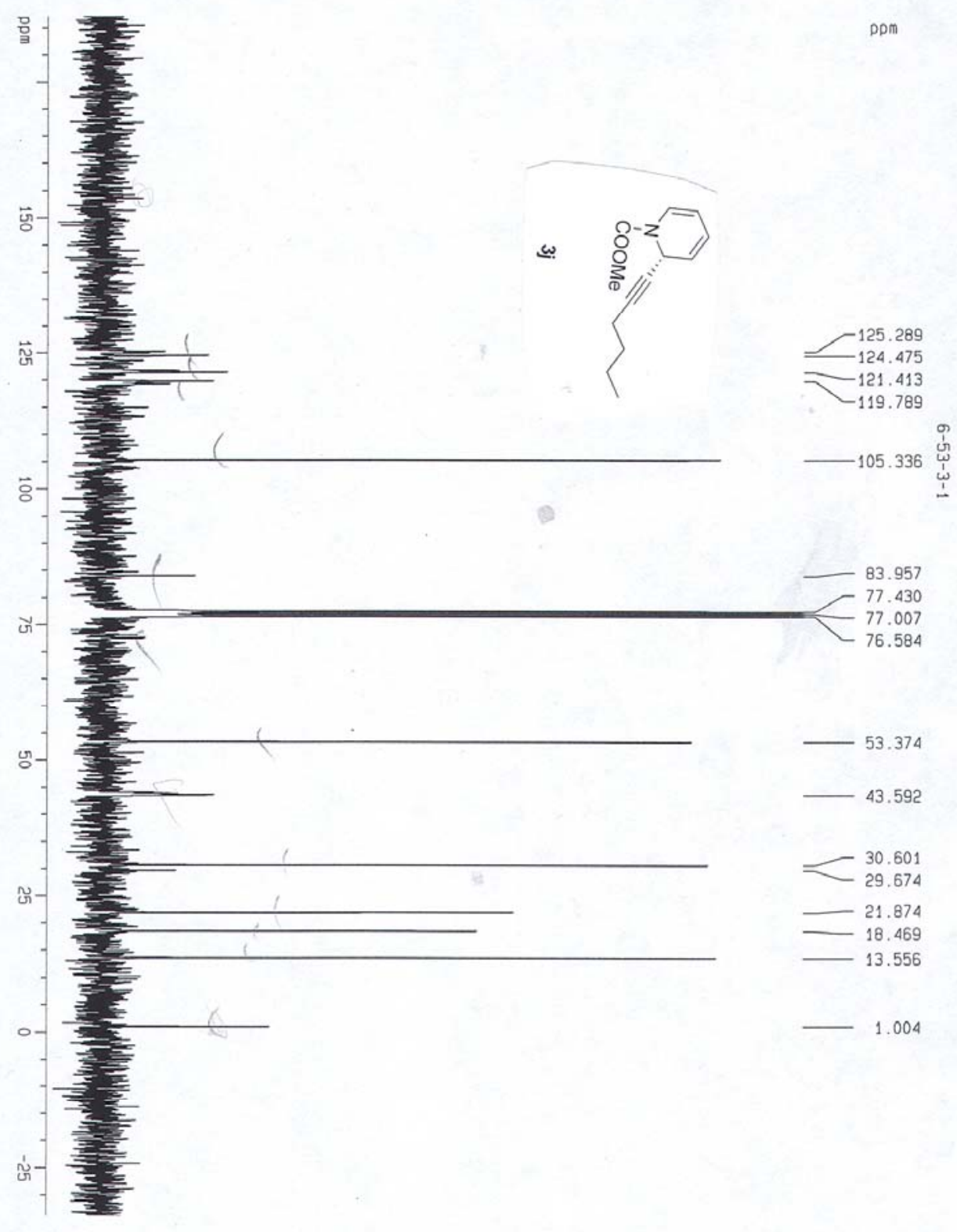




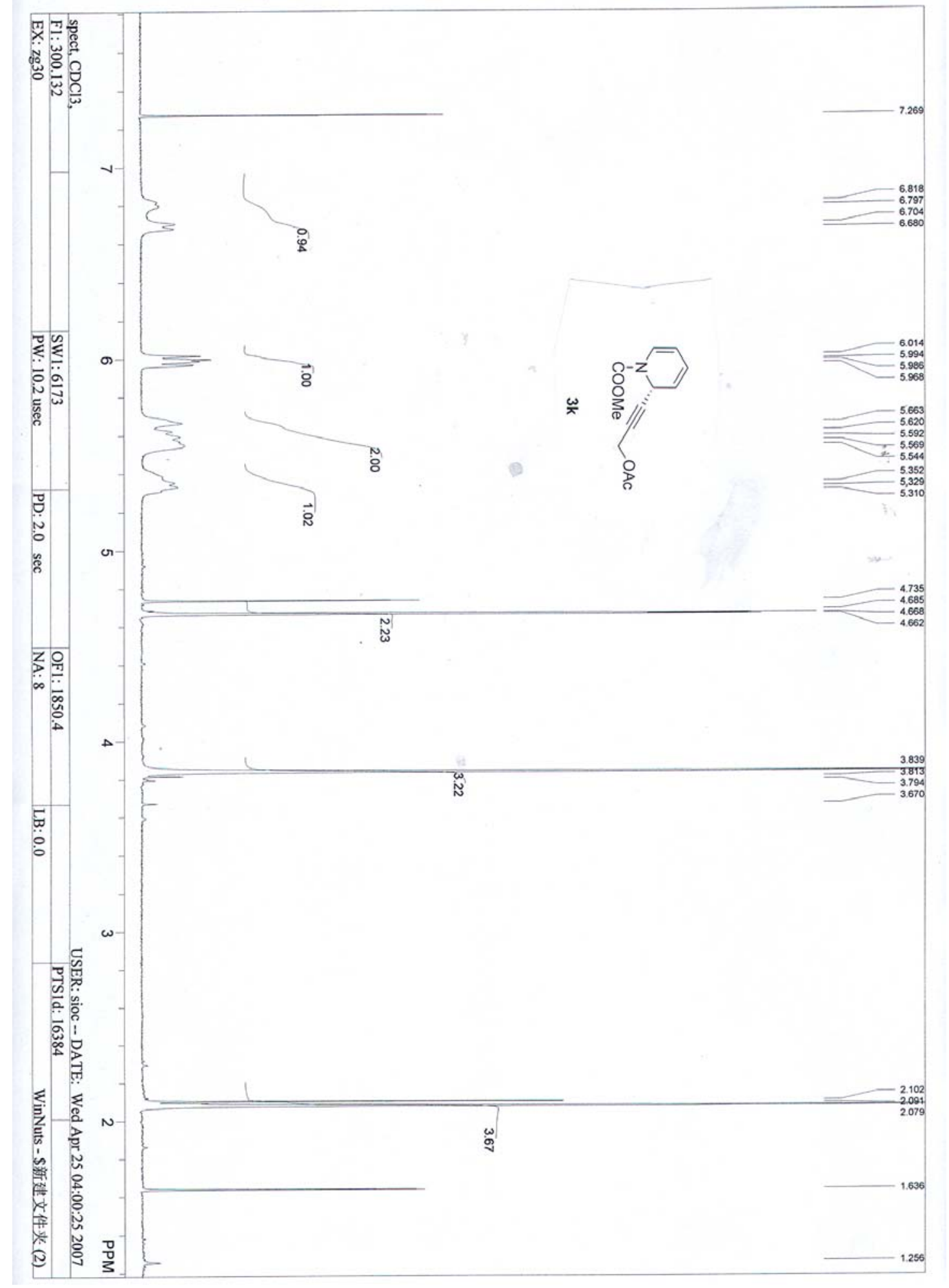




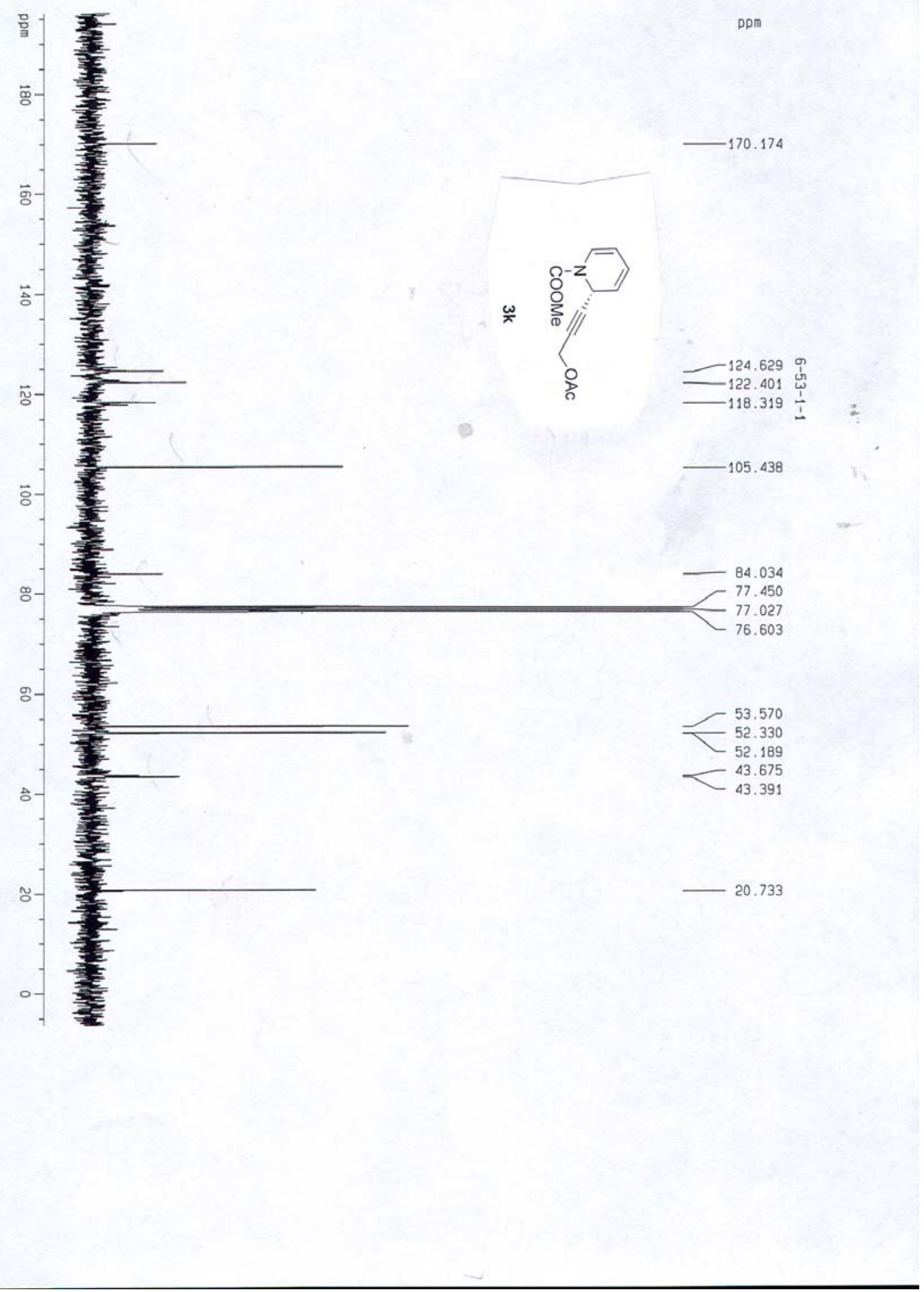




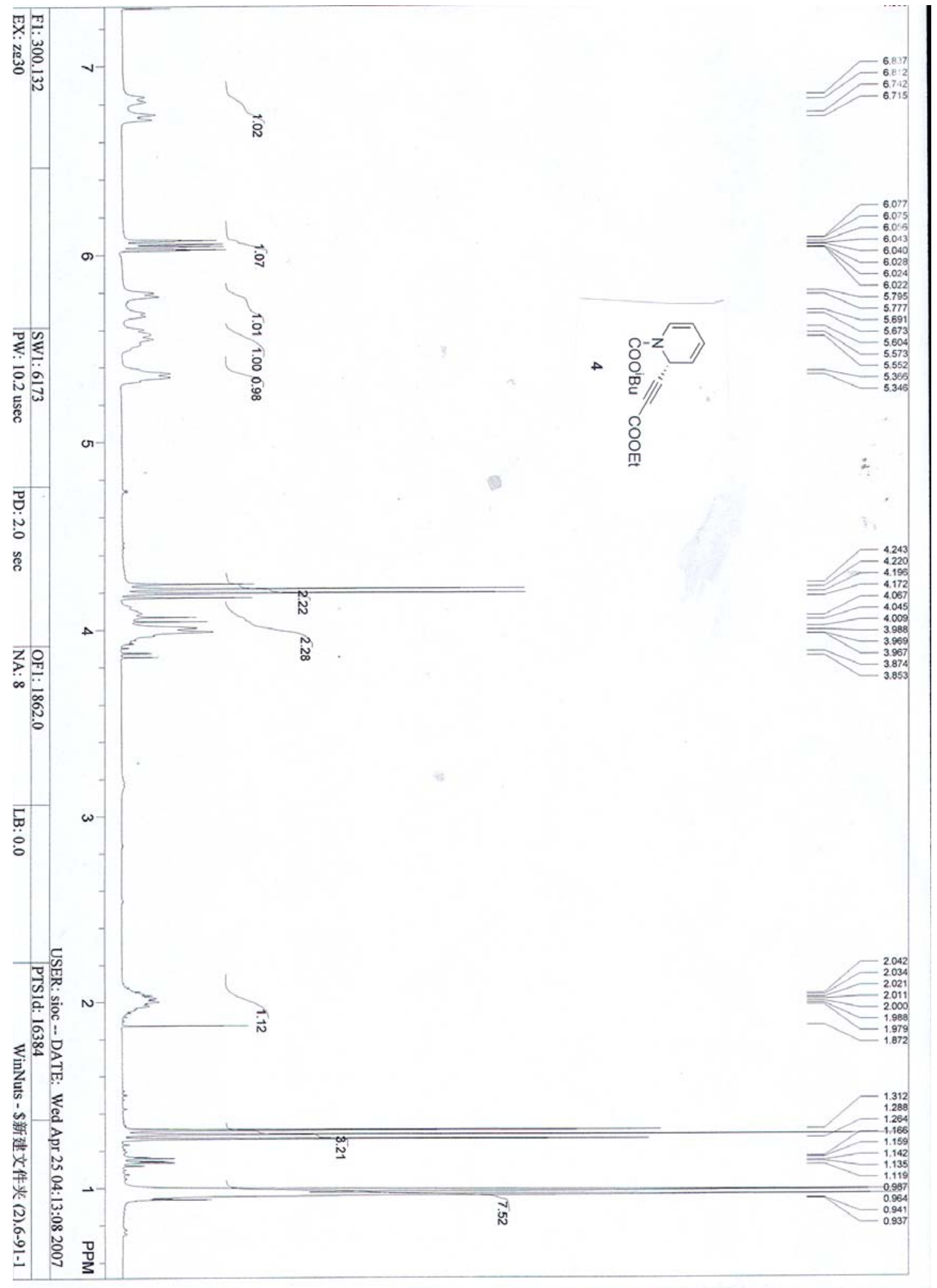




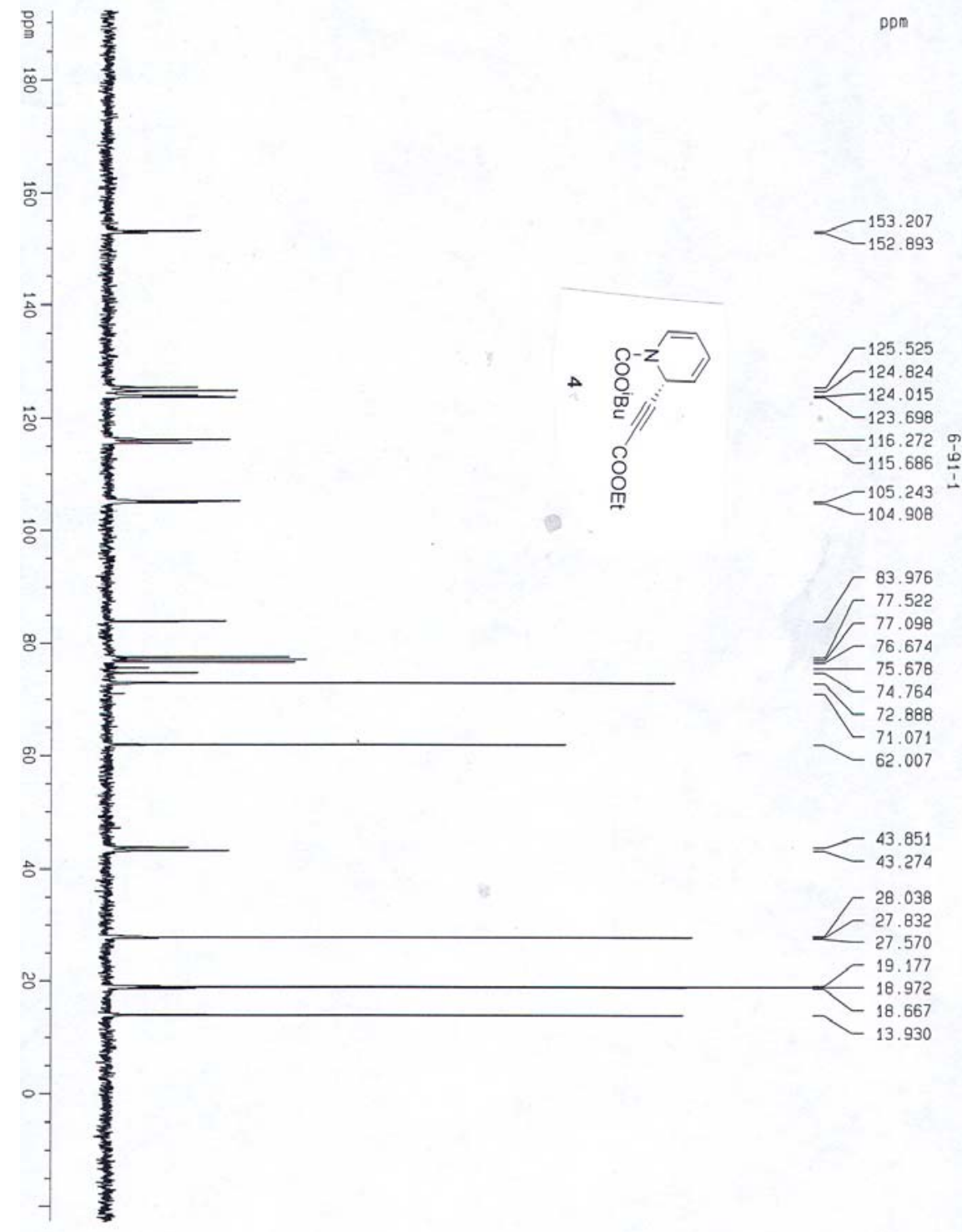


Software Version: 4.1<2F12>

Date: 06-9-12 9:10

Sample Name : 5-83-1-1+-

Data File : D: \CS\D-2_461. RAW Date: 06-9-12 8:43

Sequence File: D: \CS\D-2.SEQ Cycle: 1 Channel : A

Instrument : 970A_-_0 Rack/Vial: 0/0 Operator: DING

Sample Amount : $\overline{1} . \overline{0} 000 \quad$ Dilution Factor $: 1.00$

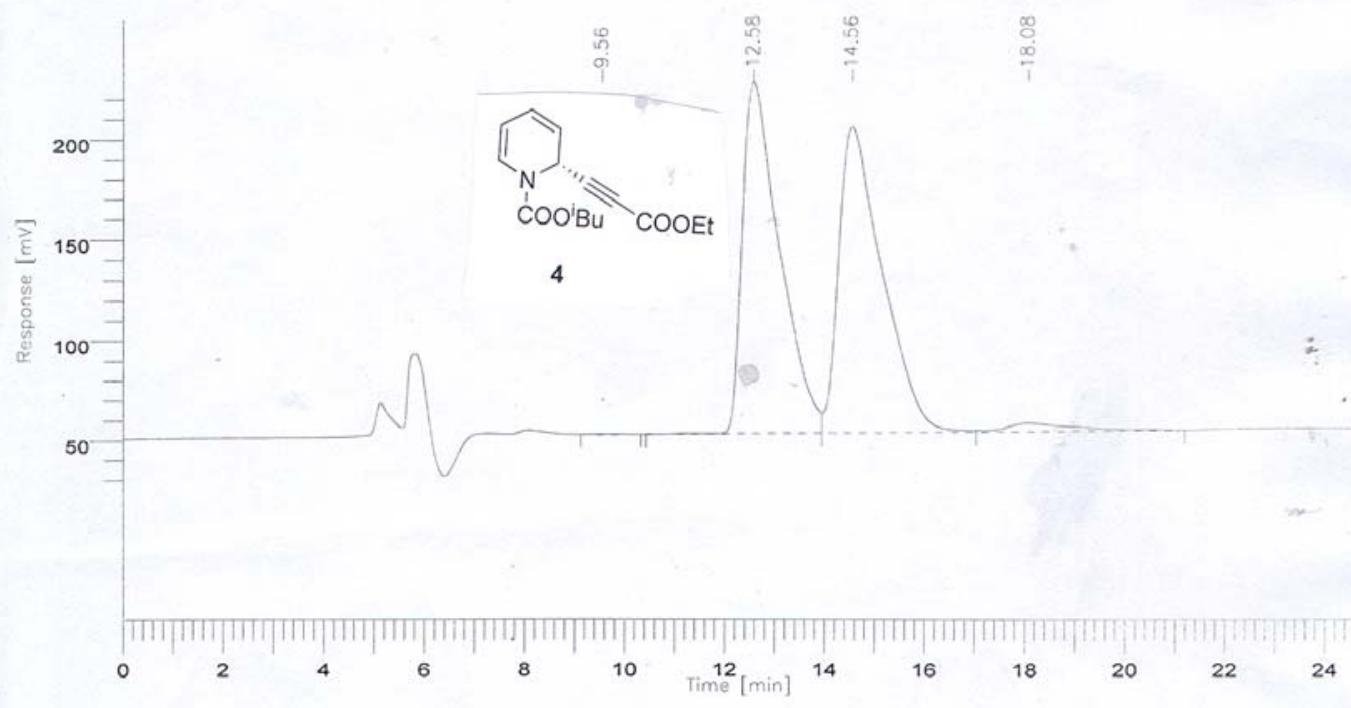

DEFAULT REPORT

\begin{tabular}{|c|c|c|c|c|c|}
\hline $\begin{array}{l}\text { Peak } \\
\#\end{array}$ & $\begin{array}{l}\text { Time } \\
\text { [min] }\end{array}$ & $\begin{array}{c}\text { Area } \\
{[\text { uv*sec] }}\end{array}$ & $\begin{array}{l}\text { Height } \\
\text { [uv] }\end{array}$ & $\begin{array}{c}\text { Area } \\
{[\%]}\end{array}$ & BL \\
\hline 1 & 9.558 & 7179.00 & 162.44 & 0.04 & BB \\
\hline 2 & 12.584 & 9067653.88 & 175064.17 & 48.36 & BV \\
\hline 3 & 14.558 & 9253710.98 & 152289.51 & 49.35 & VV \\
\hline 4 & 18.079 & 422327.64 & 4465.62 & 2.25 & VB \\
\hline
\end{tabular}


Software Version: 4.1<2F12〉

Date: 06-9-12 9:42

Sample Name : 5-83-1-2

Data File : D: \CS \D-2_462. RAW Date: 06-9-12 9:14

Sequence File: D: \CS \D-2.SEQ Cycle: 1 Channel : A

Instrument : 970A_-_0 Rack/Vial: 0/0 Operator: DING

Sample Amount : 1.0000 Dilution Factor : 1.00

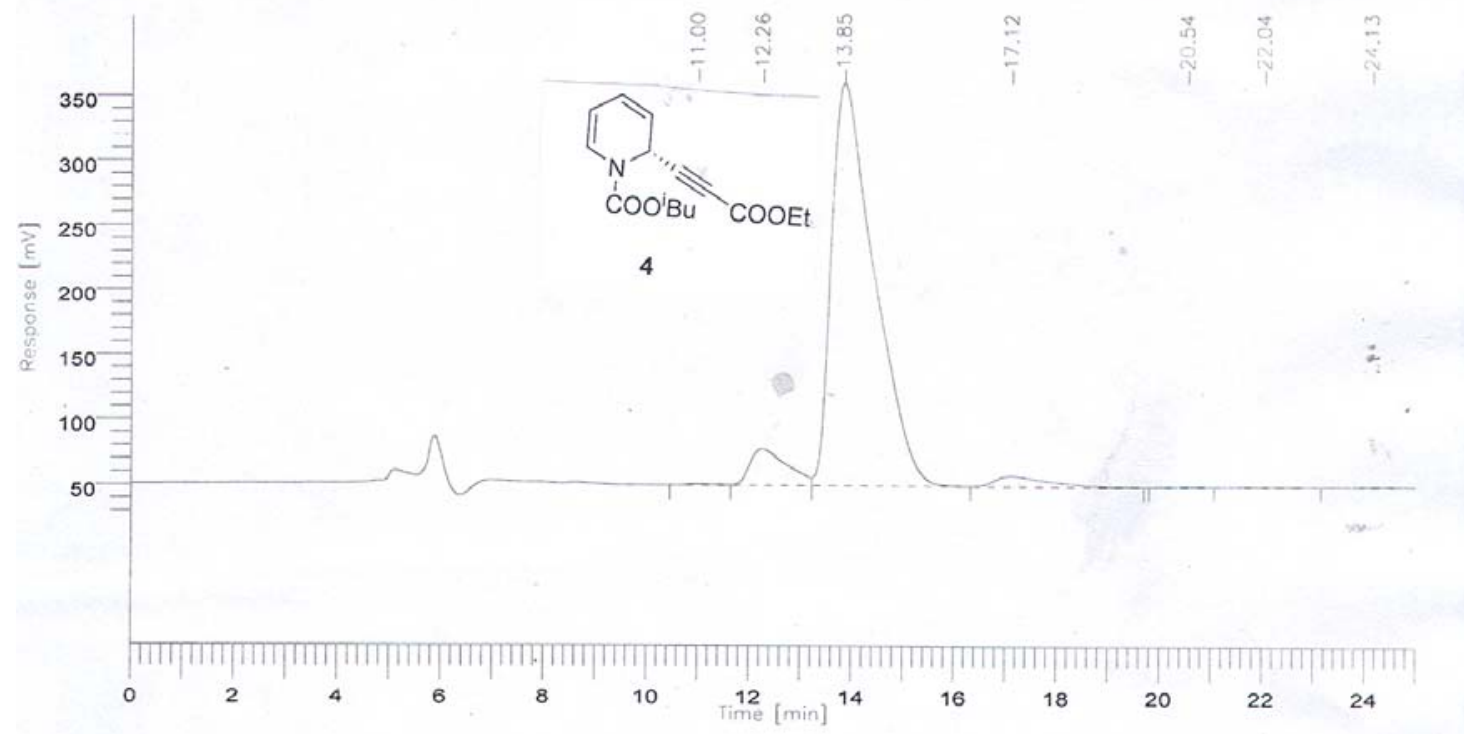

DEFAULT REPORT

\begin{tabular}{ccrrrr}
$\begin{array}{c}\text { Peak } \\
\text { \# }\end{array}$ & $\begin{array}{l}\text { Time } \\
\text { [min] }\end{array}$ & \multicolumn{1}{c}{$\begin{array}{c}\text { Area } \\
\text { [uv*sec] }\end{array}$} & \multicolumn{1}{c}{$\begin{array}{c}\text { Height } \\
\text { [uv] }\end{array}$} & $\begin{array}{l}\text { Area } \\
\text { [\%] }\end{array}$ & BL \\
\hdashline 1 & 10.997 & 28920.45 & 708.51 & 0.14 & BV \\
2 & 12.262 & 1381968.65 & 27795.24 & 6.91 & VV \\
3 & 13.848 & 17965578.20 & 309633.95 & 89.87 & VV \\
4 & 17.119 & 558145.53 & 7884.77 & 2.79 & VB \\
5 & 20.542 & 12987.18 & 287.49 & 0.06 & BV \\
6 & 22.035 & 25542.06 & 319.43 & 0.13 & VV \\
7 & 24.128 & 17279.76 & 288.05 & 0.09 & VB
\end{tabular}

$19990421.84 \quad 346917.44 \quad 100.00$

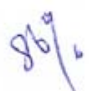




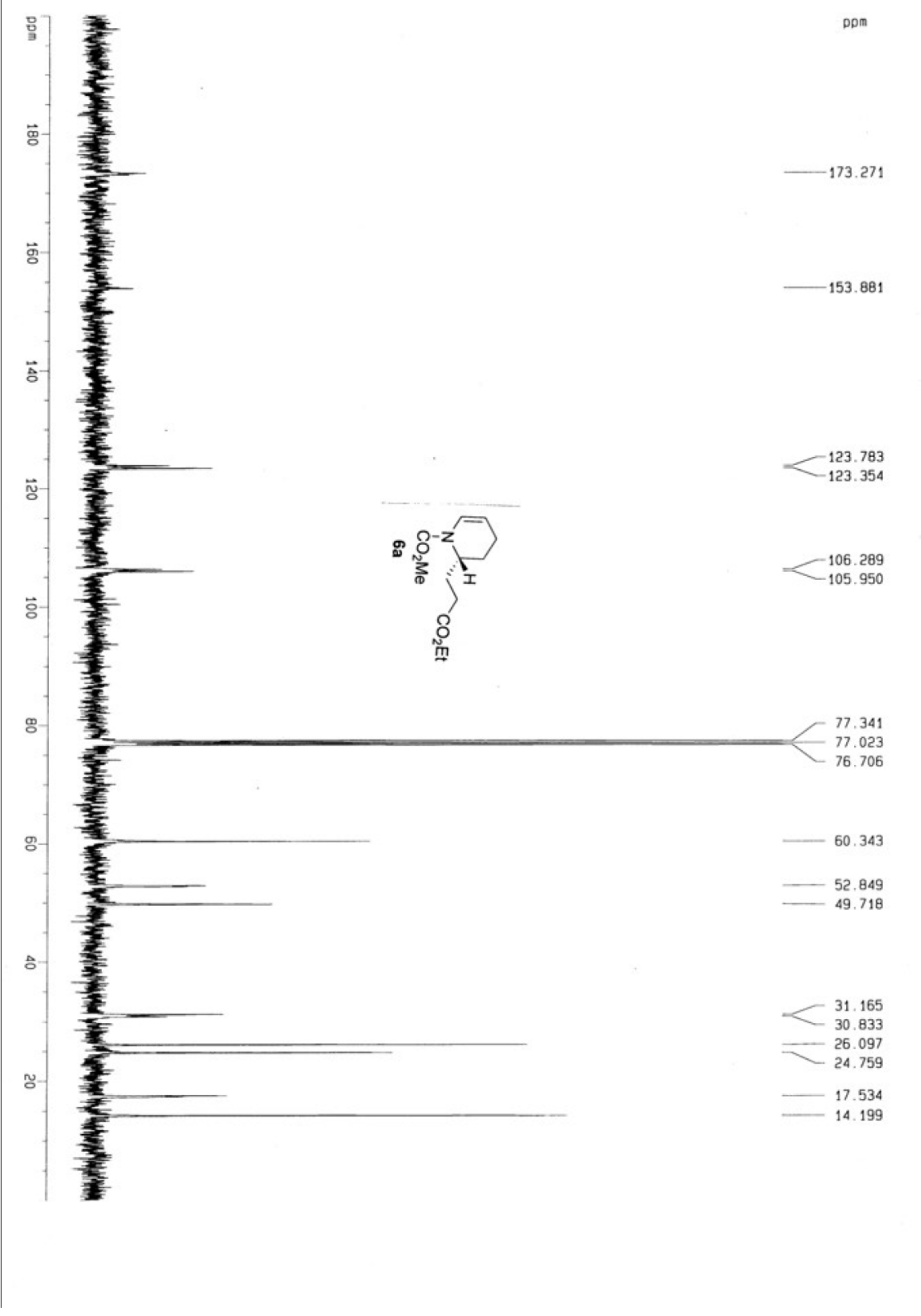




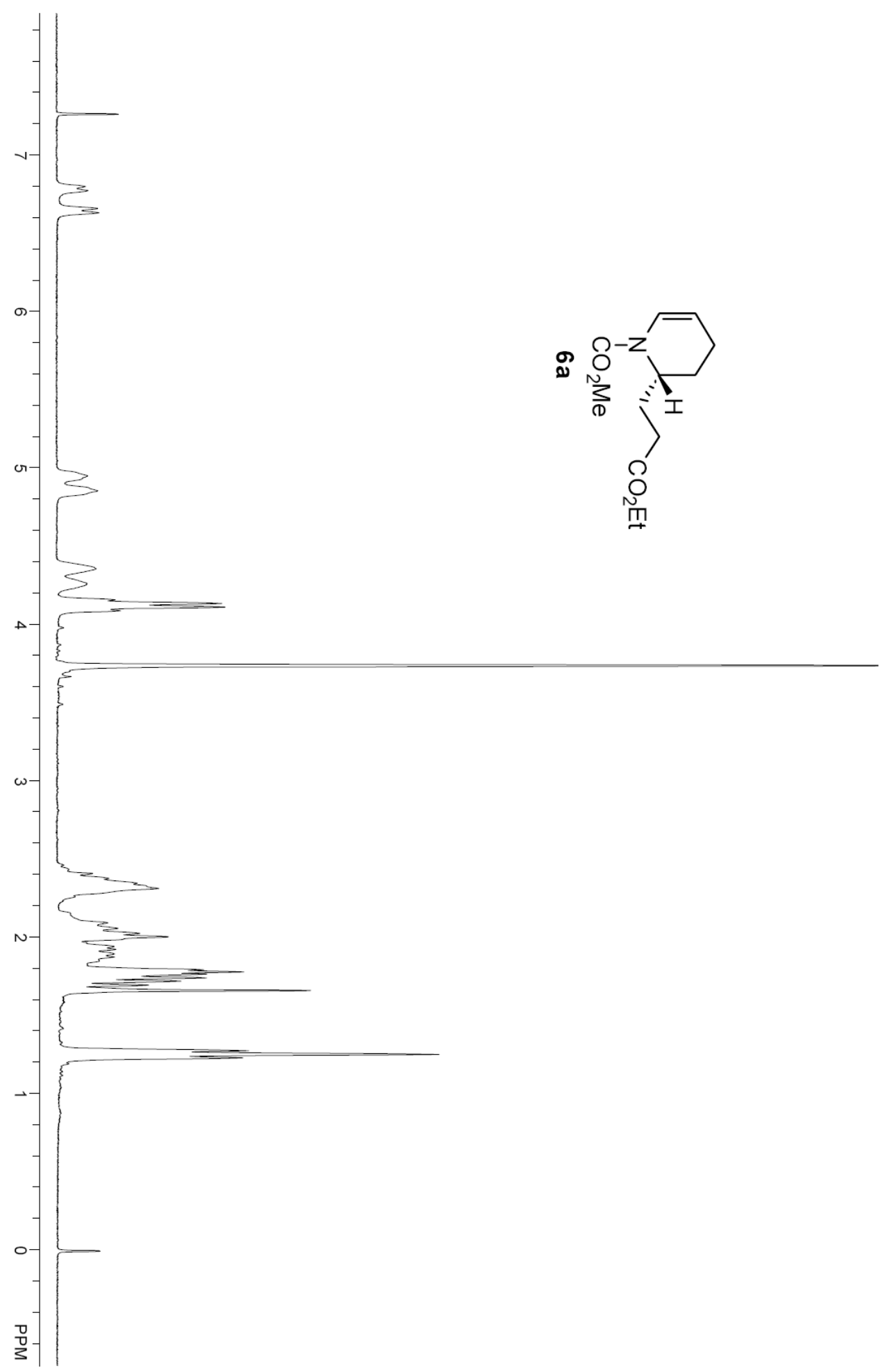



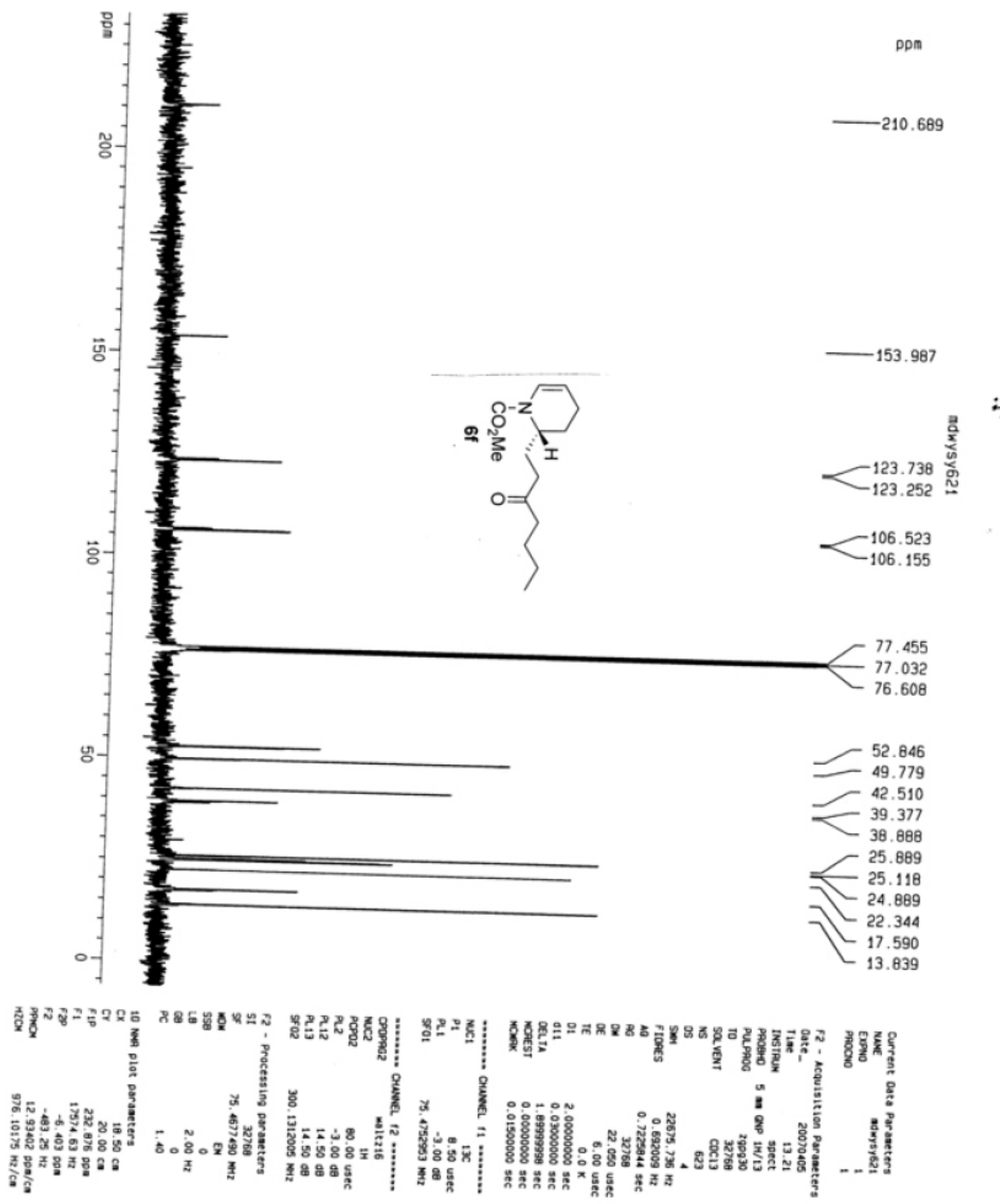


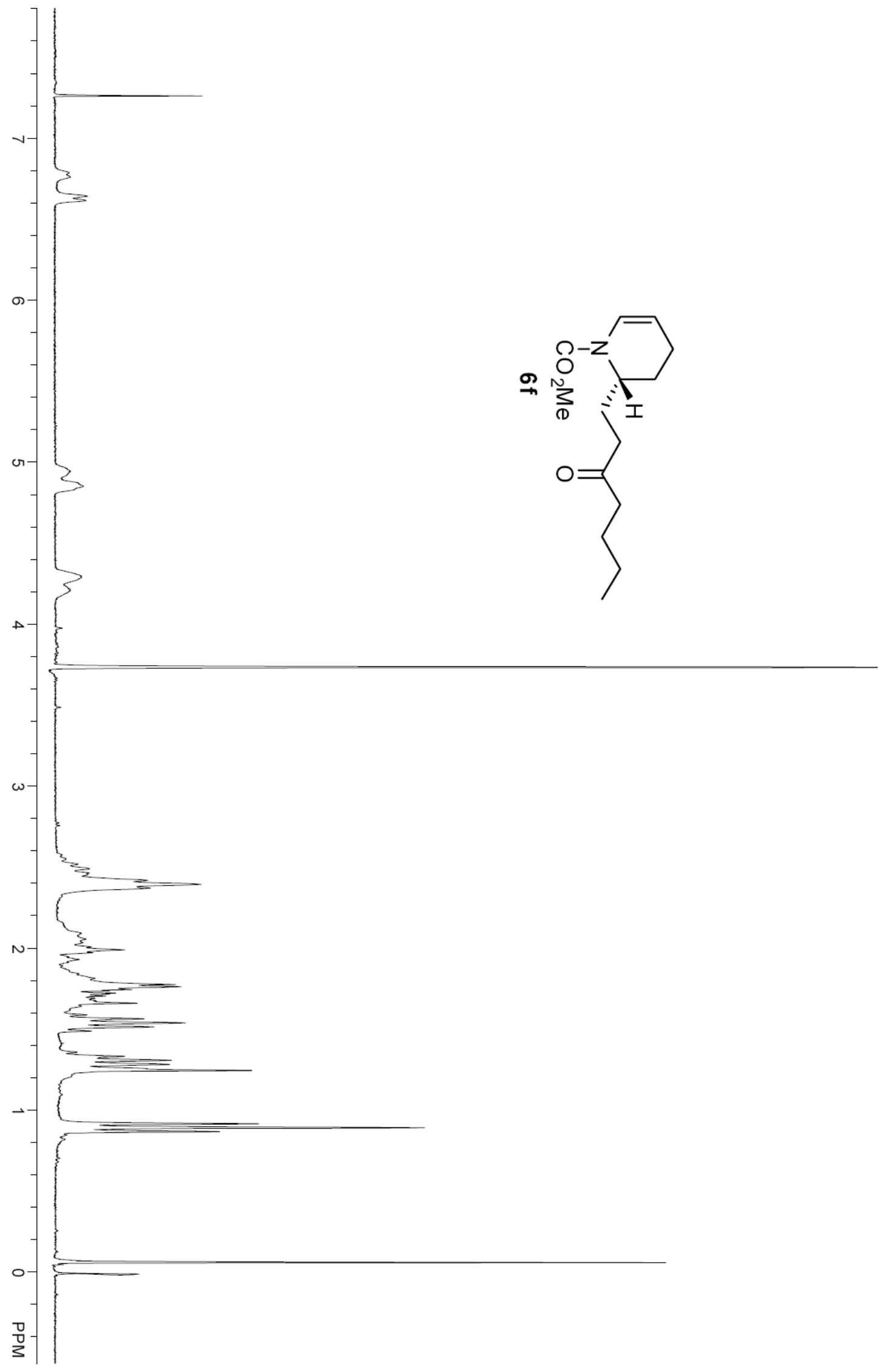




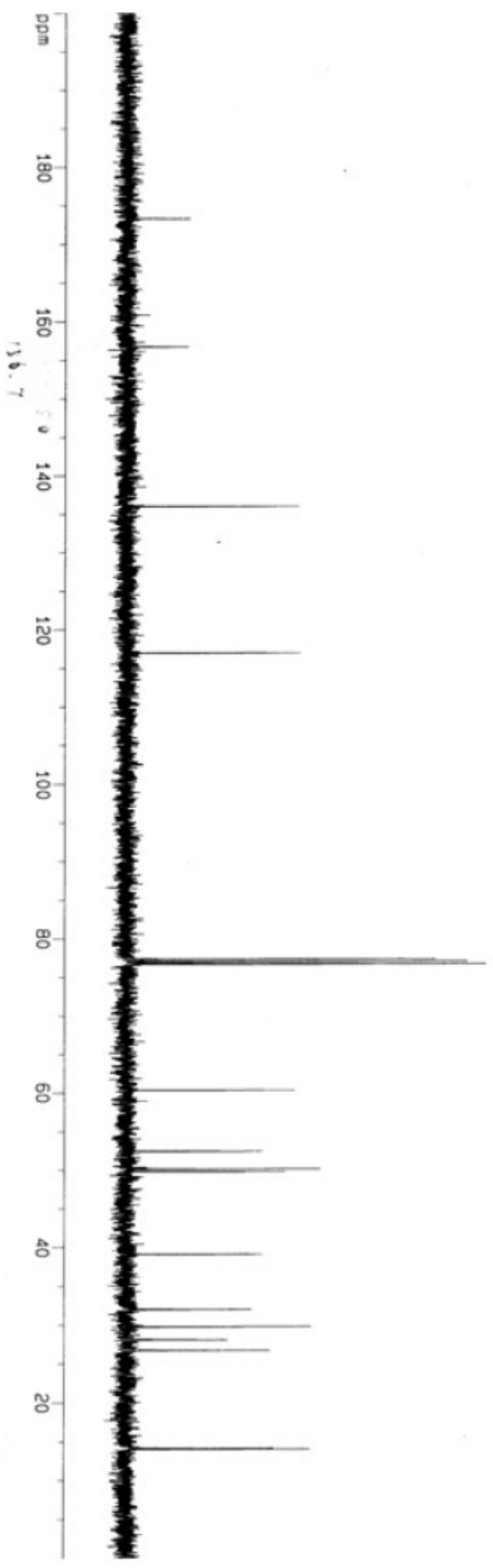

ppm
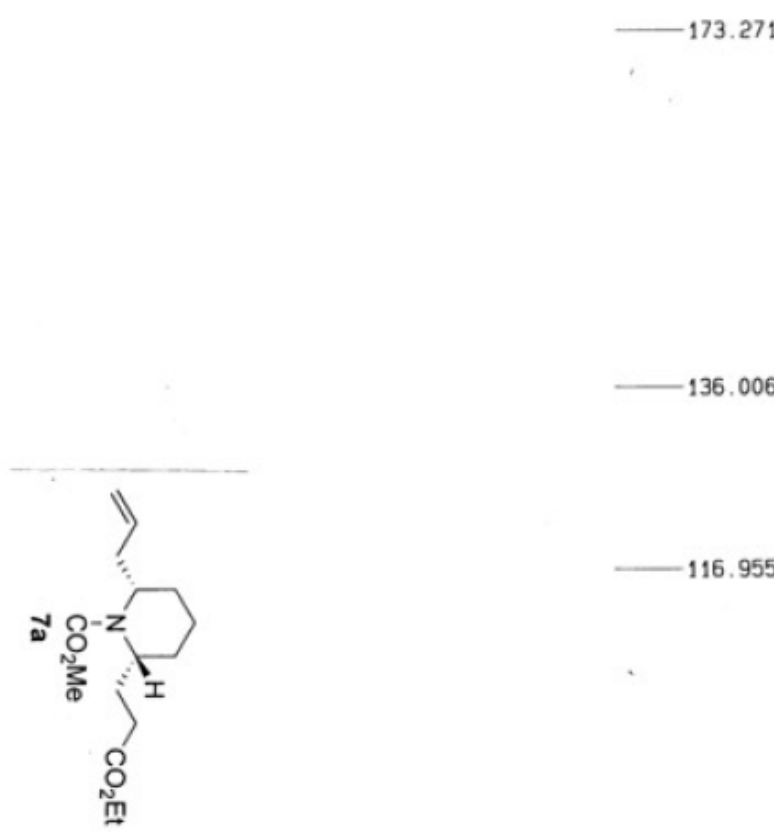


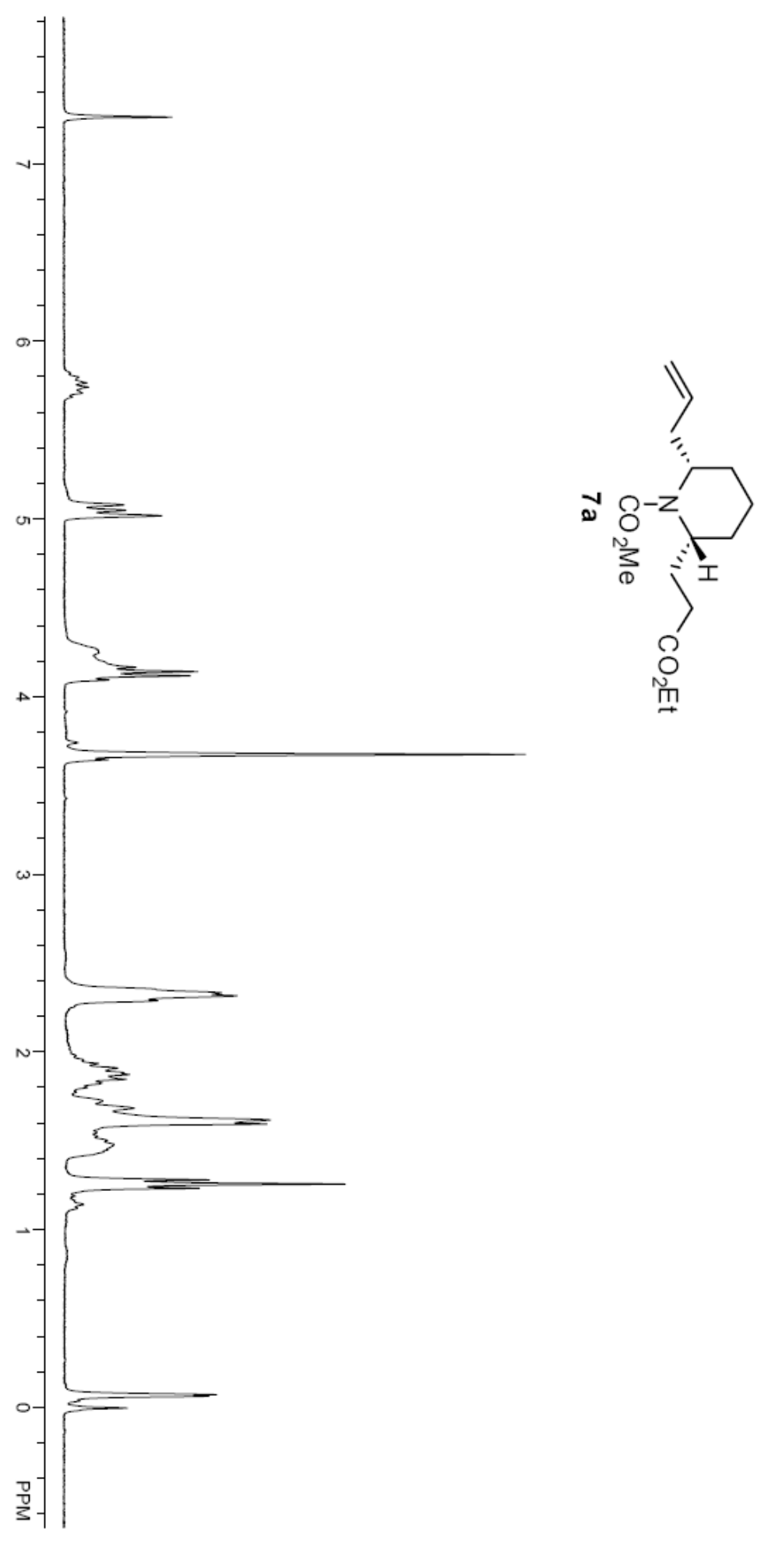



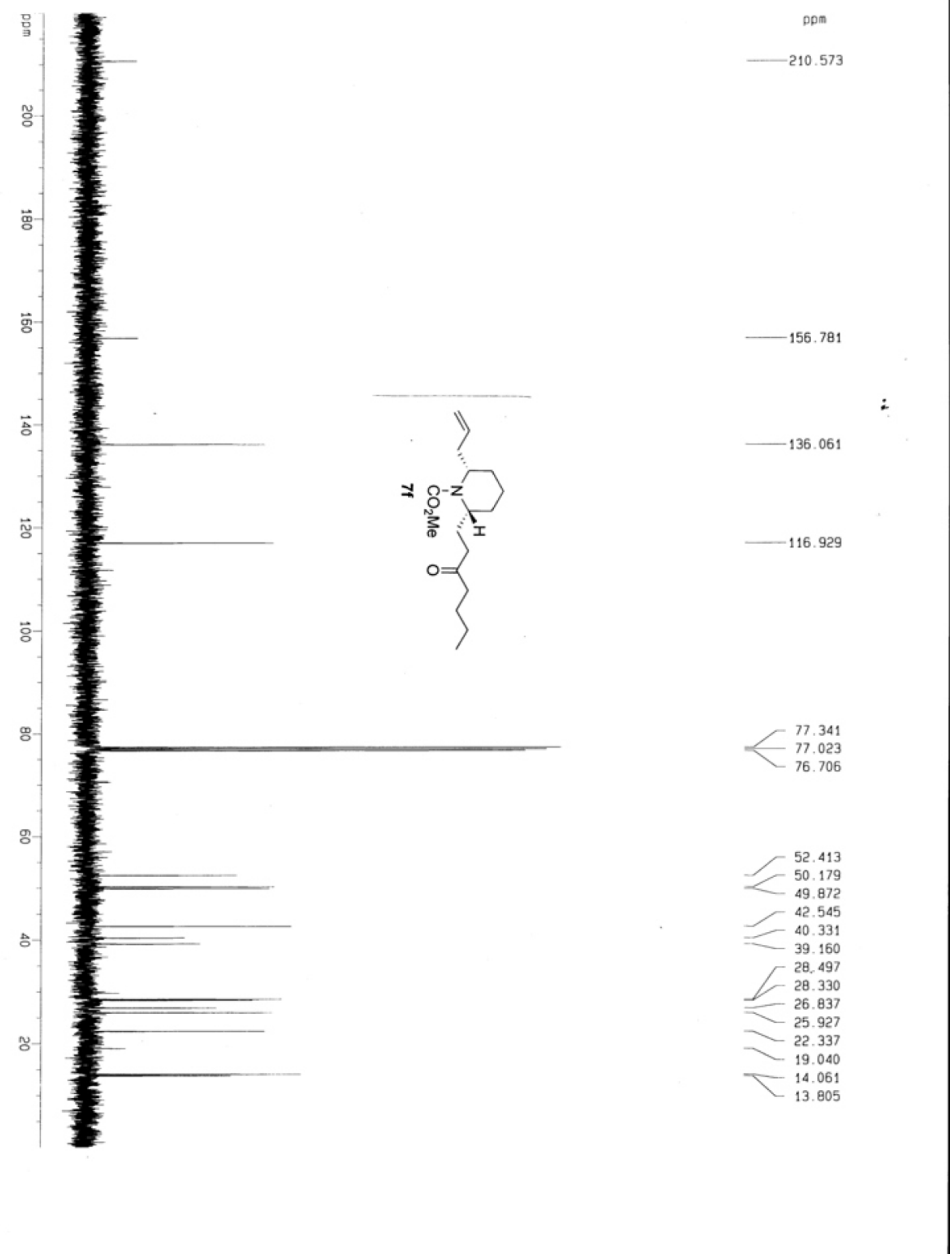


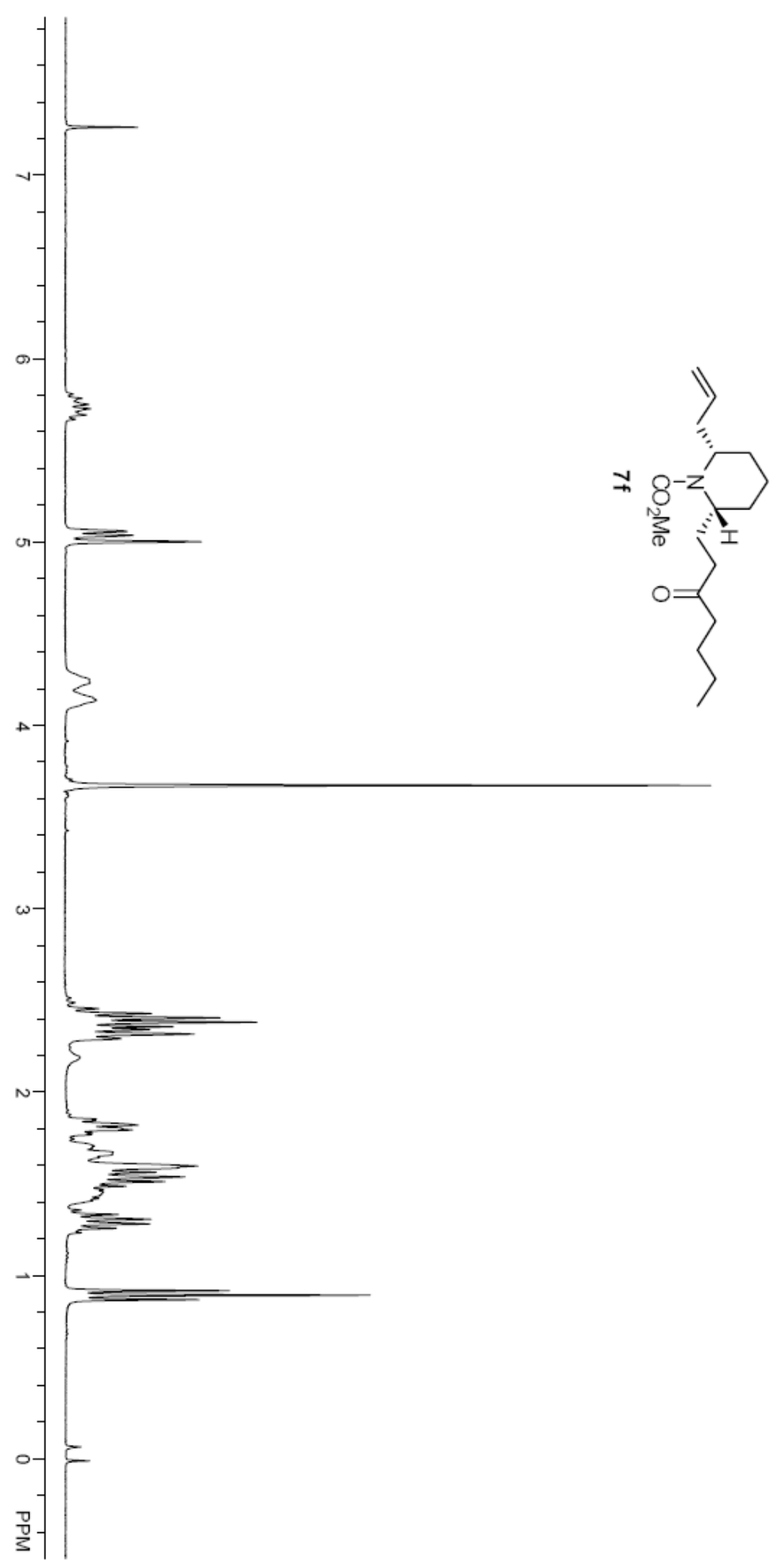




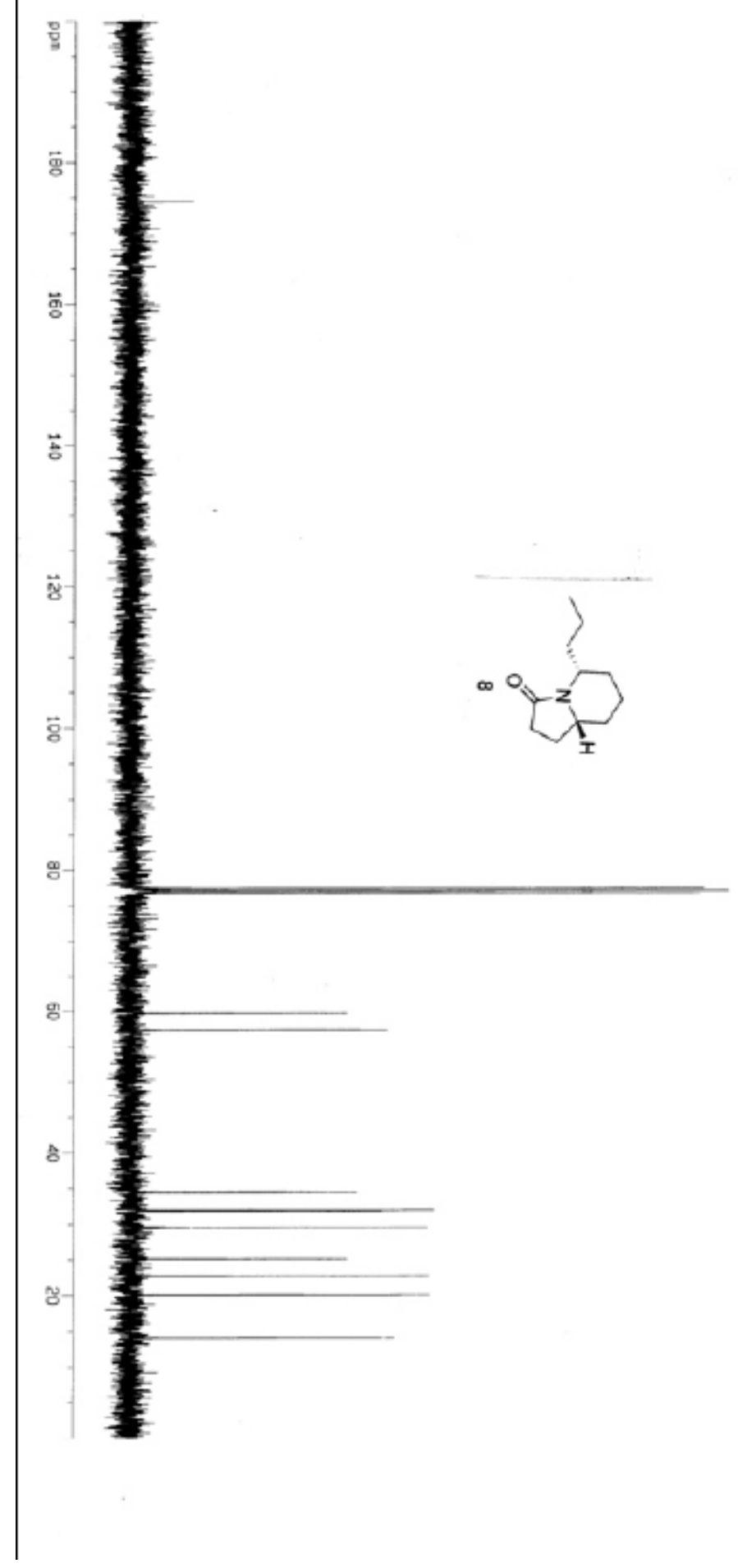

$p p m$

77.020
-76.702

- 59.678

57. 379

34.563

31.960

31.842

29.547

25.100

22.743

20.073

14.062 


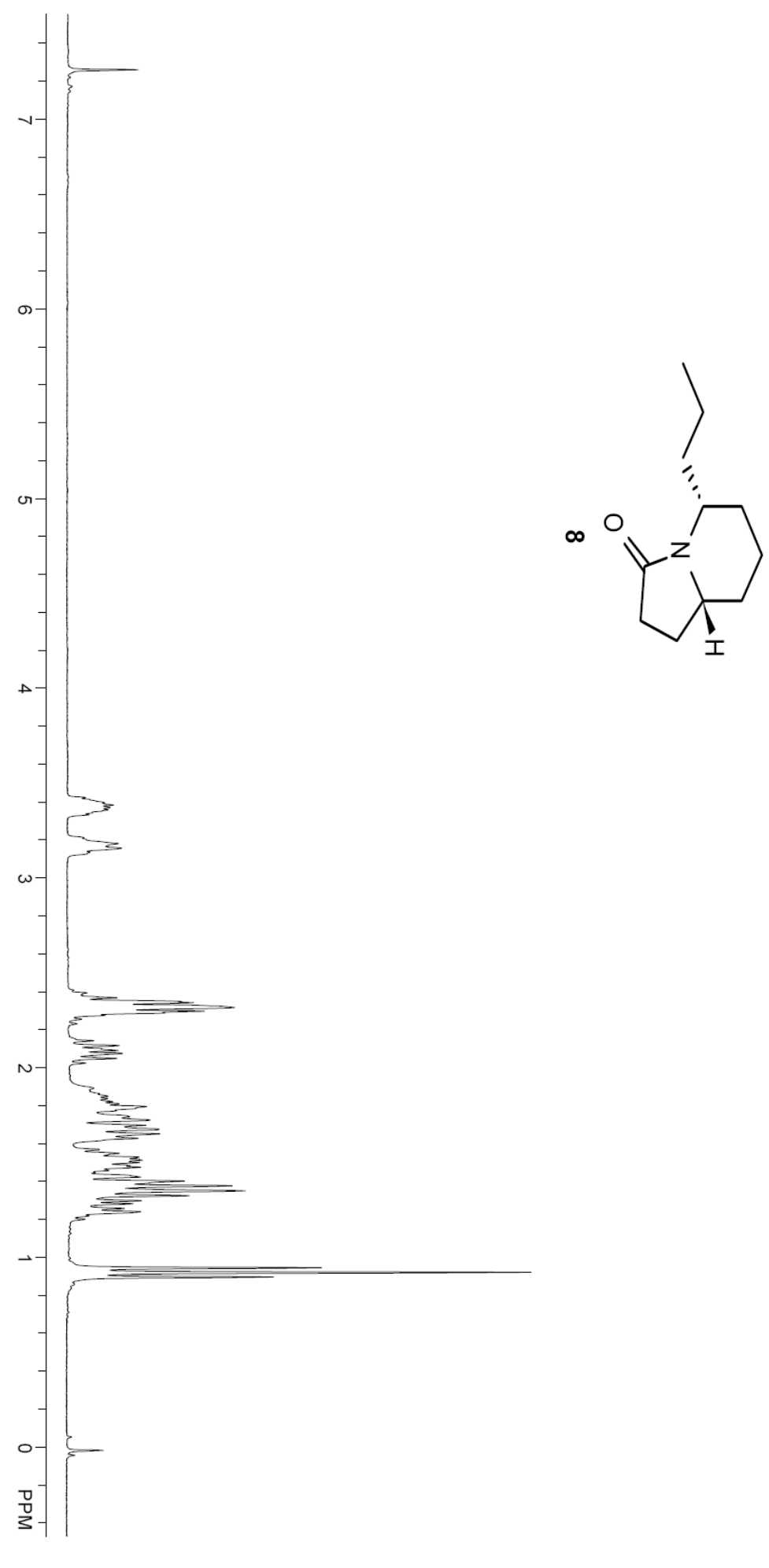



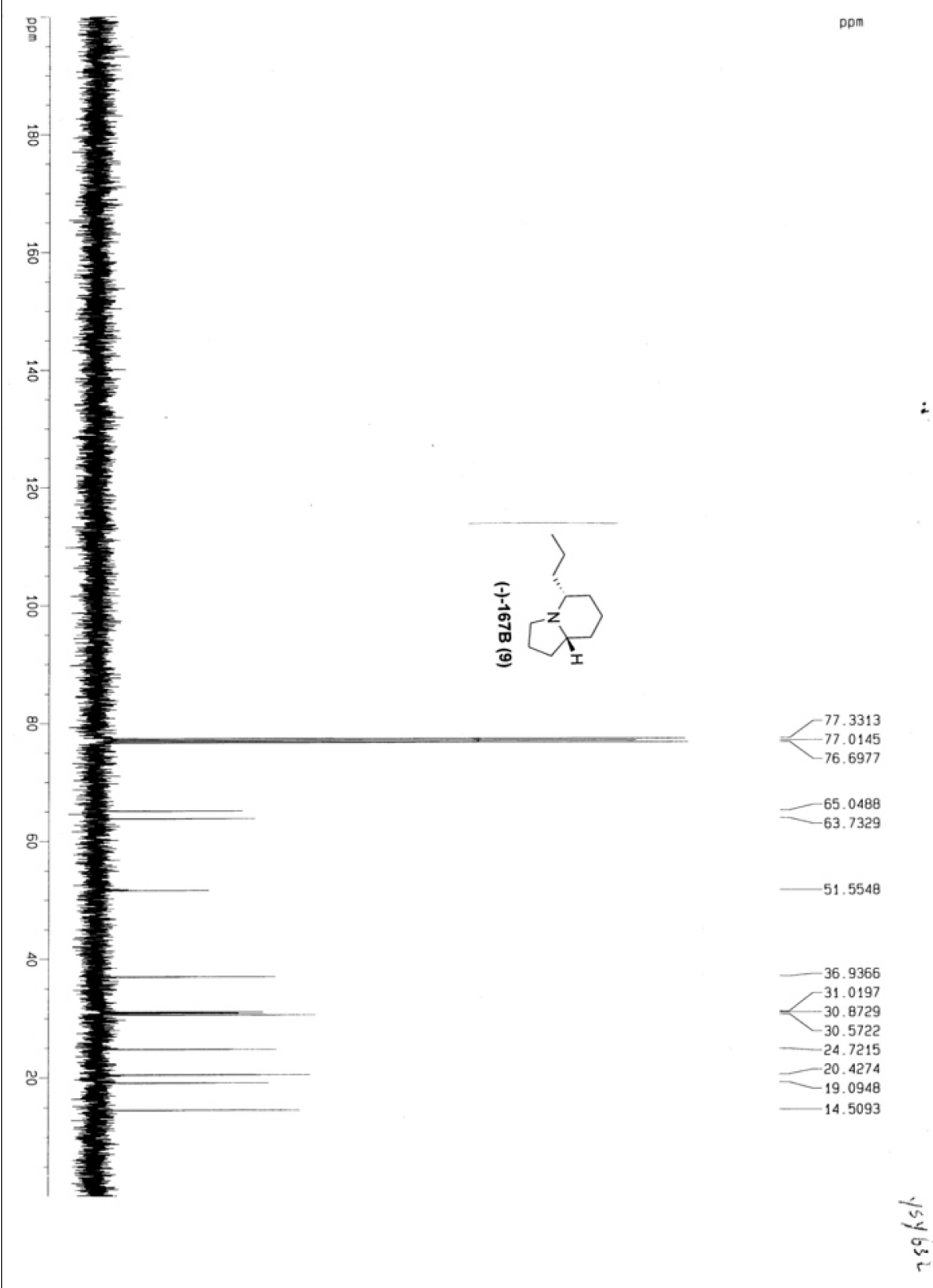


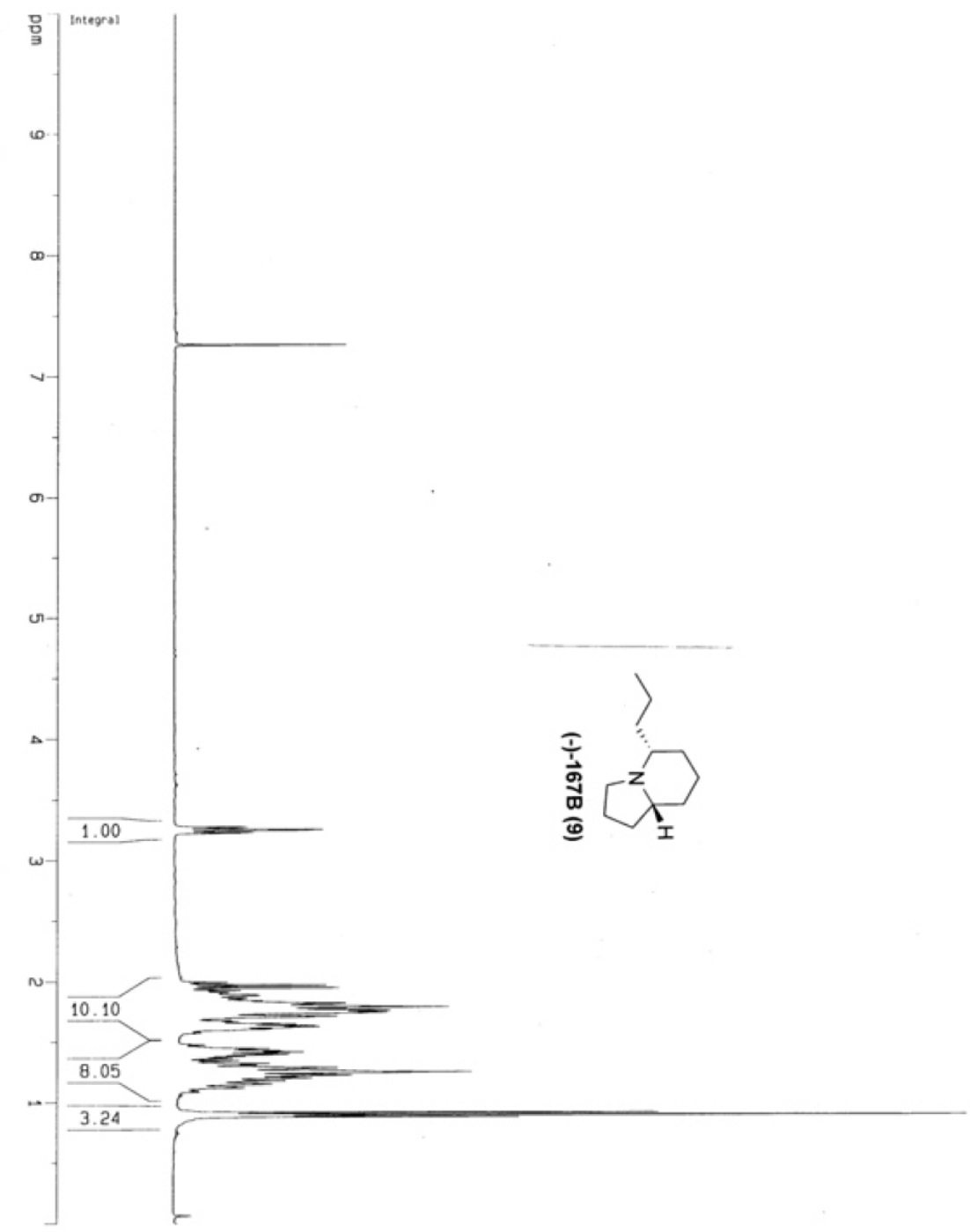

$\mathrm{Hz}$

$-2904.90$

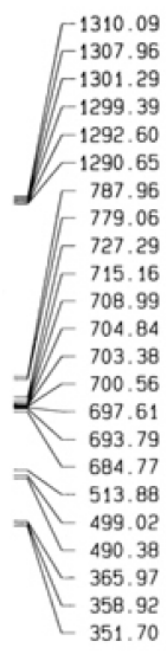

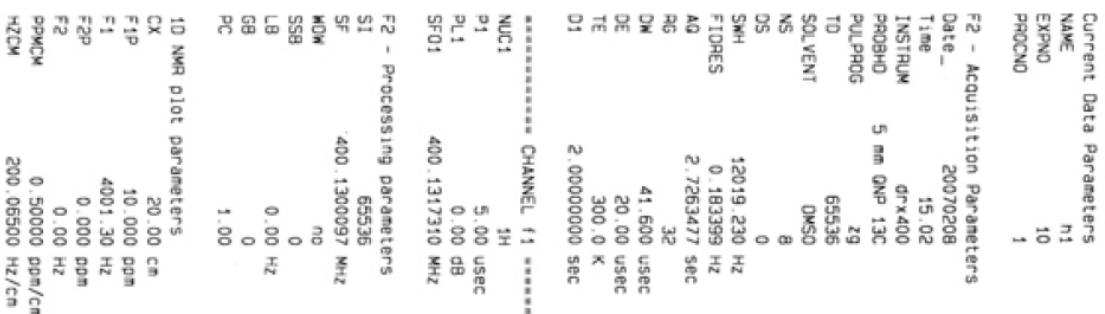




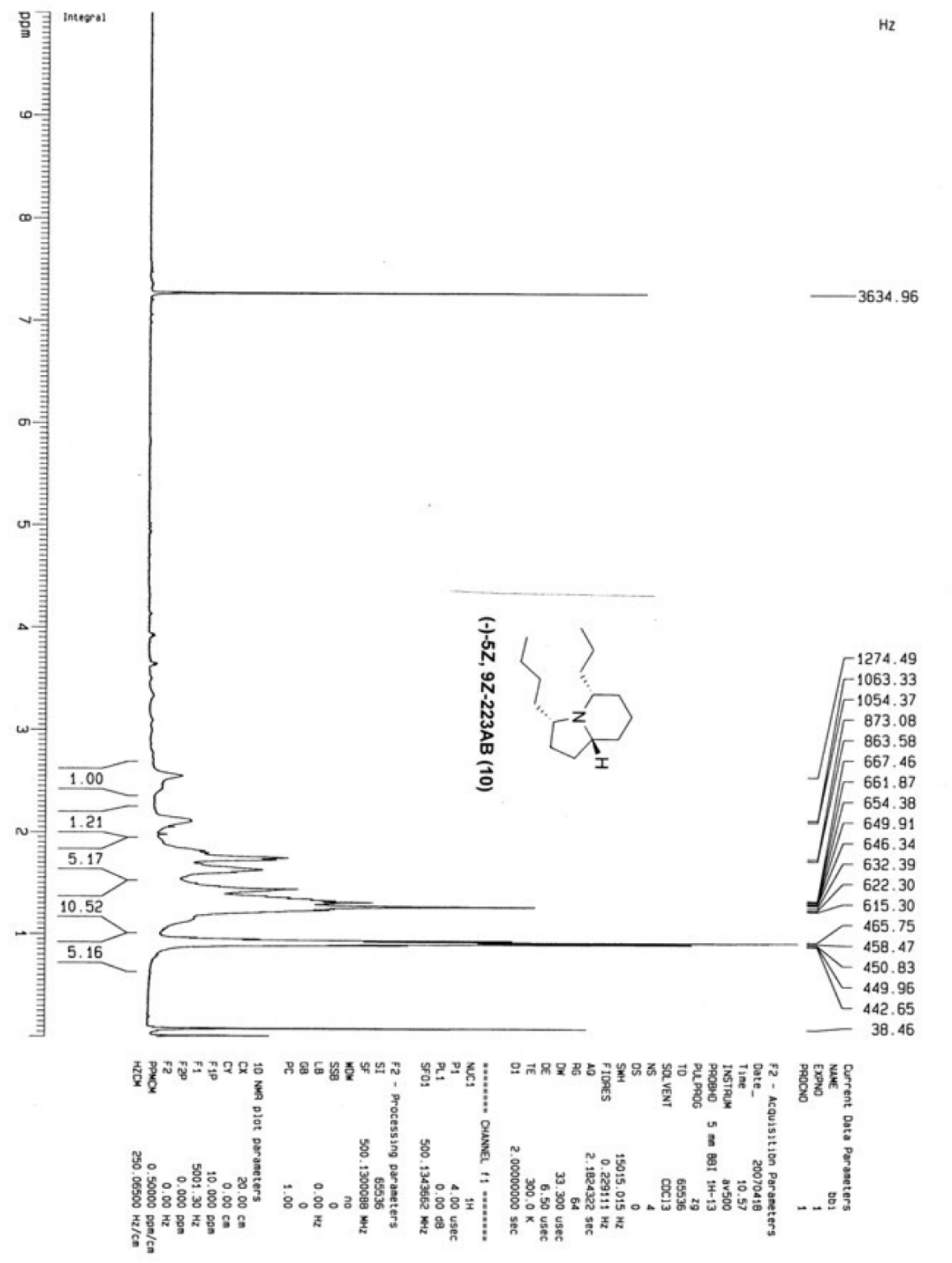

